\title{
Plant Natural Products for the Control of Aedes aegypti: The Main Vector of Important Arboviruses
}

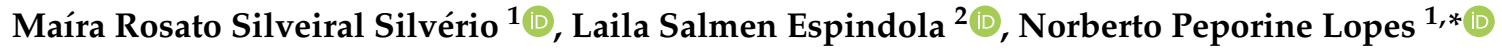 \\ and Paulo Cézar Vieira ${ }^{1, * \mathbb{D}}$ \\ 1 NPPNS, Departamento de Ciências BioMoleculares, Faculdade de Ciências Farmacêuticas de Ribeirão Preto, \\ Universidade de São Paulo, Ribeirão Preto 14040-903, São Paulo, Brazil; mairarosato@gmail.com \\ 2 Laboratório de Farmacognosia, Universidade de Brasília, Brasília 70910-900, Brazil; darvenne@unb.br \\ * Correspondence: npelopes@fcfrp.usp.br (N.P.L.); pcvieira@fcfrp.usp.br (P.C.V.); \\ Tel.: +55-16-3315-4707 (N.P.L.); +55-16-3315-4248 (P.C.V.)
}

Academic Editor: Roberto Fabiani

Received: 29 June 2020; Accepted: 28 July 2020; Published: 31 July 2020

\begin{abstract}
The mosquito species Aedes aegypti is one of the main vectors of arboviruses, including dengue, Zika and chikungunya. Considering the deficiency or absence of vaccines to prevent these diseases, vector control remains an important strategy. The use of plant natural product-based insecticides constitutes an alternative to chemical insecticides as they are degraded more easily and are less harmful to the environment, not to mention their lower toxicity to non-target insects. This review details plant species and their secondary metabolites that have demonstrated insecticidal properties (ovicidal, larvicidal, pupicidal, adulticidal, repellent and ovipositional effects) against the mosquito, together with their mechanisms of action. In particular, essential oils and some of their chemical constituents such as terpenoids and phenylpropanoids offer distinct advantages. Thiophenes, amides and alkaloids also possess high larvicidal and adulticidal activities, adding to the wealth of plant natural products with potential in vector control applications.
\end{abstract}

Keywords: Aedes aegypti; dengue; natural products; botanical species; essential oils; terpenes; phenylpropanoids; thiophenes; alkaloids; mechanisms of action

\section{Introduction}

The mosquito Aedes aegypti (Diptera: Culicidae) originated in Egypt and it is widely distributed in tropical and subtropical regions, including North America and Europe [1,2]. Ae. aegypti presents complete metamorphosis from immature egg, larva and pupa stages to the adult mosquito itself (Figure 1). The life cycle varies according to environmental temperature, food availability and quantity of larvae in the same breeding site. Under favorable conditions, after egg hatching, the mosquito transforms into the adult stage within 10 days, even though the eggs can be viable up to 450 days in the absence of water [3].

The female mosquito requires hematophagy for egg maturation. Viral transmission to humans occurs during this process if the mosquito is infected. The lifetime of a female mosquito is approximately 45 days [1]. Ae aegypti population control is considered the principal measure to combat arboviral diseases as this species is the primary vector of dengue, Zika, chikungunya and urban yellow fever $[4,5]$.

In 2012, dengue was considered the mosquito-borne disease of major importance in the world [5]. According to the World Health Organization, 390 million people are infected annually with the dengue virus, 96 million of which have clinical manifestations [6]. There are various symptoms, the first is usually high fever $\left(39-40{ }^{\circ} \mathrm{C}\right)$ with headache, prostration, arthralgia, anorexia, asthenia, nausea, among others. Some clinical aspects often depend on patient age. There is no specific treatment for dengue and the more complicated cases of the disease can cause hemorrhage, shock and even death [7]. 


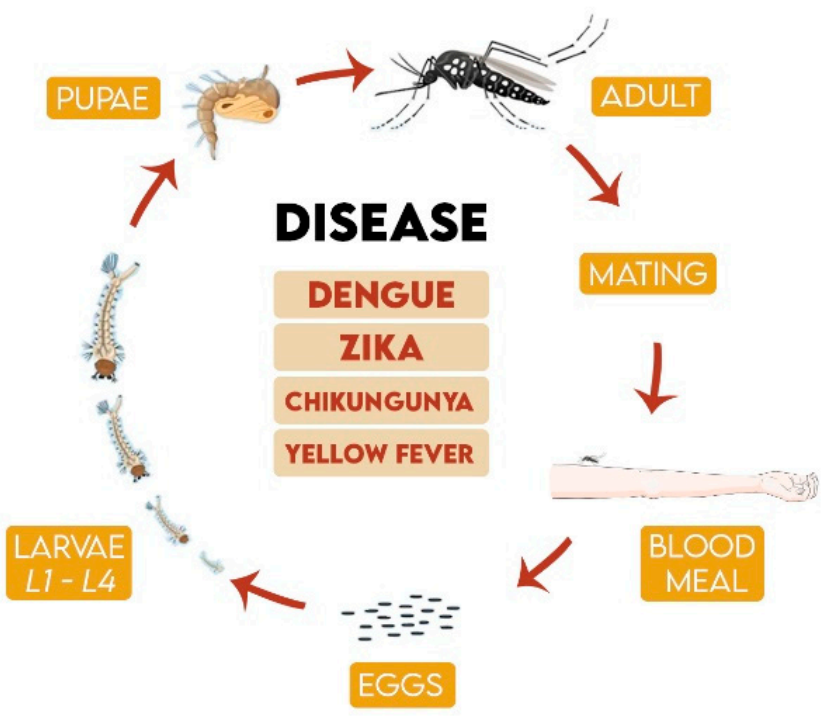

Figure 1. Aedes aegypti life cycle and the main arboviruses transmited by the female mosquito.

Ae. aegypti is considered the main Zika virus vector (Figure 1), but infection can also occur by sexual transmission or blood transfusion [8]. Symptoms are non-specific and self-limited, being easily confused with other arboviral diseases. Some important complications exist, such as microcephaly in fetuses and Guillan Barré syndrome. The virus has been reported in countries in Americas, Europe, Asia and the Pacific region [8]. Like dengue and Zika, chikungunya has no specific treatment. The disease emerged in the Americas in 2013, with about 1.7 million cases identified and 252 deaths reported by August 2015 [9].

In 2013, dengue generated a global cost of US $\$ 8.9$ billion, with around 58.4 million symptomatic cases (13.5 million fatalities) in the 141 countries and territories. The per capita costs of dengue were $\$ 70.1$ for hospital treatment, $\$ 51.1$ for outpatient treatment and $\$ 12.9$ for cases that did not reach the health system. According to this study, Brazil had an incidence of 751 to 1,000 cases per 100,000 people. The expenses were proportional to the incidence and ranged from $\$ 2.5$ to $\$ 5$ for each treated case [10].

A more recent study showed that in 2016 the Brazilian government spent around $\mathrm{R} \$ 805$ million (ca. 160 million US\$) to treat diseases caused by the Ae. aegypti mosquito, including direct medical expenses and indirect costs. In addition, about $\mathrm{R} \$ 1.5$ billion were destined to combat the vector, totaling R $\$ 2.3$ billion, that was $2 \%$ of the health budget for that year. More than 2 million cases of Ae. aegypti related diseases were verified. These numbers were underestimated as they did not include complications such as microcephaly and Guillain-Barré syndrome [11].

A study estimated that about $60 \%$ of the world population will be at risk of dengue in 2080 , which represents over 6.1 (4.7-6.9) billion people [12]. Considering that vector control is the main tool for controlling these expensive arboviruses, investment in techniques to combat the Ae. aegypti mosquito is growing [3]. Investments are particularly focused on techniques with minimal negative impacts on non-target animals and the environment $[4,13]$.

\section{Mosquito Control}

There are several techniques already used to combat mosquitoes, which act both in the immature phases (egg, larva and pupa) and in the adult [13]. Highly toxic synthetic insecticides such as organophosphates, pyrethroids and carbamates have been historically used to combat the mosquito, acting mainly on insect larvae [4]. More recently, insecticides with less toxicity which are less persistent in the environment have been developed, including neonicotinoids and oxadiazines [14]. However, these products are still widely used and harmful to living organisms and the environment, and the use of foogers and aerial applications of sintetic insecticides against adults, such as pyrethroids 
products, contributes to insect resistance problems [4]. Therefore, efforts must be made to ensure newly developed alternative insecticides are more eco-friendly.

Biological tools that control the adult stage are based on behavior, such as the Sterile Insect Technique (SIT), Incompatible Insect Technique (IIT) and Release of Insects carrying a Dominant Lethal gene (RIDL), which involve insect sterilization by chemical irradiation, natural bacteria which are pathogenic for mosquito (highly specific strains of Wolbachia) and genetic modifications to make sterile male mosquitoes, respectively $[15,16]$. The other technique applied against the adult stage is the use of entomopathogenic fungi, specifically in the orders Entomophthorales, Hypocreales and Pezizales due to their specificity, ability to manipulate and infectiveness to the host [17].

Ae. aegypti control using specific strains of Wolbachia bacteria is currently practiced in different locations around the world through the World Mosquito Program. This program involves the application of the bacteria to laboratory mosquitoes that are released into the local Ae. aegypti population during reproduction. The presence of bacteria in mosquitoes decreases the possibility of arbovirus transmission to people [18].

Biological control tools that act against the immature stages include the application of Bacillus thuringiensis in larvae habitats; products that prevent oviposition and/or inhibit growth and reproduction, including pheromones. There are also natural predators such as fish (especially of the genus Gambusia and Poecilia, family Poeciliidae) [19,20], copepods (including several species of the genus Mesocyclops) [21,22] and the "elephant mosquito" (genus Toxorhynchites) [23,24].

Finally, plant-based insecticides (ovicides, larvicides and pupicides) $[25,26]$ deserve a special mention due to the vast biodiversity of species found in the world, estimated to be approximately 400,000 terrestrial species [27]. Botanical insecticides can be plant extracts, essential oils and/or secondary metabolites $[4,14]$.

\section{Plant Natural Products to Control Mosquitoes}

The search for plant natural products to control Ae. aegypti dates back a number of years, with research published since the 1980s [28,29]. However, chemical insecticides are most commonly used, despite their enormous toxicity to non-target organisms, such as: (i) poisoning and death; (ii) cancer, by non-genotoxic mechanisms (immunosuppressants, cytotoxic) or by triggering the carcinogenic process in different ways; (iii) harmful effects on the nervous, renal, respiratory and reproductive systems and (iv) induction of oxidative stress [30].

In addition to toxicity, another concern is the increasing resistance of the mosquito vector to chemical insecticides. One example is the knockdown resistance $(k d r)$ mutation, in which resistance to pyrethroid insecticides occurs, whereby the target site is the sodium channel of the Ae. aegypti nervous system [31,32]. In Brazil, of the five insecticides approved by the Public Health Ministry and recommended by the $\mathrm{WHO}$ for adult mosquito control, four belong to the pyrethroid class together with one organophosphate (malathion). However, in 2011 a technical note was issued suspending the use of pyrethroids in Brazil to control Ae. aegypti [33].

The level of resistance is dependent on the insecticide concentration, frequency and duration of application [34]. The resistance mechanisms of mosquitoes may be associated with changes in the insect cuticle resulting in less insecticide absorption [35], changes in insect metabolism involving biotransformation enzymes [36,37] and modifications of the insecticide target site, usually by genetic mutations [38,39].

The main esterases involved in the resistance process are carboxylesterases and cholinesterases. Carboxylesterases are usually resistant to organophosphates, with this resistance relating to both a quantitative mechanism (overproduction of enzyme) and qualitative mechanism (mutations that cause alterations in enzymatic properties) [40]. In the case of cholinesterases, the resistance is mainly caused by gene mutation. The main insecticides resistant to the acetylcholinesterase target site are organophosphates and carbamates [41]. 
Another problem associated with chemical insecticides is the damage caused to the environment and living organisms by their degradation products, which may prove more toxic than the original product itself. Examples include the degradation products of temephos, whose effects have already been documented in aquatic environments [42], and malathion, together with its metabolites, in non-target organisms such as Daphnia magna (Cladocera: Daphniidae) [43].

Insecticides derived from plant natural products therefore offer a promising source of safer new products for mosquito control due to minimal residues from its natural degradation in both the field and in water, minimizing ecosystem disruption [44,45]. There is considerable research on insecticides of natural origin, especially those of microbial and plant origin, due to their innumerable secondary metabolites produced especially as a defense mechanism against natural predators [46]. It is estimated that there are more than 100,000 plant metabolites, with hundreds or more exhibiting some activity against insects [47].

Botanical insecticides are advantageous as they are generally environmentally safe, non-toxic to non-target organisms including homeothermic animals and their residues biodegradable $[25,26,30]$. The synergic mixture of the active compounds in extracts induce several mechanisms of action and result in less pest resistance [30,48].

The present review focuses on the more recent studies of botanical extracts and active compounds in applications against Ae. aegypti, from immature to adult stages, in addition to their main proposed mechanisms of action. The crude extracts are obtained using different extraction methods with organic solvents or water. Essential oils are obtained by steam distillation or hydrodistillation. The classes of active compounds include terpenes, alkaloids and amides, steroids, flavonoids, furanochromones, phenylpropanoids and phenol derivatives, lignans and neolignans, naphthoquinones, fatty acids and their derivatives. The type of insecticide activity (ovicide, larvicide, pupicide, adulticide) is reported as mortality and lethal concentration values $\left(\mathrm{LC}_{50}, \mathrm{LC}_{90}\right.$ and/or $\left.\mathrm{LC}_{99}\right)$, together with egg hatchability. The other activities tested are mosquito repellency, oviposition deterrence, growth regulation and the antifeedant effect.

\section{Essential Oils}

Essential oils deserve special attention as they have yields of 0.5 to $2.0 \%$ in the extraction process, contain a high concentratration of secondary metabolites and generally present potent activity due to the synergic effect of the constituents. An important advantage is, with few exceptions, their relatively low, or no, toxicity to mammals (Figure 2). Some pure compounds constituents of essential oils are moderately toxic to mammals ( $\mathrm{LD}_{50} 800-3000 \mathrm{mg} / \mathrm{kg}$ in rodents) while formulated products usually are low or non-toxic to mammals, birds and fish (LD 50 above $5000 \mathrm{mg} / \mathrm{kg}$ for rodents) [47,49].

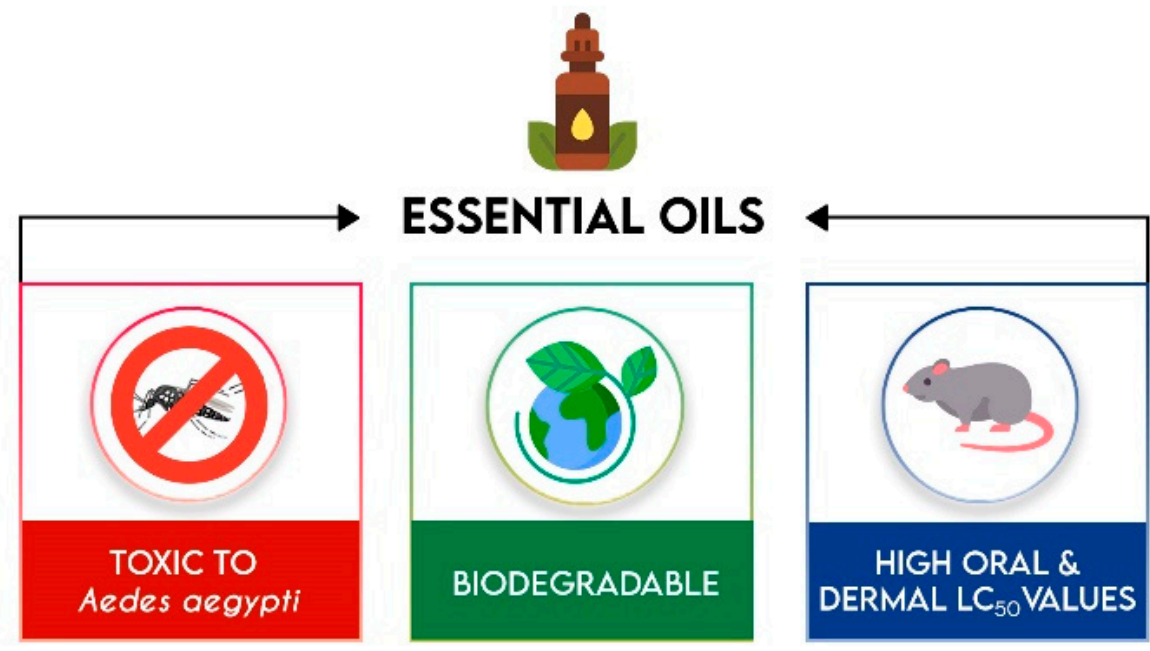

Figure 2. Essential oils to combat the Ae. aegypti mosquito: an ecologically safe alternative. 
These essential oils are mainly obtained from aromatic plants, of which there are more than 3000 species. Approximately $10 \%$ of these are already produced in large quantities for other uses, such as flavorings and fragrances, and are therefore readily available at reasonable prices [30]. Essential oils are composed of volatile compounds, which give an important advantage of non-persistence in the environment $[49,50]$.

It is important to note that the same volatility may be a disadvantage in terms of instability. However, this property can be overcome using pharmaceutical technology such as micro and nanoencapsulation [51,52]. Formulation development is therefore critical for essential oils to be used effectively and safely as pesticides. A number of studies have demonstrated that a suitable vehicle prolongs the insecticidal effect $[51,53,54]$.

Of the plant families affording essentials oils, those most tested against Ae. aegypti larvae were Myrtaceae, in particular Eucalyptus species, followed by Fabaceae, Asteraceae, Apiaceae and Lamiaceae. Asteraceae was the most important for the adulticide, repellent and oviposition effects.

Regarding larvicidal activity there is currently no value specified by the WHO to discriminate whether a compound or extract is active against insects. However, researchers usually consider that an $\mathrm{LC}_{50}<50 \mu \mathrm{g} / \mathrm{mL}$ is very active; an $\mathrm{LC}_{50} 50-100 \mu \mathrm{g} / \mathrm{mL}$ is active, and an $\mathrm{LC}_{50}>100 \mu \mathrm{g} / \mathrm{mL}$ is weak/inactive [55-57].

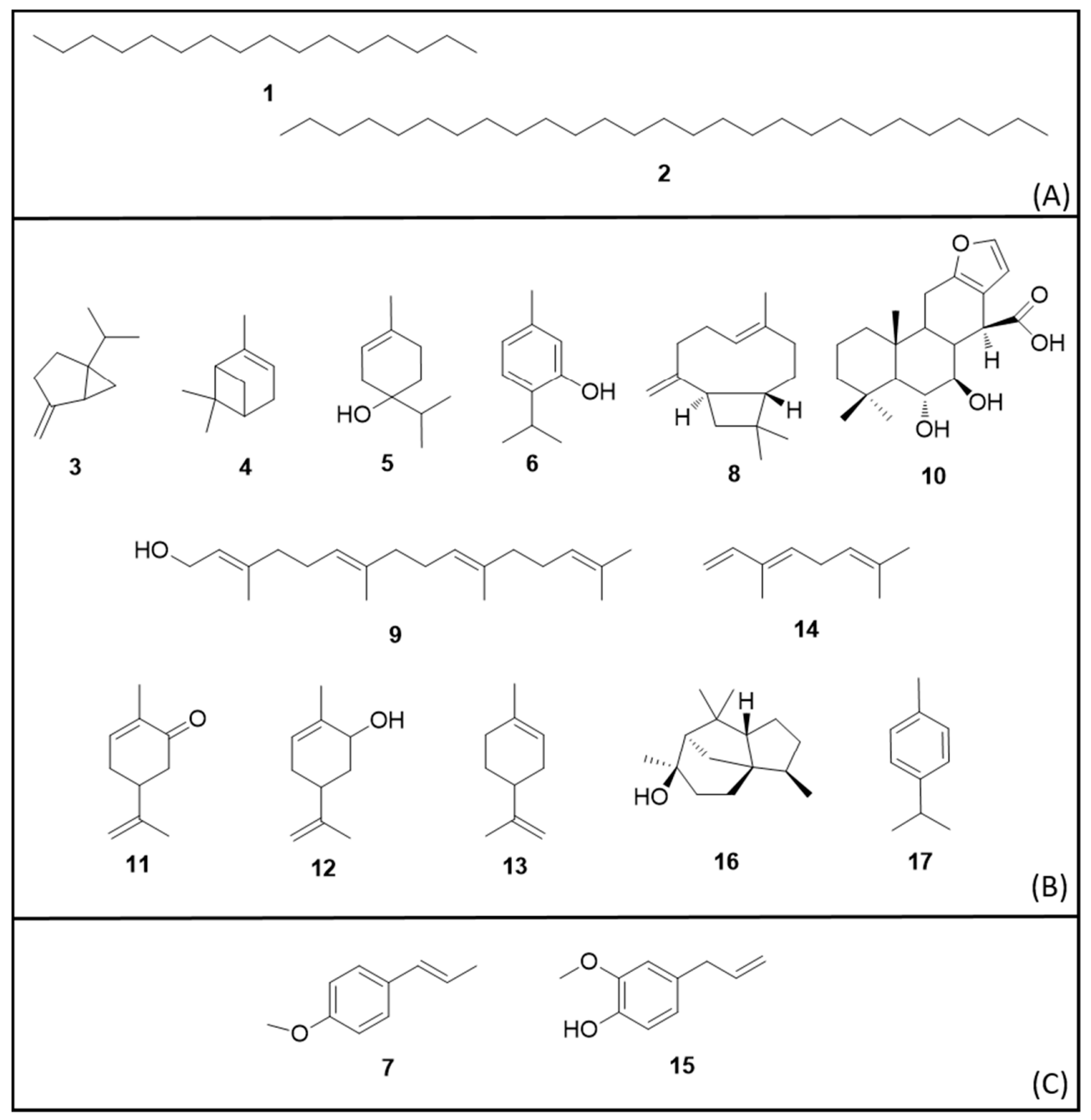

Figure 3. Secondary metabolites identified in essential oils with insecticidal activity against the Ae. aegypti. (A) Alkanes (B) Terpenes and (C) Phenylpropanoids. 
Considering this classification, this review highlights 11 species with 12 very active essential oils, 11 species with 14 active essential oils and 6 species with weak/no activity for 7 essential oils. Eight species do not have reported $\mathrm{LC}_{50}$ values and are not considered in this classification. However, these values can change significantly after formulation, as discussed in Section 8 "Limitations and/or Expectations of Plant Natural Product Insecticide Applications".

Some studies made the identification of secondary metabolites in essential oils evaluated for insectidal activities described abouve and its chemical structures are illustrated in Figure 3.

Tables 1 and 2 summarise the publications selected for this review and discussion of the essential oils active against the Ae. aeygpti mosquito. Table 1 describes larvicidal activities, while Table 2 details the adulticidal, repellent and oviposition activities.

The essential oil from Acacia nilotica (L.) Delile (Fabaceae) seeds had the highest insecticidal activity $\left(\mathrm{LC}_{50} 3.17 \mu \mathrm{g} / \mathrm{mL}\right)$. The major compounds were hexadecane (1) and heptacosane (2) [58]. This species has been investigated for several medicinal uses including spasmogenic and antiplasmodial activities of seed extracts [59].

Another species is Myristica fragans Houtt. (Myristicaceae), which is popularly known as nutmeg and is used as a flavoring. Essential oil from its seeds demonstrated high toxicity against Ae. aegypti, in both the $\mathrm{L} 3$ larval phase ( $\mathrm{LC}_{50} 28.2 \mu \mathrm{g} / \mathrm{mL}$ ) and the adult phase ( $\mathrm{LC}_{50} 18.5 \mu \mathrm{g} / \mathrm{mg}$ female). The major compounds identified were sabinene (3,52\%), $\alpha$-pinene (no stereochemistry defined, 4) $(13 \%)$ and terpinen-4-ol (5) (11\%). Regarding neurotoxic effects, this essential oil is non-toxic to humans as its $\mathrm{IC}_{50}$ values for human acetylcholinesterase and human butyrylcholinesterase are higher than $4000 \mu \mathrm{g} / \mathrm{mL}$ [54]. Nutmeg flower essential oil presented higher larvicidal activity (LC $5047.42 \mu \mathrm{g} / \mathrm{mL}$ ) than the ethanolic extract $\left(\mathrm{LC}_{50} 75.45 \mu \mathrm{g} / \mathrm{mL}\right.$ ). This result suggests that the constituents of the essential oil either exhibit higher larvicidal activity, or that the synergy between them favors the toxicity to the mosquito [60].

Eucalyptus species (Myrtaceae) leaf essential oils showed $\mathrm{LC}_{50}$ values in the range of 31.0-95.5 $\mu \mathrm{g} / \mathrm{mL}$ for the larvae stage and 100\% repellency for $1.5 \mathrm{~h}$. Eucalyptus camaldulensis Dehnh. had the highest larvicidal activity while Eucalyptus saligna Sm. displayed higher repellency than Eucalyptus nitens (H. Deane \& Maiden) Maiden [61-63].

Cinnamomum osmophloeum Kaneh. (Lauraceae) is commonly named pseudocinnamomum and the essential oil from leaves of different places demonstrating larvicidal activities of $\mathrm{LC}_{50} 36$ to $177 \mu \mathrm{g} / \mathrm{mL}$ [64]. Similar variation was observed in the larvicidal activity of different guava crops. The $\mathrm{LC}_{50}$ values of Psidium guajava L. (Myrtaceae) leaf essential oil ranged from 39.48 to $64.25 \mu \mathrm{g} / \mathrm{mL}$ [65].

Other edible plant essential oils that showed strong larvicidal activity were parsley [Petroselinum crispum (Mill.) A.W. Hill (Apiaceae)], fennel [Foeniculum vulgare Mill. (Apiaceae)], star anise [Illicium verum Hook. f. (Illiciaceae)], Piper sarmentosum Roxb. ex Hunt. (Piperaceae) and sucupira [Pterodon emarginatus Vogel (Fabaceae)] [54,60,66].

For parsley, the toxicity was evaluated for pyrethroid-susceptible and pyrethroid-resistant mosquitoes ( $\mathrm{LC}_{50} 40 \mu \mathrm{g} / \mathrm{mL}$ ) and the major metabolite was thymol (6). Similar toxicity was observed for fennel ( $\mathrm{LC}_{50} 44.84 \mu \mathrm{g} / \mathrm{mL}$ ), for star anise $\left(\mathrm{LC}_{50} 39.8 \mu \mathrm{g} / \mathrm{mL}\right.$ ) and for Piper sarmentosum (LC $\mathrm{L}_{50}$ $49.19 \mu \mathrm{g} / \mathrm{mL})[54,60]$. The major constituent of star anise essential oil was trans-anethole $(7,90 \%)[54]$. Furthermore, parsley and star anise essential oils demonstrated adulticidal activity with LC $506.01 \mu \mathrm{g} / \mathrm{mg}$ female for pyrethroid-susceptible, $\mathrm{LC}_{50} 6.15 \mu \mathrm{g} / \mathrm{mg}$ female for pyrethroid-resistant (Petroselinum crispum) and $\mathrm{LC}_{50} 10.3 \mu \mathrm{g} / \mathrm{mg}$ female for Illicium verum [54,60].

A sucupira (Pterodon emarginatus) fruit essential oil incorporated into a nanoemulsion to improve water solubility and increase product stability presented an $\mathrm{LC}_{50} 34.75 \mu \mathrm{g} / \mathrm{mL}$. The metabolites were identified as $\beta$-caryophyllene (8), geranylgeraniol (9) and $6 \alpha, 7 \beta$-dihydroxyvouacapan-17- $\beta$-oic acid (10). The toxicity for non-target organisms was tested in adult female Swiss albino mice [Mus musculus (Rodentia: Muridae)] with no behavioral effects, macroscopical changes or deaths reported [66]. 
Table 1. Larvicidal activity of essential oils against the Ae. aegypti mosquito.

\begin{tabular}{|c|c|c|c|c|c|c|c|c|c|c|}
\hline \multirow[b]{2}{*}{ Plant Species } & \multirow[b]{2}{*}{ Family } & \multirow[b]{2}{*}{ Country } & \multirow[b]{2}{*}{ Part Used } & \multirow[b]{2}{*}{ Larval Stage } & \multicolumn{4}{|c|}{ Mortality } & \multirow[b]{2}{*}{$\begin{array}{c}\text { Time of } \\
\text { Analysis (h) }\end{array}$} & \multirow[b]{2}{*}{ Reference } \\
\hline & & & & & \% Death & $\begin{array}{c}\text { Concentration } \\
(\mathrm{ppm})\end{array}$ & $\mathrm{LC}_{50}(\mathrm{ppm})$ & $\mathrm{LC}_{90}(\mathrm{ppm})$ & & \\
\hline Acacia nilótica (L.) Delile & Fabaceae & India & Seeds & L4 & ND & ND & 3.17 & 11.73 & 24 & [58] \\
\hline Alpinia purpurata (Viell.) K. Schum. & Zingiberaceae & Brazil & $\begin{array}{l}\text { Red flowers } \\
\text { Pink flowers }\end{array}$ & $\begin{array}{l}\mathrm{L} 4 \\
\mathrm{~L} 4\end{array}$ & $\begin{array}{l}\text { ND } \\
\text { ND }\end{array}$ & $\begin{array}{l}\text { ND } \\
\text { ND }\end{array}$ & $\begin{array}{l}80.70 \\
71.50\end{array}$ & ND & 24 & [67] \\
\hline Baccharis reticularia DC. & Asteraceae & Brazil & Leaves & L4 & ND & ND & 221.27 & 457.47 & 24 & [68] \\
\hline Bauhinia pulchella Benth. & Fabaceae & Brazil & Leaves & L3 & ND & ND & 105.90 & ND & 24 & [69] \\
\hline Bauhinia ungulata $\mathrm{L}$. & Fabaceae & Brazil & Leaves & L3 & ND & ND & 75.10 & ND & 24 & [69] \\
\hline Cinnamomum osmophloeum Kaneh. & Lauraceae & Taiwan & Leaves & $\mathrm{L} 4$ & ND & ND & 36.0 to 177.0 & 79.0 to 296.0 & 24 & [64] \\
\hline Croton rhamnifolioides Pax \& K. Hoffm. & Euphorbiaceae & Brazil & Leaves & $\mathrm{L} 4$ & ND & ND & $\begin{array}{c}89.0 \text { and } \\
122.30\end{array}$ & ND & 24 & {$[70]$} \\
\hline Cunninghamia konishii Hayata & Taxodiaceae & Taiwan & $\begin{array}{l}\text { Wood } \\
\text { Leaves }\end{array}$ & $\begin{array}{l}\text { L4 } \\
\text { L4 }\end{array}$ & $\begin{array}{l}\text { ND } \\
\text { ND }\end{array}$ & $\begin{array}{l}\text { ND } \\
\text { ND }\end{array}$ & $\begin{array}{l}85.70 \\
91.70\end{array}$ & $\begin{array}{l}171.40 \\
176.50\end{array}$ & 24 & {$[71]$} \\
\hline Curcuma longa $\mathrm{L}$. & Zingiberaceae & Thailand & Rhizome & $\mathrm{L} 4(\mathrm{p}-\mathrm{s})$ & ND & ND & 65.51 & 110.93 & 24 & {$[60]$} \\
\hline $\begin{array}{c}\text { Eucalyptus camaldulensis Dehnh. } \\
\text { Eucalyptus nitens (H. Deane \& Maiden) } \\
\text { Maiden }\end{array}$ & Myrtaceae & Argentina & Leaves & $\mathrm{L} 3 / \mathrm{L} 4$ & ND & ND & 52.83 & 71.80 & 24 & [62] \\
\hline Eucalyptus urophylla S.T. Blake & Myrtaceae & Taiwan & Leaves & L4 & ND & ND & 95.50 & 166.30 & 24 & [61] \\
\hline Ferula galbaniflua Boiss. \& Buhse & Apiaceae & Corea & Resin & L3 & 90 & 100.0 & ND & ND & 48 & [45] \\
\hline Foeniculum vulgare Mill. & Apiaceae & Thailand & Fruit & L4 (p-s) & ND & ND & 44.84 & 57.05 & 24 & [60] \\
\hline Hyssopus officinalis L. & Lamiaceae & Corea & Flowers & L3 & 95 & 100.0 & ND & ND & 48 & [45] \\
\hline Illicium verum Hook. f. & Illiciaceae & Brazil & Fruit & L3 & ND & ND & 39.80 & 53.0 & 24 & [54] \\
\hline Larix europea Lam. \& A. DC. & Pinaceae & Corea & Resin & L3 & 87 & 100.0 & ND & ND & 48 & [45] \\
\hline Limnophila aromatica (Lamk.) Merr. & Scrophulariaceae & Thailand & Whole plant & L4 (p-s) & ND & ND & 47.94 & 65.14 & 24 & [60] \\
\hline Mentha spicata $\mathrm{L}$. & Lamiaceae & India & Leaves & L3 & ND & ND & 56.08 & 110.28 & 24 & [72] \\
\hline Myristica fragrans Houtt. & Myristicaceae & $\begin{array}{c}\text { Thailand } \\
\text { Brazil }\end{array}$ & $\begin{array}{l}\text { Flowers } \\
\text { Seeds }\end{array}$ & $\begin{array}{l}\text { L4 (p-s) } \\
\text { L3 }\end{array}$ & $\begin{array}{l}\text { ND } \\
\text { ND }\end{array}$ & $\begin{array}{l}\text { ND } \\
\text { ND }\end{array}$ & $\begin{array}{l}47.42 \\
28.20\end{array}$ & $\begin{array}{l}69.28 \\
41.70\end{array}$ & $\begin{array}{l}24 \\
24\end{array}$ & [60] \\
\hline Myroxylon pereirae (Royle) Klotzsch & Fabaceae & Corea & Resin & L3 & $\begin{array}{c}97.5 \\
95\end{array}$ & $\begin{array}{c}100.0 \\
50.0\end{array}$ & $\begin{array}{l}\mathrm{ND} \\
\mathrm{ND}\end{array}$ & $\begin{array}{l}\text { ND } \\
\text { ND }\end{array}$ & 48 & {$[45]$} \\
\hline Pelargonium graveolens L'Hér. ex Aiton & Geraniaceae & Corea & Leaves & $\begin{array}{c}\text { L3 } \\
\text { L4 (p-s) }\end{array}$ & $\begin{array}{c}82 \\
\mathrm{ND}\end{array}$ & $\begin{array}{l}100.0 \\
\mathrm{ND}\end{array}$ & $\begin{array}{c}\mathrm{ND} \\
43.22\end{array}$ & $\begin{array}{c}\mathrm{ND} \\
66.60\end{array}$ & $\begin{array}{l}48 \\
24\end{array}$ & [45] \\
\hline Petroselinum crispum (Mill.) A.W. Hill & Apiaceae & Thailand & Fruit & $\begin{array}{l}\text { L4 (p-s 1) } \\
\text { L4 (p-s 2) }\end{array}$ & $\begin{array}{l}\text { ND } \\
\text { ND }\end{array}$ & $\begin{array}{l}\mathrm{ND} \\
\mathrm{ND}\end{array}$ & $\begin{array}{l}44.50 \\
44.03\end{array}$ & $\begin{array}{l}68.29 \\
67.71\end{array}$ & $\begin{array}{l}24 \\
24\end{array}$ & {$[60]$} \\
\hline Pimenta dioica (L.) Merr. & Myrtaceae & Brazil & Fruit & L3 & ND & ND & 104.40 & 137.30 & 24 & [54] \\
\hline Pinus sylvestris L. & Pinaceae & Nigeria & Needles & L4 & ND & ND & 100.39 & ND & 24 & [73] \\
\hline Piper aduncum $\mathrm{L}$. & Piperaceae & Brazil & Leaves & L3/L4 & $\begin{array}{l}\text { ND } \\
\text { ND }\end{array}$ & $\begin{array}{l}\text { ND } \\
\text { ND }\end{array}$ & $\begin{array}{l}289.90 \\
134.10\end{array}$ & $\begin{array}{l}654.90 \\
527.10\end{array}$ & $\begin{array}{l}24 \\
48\end{array}$ & [74] \\
\hline
\end{tabular}


Table 1. Cont.

\begin{tabular}{|c|c|c|c|c|c|c|c|c|c|c|}
\hline \multirow[b]{2}{*}{ Plant Species } & \multirow[b]{2}{*}{ Family } & \multirow[b]{2}{*}{ Country } & \multirow[b]{2}{*}{ Part Used } & \multirow[b]{2}{*}{ Larval Stage } & \multicolumn{4}{|c|}{ Mortality } & \multirow[b]{2}{*}{$\begin{array}{c}\text { Time of } \\
\text { Analysis (h) }\end{array}$} & \multirow[b]{2}{*}{ Reference } \\
\hline & & & & & \% Death & $\begin{array}{l}\text { Concentration } \\
(\mathrm{ppm})\end{array}$ & $\mathrm{LC}_{50}(\mathrm{ppm})$ & $\mathrm{LC}_{90}(\mathrm{ppm})$ & & \\
\hline Piper sarmentosum Roxb. ex Hunt. & Piperaceae & Thailand & $\begin{array}{l}\text { Stem and } \\
\text { Leaves }\end{array}$ & L4 (p-s) & ND & ND & 49.19 & 75.10 & 24 & [60] \\
\hline Pogostemon patchouli Pellet. & Lamiaceae & Corea & Whole plant & L3 & 97 & 100.0 & ND & ND & 48 & [45] \\
\hline Porophyllum ruderale (Jacq.) Cass. & Asteraceae & Brazil & $\begin{array}{l}\text { Flowers and } \\
\text { leaves }\end{array}$ & $\begin{array}{l}\text { L3 } \\
\text { L4 }\end{array}$ & $\begin{array}{l}\text { ND } \\
\text { ND }\end{array}$ & $\begin{array}{l}\text { ND } \\
\text { ND }\end{array}$ & $\begin{array}{l}60.90 \\
72.28\end{array}$ & $\begin{array}{l}132.48 \\
173.65\end{array}$ & $\begin{array}{l}24 \\
24\end{array}$ & [75] \\
\hline Psidium guajava $\mathrm{L}$. & Myrtaceae & Brazil & Leaves & L4 & ND & ND & $\begin{array}{l}39.48 \text { to } \\
64.25\end{array}$ & 57.34 to 86.0 & 24 & [65] \\
\hline Pterodon emarginatus Vogel & Fabaceae & Brazil & Fruit & L4 & ND & ND & 34.75 & ND & 48 & [66] \\
\hline Rosmarinus officinalis $\mathrm{L}$. & Lamiaceae & Brazil & Leaves & L4 & $\begin{array}{l}80 \\
90\end{array}$ & $\begin{array}{l}250.0 \\
250.0\end{array}$ & $\begin{array}{l}\text { ND } \\
\text { ND }\end{array}$ & $\begin{array}{l}\text { ND } \\
\text { ND }\end{array}$ & $\begin{array}{l}24 \\
48\end{array}$ & [76] \\
\hline Sphaeranthus indicus L. & Asteraceae & India & Leaves & L4 & ND & $\mathrm{ND}$ & 140.0 & 350.0 & 24 & [77] \\
\hline $\begin{array}{c}\text { Syzygium aromaticum (L.) Merr. \& L.M. } \\
\text { Perry }\end{array}$ & Myrtaceae & Nigeria & Bud & L4 & ND & ND & 92.56 & $\mathrm{ND}$ & 24 & [73] \\
\hline $\begin{array}{l}\text { Tanacetum argenteum (Lam.) Willd. } \\
\text { subsp. argenteum (Lam.) }\end{array}$ & Asteraceae & Turkey & Aerial parts & L1 & ND & ND & 93.30 & 241.70 & 24 & [78] \\
\hline Trachyspermum ammi (L.) Sprague & Apiaceae & Corea & Seeds & L3 & $\begin{array}{c}100 \\
80\end{array}$ & $\begin{array}{c}100.0 \\
50.0\end{array}$ & $\begin{array}{l}\text { ND } \\
\text { ND }\end{array}$ & $\begin{array}{l}\text { ND } \\
\text { ND }\end{array}$ & 48 & [45] \\
\hline
\end{tabular}

$\mathrm{LC}_{50}$ lethal concentration required to kill $50 \%$ of the larval population, $\mathrm{LC}_{90}$ lethal concentration required to kill $90 \%$ of the larval population, ND not described, $\mathrm{p}$-s pyrethroid-susceptible. 
Table 2. Adulticidal, repellent and oviposition activities of essential oils against the Ae. aegypti mosquito.

\begin{tabular}{|c|c|c|c|c|c|c|c|}
\hline Plant Species & Family & Country & Part Used & Activity & Results & $\begin{array}{c}\text { Time of } \\
\text { Analysis (h) }\end{array}$ & Reference \\
\hline $\begin{array}{c}\text { Acantholippia seriphioides (A. Gray) } \\
\text { Moldenke }\end{array}$ & Verbenaceae & Argentina & ND & Repellent & $100 \%$ of repellency at $50 \%$ & 1.2 & [63] \\
\hline Aloysia citriodora Palau & Verbenaceae & Argentina & ND & Repellent & $100 \%$ of repellency at $12.5 \%$ & 1.5 & [63] \\
\hline Alninia nurnurata (Viell) K Schum & Zinoiberaceae & Brazil & Red flowers & Oviposition & Oviposition disruptive effect & ND & [67] \\
\hline Alpınıa purpurata (Viell.) K. Schum. & Zingiberaceae & Brazil & Pink flowers & Oviposition & Oviposition disruptive effect & ND & [67] \\
\hline Baccharis spartioides (Hook. \& Arn.) Remy & Asteraceae & Argentina & ND & Repellent & $100 \%$ of repellency at $12.5 \%$ & 1.5 & [63] \\
\hline Croton rhamnifolioides Pax \& K. Hoffm. & Euphorbiaceae & Brazil & Leaves & Oviposition & Only $30 \%$ of oviposition at $100.0 \mu \mathrm{g} / \mathrm{mL}$ & 16 & [70] \\
\hline $\begin{array}{l}\text { Eucalyptus nitens (H. Deane \& Maiden) } \\
\text { Maiden }\end{array}$ & Myrtaceae & Argentina & Leaves & Repellent & $100 \%$ pure repellency & 1.5 & [62] \\
\hline Eucalyptus saligna $\mathrm{Sm}$. & Myrtaceae & Argentina & ND & Repellent & $100 \%$ of repellency at $50 \%$ & 1.5 & [63] \\
\hline Illicium verum Hook. f. & Illiciaceae & Brazil & Fruit & Adulticide & $\begin{array}{l}\mathrm{LC}_{50} 10.30 \mu \mathrm{g} / \mathrm{mg} \text { female } \\
\mathrm{LC}_{90} 17.50 \mu \mathrm{g} / \mathrm{mg} \text { female }\end{array}$ & 24 & [54] \\
\hline Minthostachys mollis Griseb & Lamiaceae & Argentina & ND & Repellent & $100 \%$ of repellency at $50 \%$ & 1.0 & [63] \\
\hline Myristica fragrans Houtt. & Myristicaceae & Brazil & Seeds & Adulticide & $\begin{array}{l}\mathrm{LC}_{50} 18.50 \mu \mathrm{g} / \mathrm{mg} \text { female } \\
\mathrm{LC}_{90} 31.90 \mu \mathrm{g} / \mathrm{mg} \text { female }\end{array}$ & 24 & [54] \\
\hline Petroselinum crispum (Mill.) A.W. Hill & Apiaceae & Thailand & Fruit & $\begin{array}{l}\text { Adulticide (p-s) } \\
\text { Adulticide (p-r) }\end{array}$ & $\begin{array}{l}\mathrm{LC}_{50} 6.01 \mu \mathrm{g} / \mathrm{mg} \text { female } \\
\mathrm{LC}_{90} 9.39 \mu \mathrm{g} / \mathrm{mg} \text { female } \\
\text { LC }_{50} 6.15 \mu \mathrm{g} / \mathrm{mg} \text { female } \\
\text { LC }_{90} 9.82 \mu \mathrm{g} / \mathrm{mg} \text { female }\end{array}$ & 24 & [60] \\
\hline Pimenta dioica (L.) Merr. & Myrtaceae & Brazil & Fruit & Adulticide & $\begin{array}{l}\mathrm{LC}_{50} 16.6 \mu \mathrm{g} / \mathrm{mg} \text { female } \\
\mathrm{LC}_{90} 31.4 \mu \mathrm{g} / \mathrm{mg} \text { female }\end{array}$ & 24 & [54] \\
\hline Pluchea carolinensis (Jack.) G. & Asteraceae & Martinica & $\begin{array}{l}\text { Leaves and } \\
\text { flowers }\end{array}$ & $\begin{array}{l}\text { Repellent } \\
\text { Irritant }\end{array}$ & $\begin{array}{c}36.6 \% \text { of repellency at } 1.0 \% \\
66.2 \% \text { of irritation at } 0.1 \%\end{array}$ & $\begin{array}{l}\text { ND } \\
\text { ND }\end{array}$ & $\begin{array}{l}{[79]} \\
{[79]}\end{array}$ \\
\hline Rosmarinus officinalis $\mathrm{L}$. & Lamiaceae & Argentina & ND & Repellent & $100 \%$ of repellency at $50 \%$ & 1.5 & [63] \\
\hline Sphaeranthus indicus L. & Asteraceae & India & Leaves & Repellent & $100 \%$ of repellency at $200 \mathrm{ppm}$ & 3.5 & [77] \\
\hline Tagetes minuta $\mathrm{L}$. & Asteraceae & Argentina & ND & $\begin{array}{c}\text { Adulticide } \\
\text { Repellent }\end{array}$ & $\begin{array}{l}100 \% \text { of mortality at } 800 \mathrm{ppm} \\
100 \% \text { of repellency at } 25 \%\end{array}$ & $\begin{array}{l}24 \\
1.5\end{array}$ & {$[77]$} \\
\hline
\end{tabular}

$\mathrm{LC}_{50}$ lethal concentration required to kill $50 \%$ of the mosquito population, $\mathrm{LC}_{90}$ lethal concentration required to kill $90 \%$ of the mosquito population, ND not described, p-s pyrethroid-susceptible, p-r pyrethroid-resistant. 
The essential oil of spearmint [Mentha spicata L. (Lamiaceae)] leaves also showed larvicidal activity $\left(\mathrm{LC}_{50} 56.08 \mu \mathrm{g} / \mathrm{mL}\right)$. The main constituents were carvone (11) $(48.6 \%)$, cis-carveol (12, 21.3\%) and limonene $(\mathbf{1 3}, \mathbf{1 1 . 3} \%)$ [72]. Another spice with larvicidal acitivity was turmeric [Curcuma longa L. (Zingiberaceae)] whose rhizome essential oil demonstrated LC $_{50} 65.51 \mu \mathrm{g} / \mathrm{mL}$ [62]. Similarly, Porophyllum ruderale (Jacq.) Cass. (Asteraceae) leaves essential oil, a herb used for seasoning food, showed $\mathrm{LC}_{50} 60.9 \mu \mathrm{g} / \mathrm{mL}$ for L3 larvae and $\mathrm{LC}_{50} 72.3 \mu \mathrm{g} / \mathrm{mL}$ for L4. The main metabolite identified was $\beta$-ocimene $(\mathbf{1 4}, 94 \%)$ [75].

Two other species exhibited very similar $\mathrm{LC}_{50}$ values (ca. $93 \mu \mathrm{g} / \mathrm{mL}$ ): clove [Syzygium aromaticum (L.) Merr. \& L.M. Perry (Myrtaceae)] against L4 larvae and Tanacetum argenteum (Lam.) Willd. subsp. argenteum (Lam.) (Asteraceae) against L1 [73,78]. The major constituent of clove bud essential oil was eugenol $15(80 \%)$ [73]. The clove bud alcoholic extract is popularly used as a repellent.

Cheng et al. (2013) demonstrated that essential oils of different parts of Cunninghamia konishii Hayata (Taxodiaceae) were toxic to L4 larvae (wood LC $5085.7 \mu \mathrm{g} / \mathrm{mL}$ ) and (leaves LC $5091.7 \mu \mathrm{g} / \mathrm{mL}$ ). The ethanolic extracts were inactive: wood $\left(\mathrm{LC}_{50} 240 \mu \mathrm{g} / \mathrm{mL}\right)$ and leaves $\left(\mathrm{LC}_{50}>400 \mu \mathrm{g} / \mathrm{mL}\right)$. The essential oil major compounds were: cedrol (16,53.0\%) and $\alpha$-pinene $(4,25.6 \%$, wood), and $\alpha$-pinene $(4,35.9 \%)$ and $p$-cymene $(\mathbf{1 7}, 16.7 \%$, leaves $)$ [71]. The results suggest that compound synergy is important for essential oil larvicidal activity as isolated compounds showed lower toxicity, with the exception of p-cymene [71].

Alpinia purpurata (Viell.) K. Schum. (Zingiberaceae) and Croton rhamnifolioides Pax \& K. Hoffm. (Euphorbiaceae) demonstrated toxicity against L4 larvae and oviposition deterrent effect. The LC 50 of A. purpurata essential oil was $71.5 \mu \mathrm{g} / \mathrm{mL}$ (pink flowers) and $80.7 \mu \mathrm{g} / \mathrm{mL}$ (red flowers) [67], while the $\mathrm{LC}_{50}$ for C. rhamnifolioides was $89.0 \mu \mathrm{g} / \mathrm{mL}$ (fresh leaves) and $122.3 \mu \mathrm{g} / \mathrm{mL}$ (stored leaves) [70].

Other species, such as Baccharis reticularia DC. (Asteraceae); Piper aduncum L. (Piperaceae) and Pinus sylvestris L. (Pinaceae) showed weak larvicidal activities (LC 50 100.4-290.0 $\mu \mathrm{g} / \mathrm{mL})$ [68,73,74]. Bauhinia pulchella Benth. (Fabaceae) displayed weak activity $(105.9 \mu \mathrm{g} / \mathrm{mL})$ whereas Bauhinia ungulata L. (Fabaceae) was active (75.1 $\mu \mathrm{g} / \mathrm{mL})$ [69] and Pimenta dioica (L.) Merr. (Myrtaceae) was investigated for both larvicidal (weak activity, LC $_{50} 104.4 \mu \mathrm{g} / \mathrm{mL}$ ) [54] and adulticidal action (very active, $\left.\mathrm{LC}_{50} 16.6 \mu \mathrm{g} / \mathrm{mL}\right)[24]$.

The essential oil of Sphaeranthus indicus L. (Asteraceae) leaves showed poor activity: larvae (LC $50140 \mu \mathrm{g} / \mathrm{mL})$, adult $(800 \mu \mathrm{g} / \mathrm{mL}$ for $100 \%$ mortality) and repellent $(200 \mu \mathrm{g} / \mathrm{mL})$. This essential oil showed low toxicity $(1500 \mu \mathrm{g} / \mathrm{mL})$ to a non-target aquatic predator [Toxorhynchites splendens (Diptera: Culicidae)] [77]. Similarly, the essential oil of rosemary [Rosmarinus officinalis L. (Lamiaceae)] was poor active when incorporated into a nanoemulsion and tested for larvicidal activity. The mortality of L3 larvae at $250 \mu \mathrm{g} / \mathrm{mL}$ of product was $80 \%$ at $24 \mathrm{~h}$ and $90 \%$ at $48 \mathrm{~h}$ [76].

Seo et al. (2012) investigated the activity of 7 plant species essential oils against L3 larvae at $50 \mu \mathrm{g} / \mathrm{mL}$ and $100 \mu \mathrm{g} / \mathrm{mL}$. Mortality ranged from 80 to $100 \%$ after $48 \mathrm{~h}$. The species tested were Ferula galbaniflua Boiss. \& Buhse and Trachyspermum ammi (L.) Sprague (Apiaceae); Hyssopus officinalis L. and Pogostemon patchouli Pellet. (Lamiaceae); Larix europea Lam. \& A. DC. (Pinaceae); Myroxylon pereirae (Royle) Klotzsch (Fabaceae), and Pelargonium graveolens L'Hér. ex Aiton (Geraniaceae) [45]. Additional $\mathrm{LC}_{50}$ data is required to determine the degree of activity against Ae. aegypti larvae.

Repellent activity was reported for essentials oils of Acantholippia seriphioides (A. Gray) Moldenke (Verbenaceae) and Aloysia citriodora Palau (Verbenaceae); Baccharis spartioides (Hook. \& Arn.) Remy (Asteraceae) and Tagetes minuta L. (Asteraceae), and Minthostachys mollis Griseb and Rosmarinus officinalis. All of the aforementioned demonstrated 100\% repellency (12.5-50\%) [63]. In another study, Pluchea carolinensis (Jack.) G. (Asteraceae) demonstrated repellency (36.6\%) at 1.0\% concentration and irritation $(62.2 \%)$ at $0.1 \%$ concentration [79].

The results listed and discussed in this section clearly suggest that essential oils present a promising alternative to develop an effective natural and potentially more eco-friendly insecticide for the control of Ae. aegypti, especially during the larval phase. The challenges for these materials are to improve 
solubility in water and prolong the insecticidal effect. It is also important to understand the synergism and/or antagonism of their constituents, together with the optimum ratio.

\section{Organic/Aqueous Extracts}

Concerning organic/aqueous extracts, the plant families with the highest number of species tested against Ae. aegypti larvae were Fabaceae, Asteraceae, Piperaceae and Euphorbiaceae. Similarly, as described by Isman (2015), India was the country with the most publications in this field, followed by Brazil [14].

Of the 20 plant species, at least one organic/aqueous extract showed high larvicidal activity $\left(\mathrm{LC}_{50}<50 \mu \mathrm{g} / \mathrm{mL}\right) ; 12$ were active $\left(\mathrm{LC}_{50} 50-100 \mu \mathrm{g} / \mathrm{mL}\right.$ ) and 26 had weak activity $\left(\mathrm{LC}_{50}>100 \mu \mathrm{g} / \mathrm{mL}\right)$. Nevertheless, these values can change significantly after formulation in a similar way to essential oils, as described in Section 8 "Limitations and/or Expectations of Plant Natural Product Insecticidal Applications".

Figure 4 details the chemical structures of the secondary metabolites identified in the organic extracts.

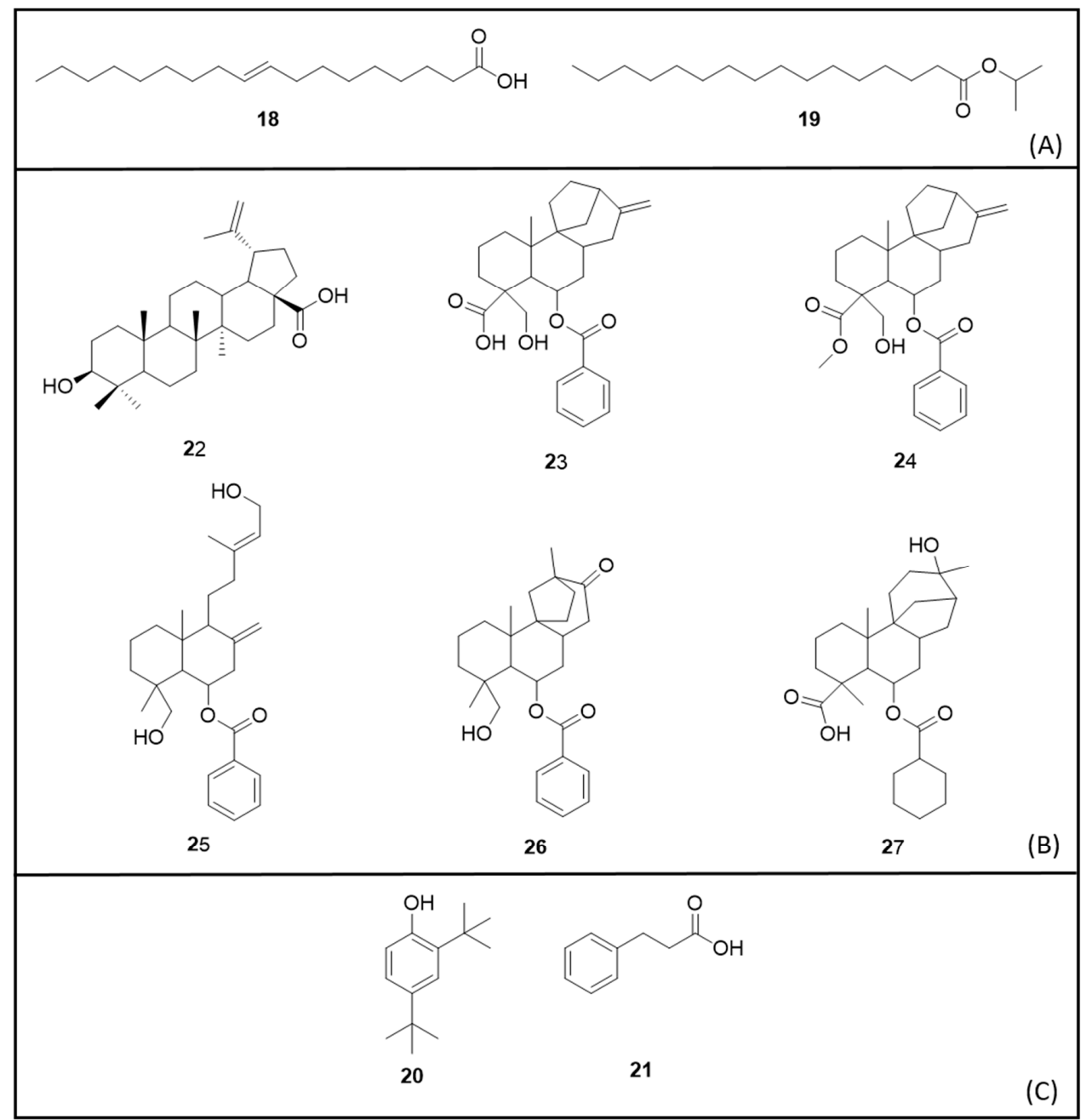

Figure 4. Secondary metabolites identified in organic extracts with insecticidal activity against Ae. aegypti. (A) Fatty acid and derivatives, (B) Diterpenes and triterpenes and (C) Others.

Tables 3 and 4 summarise the scientific literature selected for the discussion of insecticidal activities of organic/aqueous extracts against Ae. aeygpti mosquito. Table 3 describes larvicidal activities, while Table 4 describes adulticidal, pupicidal, ovicidal, repellent and oviposition activities. 
Table 3. Larvicidal activity of organic/aqueous extracts against the Ae. aegypti mosquito.

\begin{tabular}{|c|c|c|c|c|c|c|c|c|c|}
\hline \multirow{2}{*}{ Plant Species } & \multirow{2}{*}{ Family } & \multirow{2}{*}{ Country } & \multirow{2}{*}{ Part Used } & \multirow{2}{*}{ Extraction Solvent } & \multirow{2}{*}{ Larval Stage } & \multicolumn{2}{|c|}{ Mortality } & \multirow{2}{*}{$\begin{array}{c}\text { Time } \\
\text { (h) }\end{array}$} & \multirow{2}{*}{ Reference } \\
\hline & & & & & & $\mathrm{LC}_{50}(\mathrm{ppm})$ & $\mathrm{LC}_{90}(\mathrm{ppm})$ & & \\
\hline \multirow{7}{*}{ Acacia nilótica (L.) Delile } & \multirow{7}{*}{ Fabaceae } & \multirow{7}{*}{ India } & \multirow{6}{*}{ Seed pod } & $n$-Hexane & L4 & 169.25 & 201.62 & 24 & [58] \\
\hline & & & & Benzene & L4 & 45.32 & 99.32 & 24 & [58] \\
\hline & & & & Chloroform & L4 & 158.13 & 198.24 & 24 & [58] \\
\hline & & & & Ethyl acetate & L4 & 59.12 & 75.82 & 24 & [58] \\
\hline & & & & Acetone & L4 & 103.68 & 162.03 & 24 & [58] \\
\hline & & & & n-Hexane & L4 & 202.15 & 476.57 & 24 & [80] \\
\hline & & & \multirow{4}{*}{ Leaves } & Chloroform & L4 & 182.58 & 460.83 & 24 & {$[80]$} \\
\hline \multirow{3}{*}{ Acalypha alnifolia Klein ex Willd. } & \multirow{3}{*}{ Euphorbiaceae } & \multirow{3}{*}{ India } & & Ethyl acetate & L4 & 160.35 & 440.78 & 24 & [80] \\
\hline & & & & Acetone & L4 & 146.07 & 415.38 & 24 & [80] \\
\hline & & & & Methanol & L4 & 128.55 & 381.67 & 24 & [80] \\
\hline \multirow[t]{2}{*}{ Aristolochia bracteata Retz. } & \multirow[t]{2}{*}{ Aristolochiaceae } & \multirow[t]{2}{*}{ India } & \multirow[t]{2}{*}{ Leaves } & Methanol & L3 & 114.89 & 216.24 & 24 & [81] \\
\hline & & & & Water & L4-India & 117.18 & 227.63 & 24 & [82] \\
\hline \multirow[t]{3}{*}{ Artemisia herba-alba Asso } & \multirow[t]{3}{*}{ Asteraceae } & \multirow[t]{3}{*}{ Saudi Arabia } & \multirow[t]{3}{*}{ Leaves } & Water & $\begin{array}{l}\text { L4-Saudi } \\
\text { Arabia }\end{array}$ & 614.52 & 1273.33 & 24 & [82] \\
\hline & & & & Water + AgNP & L4-India & 10.70 & 21.24 & 24 & [82] \\
\hline & & & & Water + AgNP & $\begin{array}{l}\text { L4-Saudi } \\
\text { Arabia }\end{array}$ & 33.58 & 57.0 & 24 & [82] \\
\hline $\begin{array}{l}\text { Boenninghausenia albiflora (Hook.) Rchb. } \\
\text { ex Meisn. }\end{array}$ & Rutaceae & India & Leaves & Petroleum ether & L4 & 125.0 & 190.0 & ND & [83] \\
\hline Buddleja polystachya Fresen. & Buddlejaceae & Saudi Arabia & Flowers & n-Butanol & L1 & ND & ND & ND & [84] \\
\hline \multirow{3}{*}{ Caesalpinia pulcherrima (L.) Sw. } & & & & Ethyl acetate & L3 & 144.67 & 276.99 & 24 & [85] \\
\hline & Fabaceae & India & Leaves & Benzene & L3 & 136.36 & 272.15 & 24 & {$[85]$} \\
\hline & & & & Methanol & L3 & 10.69 & 20.47 & 24 & [86] \\
\hline Cassia fistula $\mathrm{L}$. & Fabaceae & India & Leaves & Benzene & L3 & 18.27 & 35.67 & 24 & [86] \\
\hline & & & & Acetone & L3 & 23.95 & 47.13 & 24 & [86] \\
\hline Catharanthus roseus (L.) G. Don & Apocynaceae & India & Leaves & Petroleum ether & L4 & 145.0 & 255.0 & 24 & [83] \\
\hline Cinnamosma fragrans Baill. & Canellaceae & Madagascar & Root Bark & Methanol & L1 & 52.5 & ND & 24 & [87] \\
\hline Citrullus colocynthis (L.) Schrad. & Cucurbitaceae & India & Leaves & Petroleum ether & L4 & 74.57 & 538.30 & 24 & [88] \\
\hline Cunninghamia konishii Hayata & & & Wood & Ethanol & $\mathrm{L} 4$ & 240.0 & $>400.0$ & 24 & [73] \\
\hline Cunninghamıa konishı Hayata & Taxodiaceae & Taiwan & Leaves & Ethanol & L4 & $>400.0$ & $>400.0$ & 24 & [73] \\
\hline & & & & Ethanol & L3 & 30.0 & 91.0 & 24 & [89] \\
\hline & & & & n-Hexane Fraction & L3 & 44.0 & 81.0 & 24 & [89] \\
\hline & & & Leaves & Chloroform Fraction & L3 & 33.0 & 75.0 & 24 & [89] \\
\hline Dalbergia brasiliensis Vogel & Fahaceae & & & Ethyl acetate Fraction & L3 & 24.0 & 66.0 & 24 & [89] \\
\hline 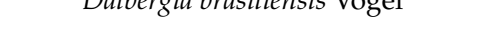 & Fabaceae & Brazil & & Ethanol & L3 & 32.0 & 71.0 & 24 & [89] \\
\hline & & & & n-Hexane Fraction & L3 & 31.0 & 72.0 & 24 & [89] \\
\hline & & & Bark & Chloroform Fraction & L3 & 25.0 & 50.0 & 24 & [89] \\
\hline
\end{tabular}


Table 3. Cont.

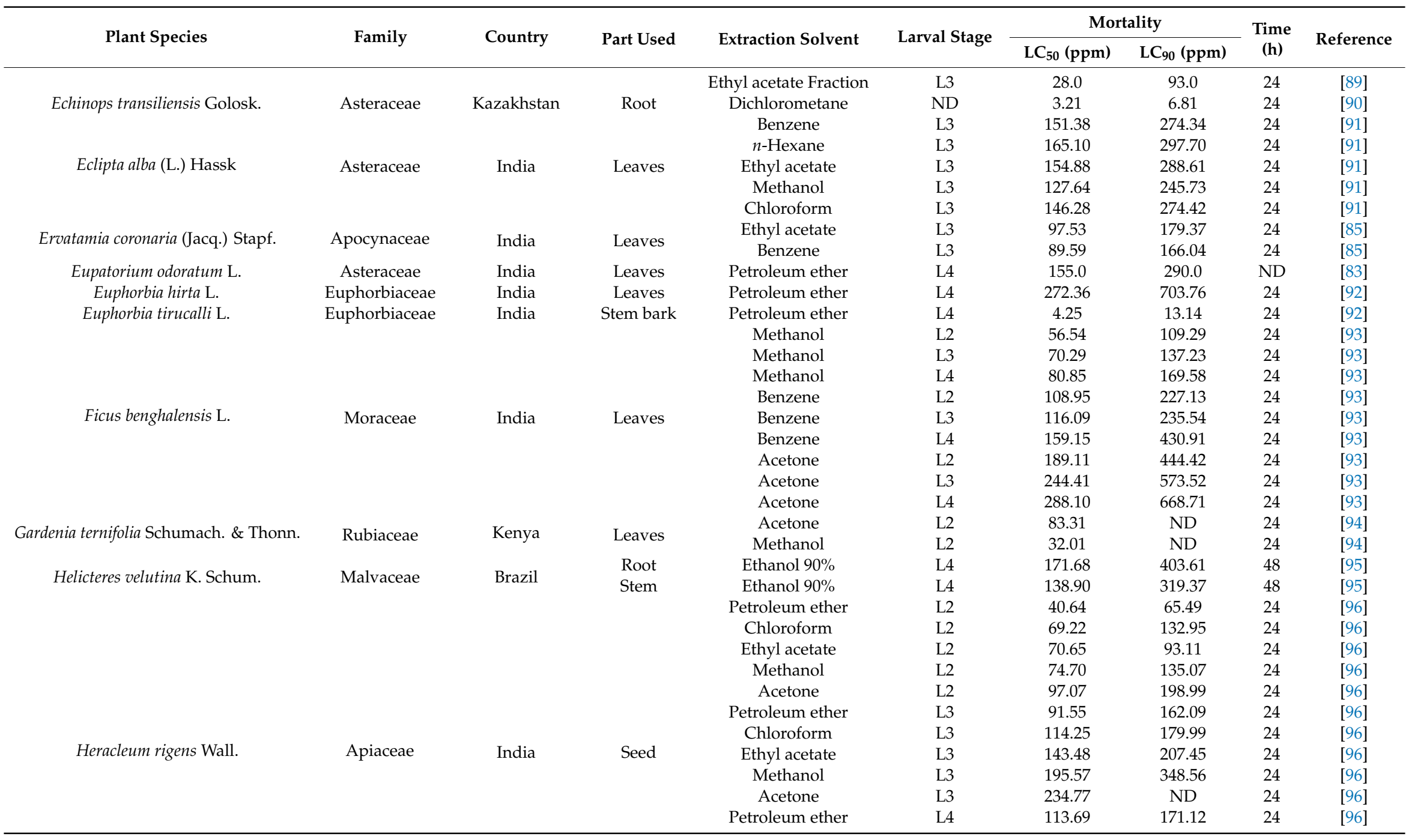


Table 3. Cont.

\begin{tabular}{|c|c|c|c|c|c|c|c|c|c|}
\hline \multirow{2}{*}{ Plant Species } & \multirow{2}{*}{ Family } & \multirow{2}{*}{ Country } & \multirow{2}{*}{ Part Used } & \multirow{2}{*}{ Extraction Solvent } & \multirow{2}{*}{ Larval Stage } & \multicolumn{2}{|c|}{ Mortality } & \multirow{2}{*}{$\begin{array}{c}\text { Time } \\
\text { (h) }\end{array}$} & \multirow{2}{*}{ Reference } \\
\hline & & & & & & $\mathrm{LC}_{50}(\mathrm{ppm})$ & $\mathrm{LC}_{90}(\mathrm{ppm})$ & & \\
\hline \multirow{8}{*}{ Hypericum japonicum Thunb. } & \multirow{8}{*}{ Hypericaceae } & \multirow{8}{*}{ India } & \multirow{8}{*}{ Whole plant } & Chloroform & L4 & 144.64 & 209.16 & 24 & [96] \\
\hline & & & & Ethyl acetate & L4 & 165.43 & 230.21 & 24 & [96] \\
\hline & & & & Methanol & L4 & 231.26 & 361.68 & 24 & [96] \\
\hline & & & & Acetone & L4 & 308.65 & 577.14 & 24 & [96] \\
\hline & & & & Acetone & L4 & 13.15 & 18.54 & 24 & [97] \\
\hline & & & & n-Hexane & L4 & 9.63 & 19.53 & 24 & [97] \\
\hline & & & & Petroleum ether & $\mathrm{L} 4$ & 8.27 & 15.2 & 24 & [97] \\
\hline & & & & Methanol & L4 & 7.37 & 11.59 & 24 & [97] \\
\hline \multirow[t]{2}{*}{ Jatropha curcas L. } & \multirow[t]{2}{*}{ Euphorbiaceae } & India & Leaves & Petroleum ether & $\mathrm{L} 4$ & 8.79 & 35.39 & 24 & [92] \\
\hline & & Indonesia & Root & Ethanol & ND & 44.75 & ND & 24 & {$[98]$} \\
\hline Limonia acidíssima $\mathrm{L}$. & Rutaceae & India & Leaves & $\begin{array}{l}n \text {-Hexane-Purified } \\
\text { fractions }\end{array}$ & L3 & 4.11 to 23.53 & ND & 24 & [99] \\
\hline \multirow[t]{2}{*}{ Lonchocarpus urucu Killip \& A.C. Sm. } & \multirow{2}{*}{ Fabaceae } & \multirow{2}{*}{ Brazil } & $\begin{array}{c}\text { Medulla } \\
\text { Root }\end{array}$ & Methanol & $\mathrm{L} 4$ & 33.32 & 83.69 & 24 & {$[100]$} \\
\hline & & & Bark Root & Methanol & L4 & 17.60 & 55.40 & 24 & {$[100]$} \\
\hline Maytenus oblongata Reissek & Celasteraceae & French Guiana & Bark & Ethyl acetate & L3/L4 & 74.40 & ND & 24 & [101] \\
\hline \multirow[t]{2}{*}{ Millettia pachycarpa Benth. } & Fabaceae & India & Root & Ethanol & L3 & 98.47 & ND & 24 & [102] \\
\hline & & & & Benzene & L3 & 97.03 & 172.15 & 24 & {$[103]$} \\
\hline \multirow{3}{*}{ Mirabilis jalapa L. } & \multirow{3}{*}{ Nyctaginaceae } & \multirow{3}{*}{ India } & \multirow{3}{*}{ Leaves } & Chloroform & L3 & 88.20 & 162.16 & 24 & [103] \\
\hline & & & & Ethyl acetate & L3 & 72.77 & 127.91 & 24 & [103] \\
\hline & & & & Methanol & L3 & 64.58 & 120.28 & 24 & [103] \\
\hline \multirow[t]{2}{*}{ Momordica charantia $\mathrm{L}$. } & \multirow[t]{2}{*}{ Cucurbitaceae } & \multirow[t]{2}{*}{ India } & \multirow[t]{2}{*}{ Leaves } & Methanol & $\mathrm{L} 4$ & 199.14 & 780.10 & 24 & {$[88]$} \\
\hline & & & & Methanol & L1 & 162.03 & 502.04 & 24 & {$[53]$} \\
\hline & & & & Methanol & L2 & 194.11 & 542.56 & 24 & {$[53]$} \\
\hline & & & & Methanol & L3 & 240.10 & 604.78 & 24 & [53] \\
\hline Myristica fragans Houtt & Myristicaceae & India ra & J & Methanol & $\mathrm{L} 4$ & 273.90 & 660.96 & 24 & [53] \\
\hline & & & & Methanol + ZnO NP & L1 & 3.44 & 18.35 & 24 & [53] \\
\hline & & & & Methanol + ZnO NP & L2 & 5.25 & 30.37 & 24 & [53] \\
\hline & & & & Methanol + ZnO NP & L3 & 8.02 & 39.14 & 24 & [53] \\
\hline & & & & Methanol + ZnO NP & L4 & 10.28 & 44.07 & 24 & [53] \\
\hline Myristica fragrans Houtt. & Myristicaceae & Thailand & Flowers & Ethanol & L4 (p-s) & 75.45 & 123.60 & 24 & [60] \\
\hline Nerine sarniensis (L.) Herb. & Amaryllidaceae & South Africa & Bulb & Ethyl acetate & L1 & 8.0 & ND & 24 & [104] \\
\hline Nyctanthes arbor-tristis L. & Oleaceae & India & Leaves & Petroleum ether & L4 & 180.0 & 340.0 & & [83] \\
\hline Ocimum sanctum $\mathrm{L}$. & Labiatae & India & Leaves and & Acetone & L4 & 425.94 & ND & 24 & [105] \\
\hline & & & & Chloroform & L4 & 150.40 & ND & 24 & [105] \\
\hline
\end{tabular}


Table 3. Cont.

\begin{tabular}{|c|c|c|c|c|c|c|c|c|c|}
\hline \multirow{2}{*}{ Plant Species } & \multirow{2}{*}{ Family } & \multirow{2}{*}{ Country } & \multirow{2}{*}{ Part Used } & \multirow{2}{*}{ Extraction Solvent } & \multirow{2}{*}{ Larval Stage } & \multicolumn{2}{|c|}{ Mortality } & \multirow{2}{*}{$\begin{array}{c}\text { Time } \\
\text { (h) }\end{array}$} & \multirow{2}{*}{ Reference } \\
\hline & & & & & & $\mathrm{LC}_{50}$ (ppm) & $\mathrm{LC}_{90}(\mathrm{ppm})$ & & \\
\hline \multirow{7}{*}{ Ormosia arborea Vell } & \multirow{7}{*}{ Fabaceae } & \multirow{7}{*}{ Brazil } & \multirow{7}{*}{$\begin{array}{l}\text { Leaves } \\
\text { Seeds }\end{array}$} & Ethyl acetate & L4 & 350.78 & ND & 24 & [105] \\
\hline & & & & $n$-Hexane & L4 & 575.26 & ND & 24 & [105] \\
\hline & & & & Methanol & L4 & 175.67 & ND & 24 & [105] \\
\hline & & & & Ethanol & L3 & 238.0 & 347.0 & 24 & [106] \\
\hline & & & & Ethanol & L3 & 111.0 & 194.0 & 24 & [106] \\
\hline & & & & n-Hexane & L3 & 228.13 & 526.12 & 24 & [107] \\
\hline & & & & Chloroform & L3 & 209.72 & 502.84 & 24 & [107] \\
\hline \multirow[t]{3}{*}{ Orthosiphon thymiflorus (Roth) Sleesen } & \multirow[t]{3}{*}{ Labiatae } & \multirow[t]{3}{*}{ India } & \multirow[t]{3}{*}{ Leaves } & Ethyl acetate & L3 & 183.35 & 463.35 & 24 & [107] \\
\hline & & & & Acetone & L3 & 163.55 & 442.32 & 24 & [107] \\
\hline & & & & Methanol & L3 & 149.96 & 426.16 & 24 & [107] \\
\hline \multirow[t]{2}{*}{ Pedilanthus tithymaloides (L.) Poit. } & \multirow[t]{2}{*}{ Euphorbiaceae } & \multirow[t]{2}{*}{ India } & \multirow[t]{2}{*}{ Leaves } & Petroleum ether & L4 & 55.26 & 256.77 & 24 & {$[92]$} \\
\hline & & & & Methanol & L3 & 22.10 & 43.71 & 24 & [108] \\
\hline \multirow[t]{2}{*}{ Pemphis acidula J.R. Forst. \& G. Forst. } & \multirow[t]{2}{*}{ Lythraceae } & \multirow[t]{2}{*}{ India } & \multirow[t]{2}{*}{ Leaves } & Benzene & L3 & 43.99 & 84.87 & 24 & [108] \\
\hline & & & & Acetone & L3 & 57.66 & 106.51 & 24 & [108] \\
\hline $\begin{array}{c}\text { Phyllanthus amarus Schumach. \& } \\
\text { Thonn. }\end{array}$ & Euphorbiaceae & India & Leaves & Petroleum ether & $\mathrm{L} 4$ & 90.92 & 384.19 & 24 & [92] \\
\hline \multirow{2}{*}{ Piper aduncum $\mathrm{L}$. } & \multirow{2}{*}{ Piperaceae } & Brazil & Jeaves & n-Hexane & L3 & 342.0 & 473.0 & 24 & [106] \\
\hline & & Brazil & Leaves & Chloroform & L3 & 192.0 & 346.0 & 24 & [106] \\
\hline Piper hispidum Sw. & Piperaceae & & & Ethanol & L3 & 169.0 & 474.0 & 24 & [106] \\
\hline Ptper nispiaum sw. & Piperaceae & Brazil & Leaves & Chloroform & L3 & 567.0 & 1003.0 & 24 & [106] \\
\hline Piper longum L. & Piperaceae & Thailand & Fruits & Ethanol & L4 & 2.23 & ND & 24 & [109] \\
\hline & & & & Ethanol & L3/L4 & 71.25 & 9.37 & 24 & [110] \\
\hline Piper nigrum L. & Piperaceae & Philippines & Peppercorns & Ethanol-Fraction 1A & $\mathrm{L} 3 / \mathrm{L} 4$ & 17.10 & 3.84 & 24 & [110] \\
\hline & & & & Ethanol一Fraction 1B & $\mathrm{L} 3 / \mathrm{L} 4$ & 18.10 & 3.84 & 24 & [110] \\
\hline Piper ribesoides Wall. & Piperaceae & Thailand & Wood & Ethanol & L4 & 8.13 & ND & 24 & [109] \\
\hline Piper sarmentosum Roxb. ex Hunt. & Piperaceae & Thailand & Whole plant & Ethanol & L4 & 4.06 & ND & 24 & [109] \\
\hline & & & & Methanol & L3 & 155.78 & 279.73 & 24 & [111] \\
\hline & & & & Ethyl acetate & L3 & 162.36 & 283.43 & 24 & [111] \\
\hline & & & Leaves & Chloroform & L3 & 169.08 & 293.17 & 24 & [111] \\
\hline & & & & Benzene & L3 & 176.02 & 308.88 & 24 & [111] \\
\hline Pithecellobium Dulce (Roxb.) Benth. & Fabaceae & India & & n-Hexane & L3 & 185.14 & 316.46 & 24 & [111] \\
\hline & randcede & Hinta & & Methanol & L3 & 193.66 & 377.39 & 24 & [111] \\
\hline & & & & Ethyl acetate & L3 & 215.63 & 416.51 & 24 & [111] \\
\hline & & & Seeds & Chloroform & L3 & 240.39 & 461.28 & 24 & [111] \\
\hline
\end{tabular}


Table 3. Cont.

\begin{tabular}{|c|c|c|c|c|c|c|c|c|c|}
\hline \multirow{2}{*}{ Plant Species } & \multirow{2}{*}{ Family } & \multirow{2}{*}{ Country } & \multirow{2}{*}{ Part Used } & \multirow{2}{*}{ Extraction Solvent } & \multirow{2}{*}{ Larval Stage } & \multicolumn{2}{|c|}{ Mortality } & \multirow{2}{*}{$\begin{array}{l}\text { Time } \\
\text { (h) }\end{array}$} & \multirow{2}{*}{ Reference } \\
\hline & & & & & & $\mathrm{LC}_{50}$ (ppm) & $\mathrm{LC}_{90}$ (ppm) & & \\
\hline \multirow{5}{*}{$\begin{array}{l}\text { Scoparia dulcis L. } \\
\text { Solanum nigrum L. }\end{array}$} & \multirow{4}{*}{ Plantaginaceae } & \multirow[b]{3}{*}{ Brazil } & \multirow[b]{3}{*}{ Leaves } & Benzene & L3 & 259.42 & 489.41 & 24 & [111] \\
\hline & & & & n-Hexane & L3 & 281.18 & 516.33 & 24 & [111] \\
\hline & & & & Ethanol $90 \%$ & L4 & 83.43 & 158.83 & 48 & [112] \\
\hline & & \multirow{4}{*}{$\begin{array}{l}\text { India } \\
\text { Brazil }\end{array}$} & \multirow{2}{*}{ Fruit } & Water & L3/L4 & 359.0 & 931.0 & 24 & [113] \\
\hline & \multirow{3}{*}{ Solanaceae } & & & n-Hexane & $\mathrm{L} 3 / \mathrm{L} 4$ & 17.63 & 65.22 & 24 & [113] \\
\hline \multirow[t]{2}{*}{ Solanum variabile Mart. } & & & Leaves & Ethanol & L3 & 188.0 & 284.0 & 24 & [106] \\
\hline & & & \multirow{4}{*}{ Fruit } & Methanol & L1 & 170.91 & 320.62 & 24 & [114] \\
\hline \multirow{3}{*}{$\begin{array}{c}\text { Solanum xanthocarpum Schrad. \& J.C. } \\
\text { Wendl. }\end{array}$} & \multirow{3}{*}{ Solanaceae } & \multirow{3}{*}{ India } & & Methanol & L2 & 195.07 & 366.48 & 24 & [114] \\
\hline & & & & Methanol & L3 & 221.45 & 410.20 & 24 & [114] \\
\hline & & & & Methanol & L4 & 253.18 & 435.16 & 24 & [114] \\
\hline \multirow{2}{*}{ Spermacoce latifólia Aubl. } & \multirow{2}{*}{ Rubiaceae } & \multirow{2}{*}{ Brazil } & \multirow{2}{*}{ Leaves } & n-Hexane & L3 & 415.0 & 901.0 & 24 & [106] \\
\hline & & & & Methanol & L3 & 625.0 & 1122.0 & 24 & [106] \\
\hline \multirow{2}{*}{ Tagetes patula $\mathrm{L}$. } & \multirow{2}{*}{ Asteraceae } & \multirow{2}{*}{ Brazil } & \multirow{2}{*}{ Seeds } & Acetone & L4 & 15.74 & ND & 48 & [115] \\
\hline & & & & Ethanol $50 \%$ & L4 & 25.46 & ND & 48 & [115] \\
\hline Turnera ulmifolia L. & Turneracea & Brazil & Leaves & Ethanol & L3 & 242.0 & 899.0 & 24 & [106] \\
\hline Valeriana hardwickii Wall. & Valerianaceae & India & Leaves & Petroleum ether & L4 & 235.0 & 415.0 & ND & [83] \\
\hline \multirow{3}{*}{ Ventilago madraspatana Gaertn. } & \multirow{2}{*}{ Rhammnaceae } & \multirow{2}{*}{ India } & \multirow{2}{*}{ Leaves } & Water + AgNP & L3 & 26.92 & ND & 24 & [112] \\
\hline & & & & Water & L3 & 267.27 & ND & 24 & [112] \\
\hline & Orchidaceae & India & Leaves & Water + AgNP & L3 & 10.39 & 23.58 & 24 & [116] \\
\hline
\end{tabular}

$\mathrm{LC}_{50}$ lethal concentration required to kill $50 \%$ of the larval population, $\mathrm{LC}_{90}$ lethal concentration required to kill $90 \%$ of the larval population, ND not described, $\mathrm{p}$-s pyrethroid-susceptible,

AgNP silver nanoparticle, ZnONP zinc oxide nanoparticle. 
Table 4. Adulticidal, pupicidal, ovicidal, repellent and oviposition activities of organic/aqueous extracts against the Ae. aegypti mosquito.

\begin{tabular}{|c|c|c|c|c|c|c|c|c|}
\hline Plant Species & Family & Country & Part Used & $\begin{array}{l}\text { Extraction } \\
\text { Solvent }\end{array}$ & Activity & Results & $\begin{array}{c}\text { Time } \\
\text { (h) }\end{array}$ & Reference \\
\hline \multirow{2}{*}{$\begin{array}{l}\text { Alpinia purpurata (Viell.) K. } \\
\text { Schum. }\end{array}$} & \multirow{2}{*}{ Zingiberaceae } & \multirow{2}{*}{ Brazil } & Red Flowers & Water & Oviposition & Oviposition disruptive effect & 24 & [67] \\
\hline & & & Pink Flowers & Water & Oviposition & Oviposition disruptive effect & 24 & [67] \\
\hline \multirow{3}{*}{ Aristolochia bracteata Retz. } & \multirow{2}{*}{ Aristolochiaceae } & \multirow{2}{*}{ India } & \multirow{2}{*}{ Leaves } & Methanol & Ovicide & Zero hatchability at $240 \mathrm{ppm}$ & 48 & [81] \\
\hline & & & & Methanol & Repellent & $100 \%$ of repellency at $6 \mathrm{mg} / \mathrm{cm}^{2}$ & 3 & [81] \\
\hline & \multirow{4}{*}{ Asteraceae } & \multirow{5}{*}{ Saudi Arabia } & \multirow{4}{*}{ Leaves } & Water & $\begin{array}{l}\text { Adulticide—strain } \\
\text { from India }\end{array}$ & $\begin{array}{c}\mathrm{LC}_{50} 327.15 \mu \mathrm{g} / \mathrm{mL} \\
\mathrm{LC}_{90}=779.98 \mu \mathrm{g} / \mathrm{mL}\end{array}$ & 24 & {$[82]$} \\
\hline \multirow[t]{4}{*}{ Artemisia herba-alba Asso } & & & & Water & $\begin{array}{l}\text { Adulticide-strain } \\
\text { from Saudi Arabia }\end{array}$ & $\begin{array}{l}\mathrm{LC}_{50} 450.21 \mu \mathrm{g} / \mathrm{mL} \\
\mathrm{LC}_{90} 1153.18 \mu \mathrm{g} / \mathrm{mL}\end{array}$ & 24 & {$[82]$} \\
\hline & & & & Water + AgNP & $\begin{array}{l}\text { Adulticide—strain } \\
\text { from India }\end{array}$ & $\begin{array}{l}\mathrm{LC}_{50} 8.71 \mu \mathrm{g} / \mathrm{mL} \\
\mathrm{LC}_{90} 39.88 \mu \mathrm{g} / \mathrm{mL}\end{array}$ & 24 & {$[82]$} \\
\hline & & & & Water + AgNP & $\begin{array}{l}\text { Adulticide-strain } \\
\text { from Saudi Arabia }\end{array}$ & $\begin{array}{l}\mathrm{LC}_{50} 25.62 \mu \mathrm{g} / \mathrm{mL} \\
\mathrm{LC}_{90} 48.88 \mu \mathrm{g} / \mathrm{mL}\end{array}$ & 24 & [82] \\
\hline & \multirow{6}{*}{ Buddlejaceae } & & \multirow{2}{*}{ Flowers } & n-Hexane & Adulticide & $96.7 \%$ mortality at $5 \mu \mathrm{g} / \mathrm{mg}$ female & ND & [84] \\
\hline \multirow{5}{*}{ Buddleja polystachya Fresen. } & & \multirow{5}{*}{ Saudi Arabia } & & Ethanol & Adulticide & $83.3 \%$ mortality at $5 \mu \mathrm{g} / \mathrm{mg}$ female & ND & [84] \\
\hline & & & \multirow{4}{*}{ Aerial parts } & $n$-Hexane & Adulticide & $100 \%$ mortality at $5 \mu \mathrm{g} / \mathrm{mg}$ female & ND & [84] \\
\hline & & & & Ethanol & Adulticide & $90 \%$ mortality at $5 \mu \mathrm{g} / \mathrm{mg}$ female & ND & [84] \\
\hline & & & & Methanol & Ovicide & Zero hatchability at 300 ppm & 48 & {$[85,117]$} \\
\hline & & & & Methanol & Repellent & $100 \%$ of repellency at $5 \mathrm{mg} / \mathrm{cm}^{2}$ & 3 & {$[85,114]$} \\
\hline \multirow[t]{6}{*}{ Caesalpinia pulcherrima (L.) Sw. } & \multirow[t]{6}{*}{ Fabaceae } & \multirow[t]{6}{*}{ India } & \multirow[t]{6}{*}{ Leaves } & Ethyl acetate & Ovicide & Zero hatchability at $450 \mathrm{ppm}$ & 48 & {$[85,117]$} \\
\hline & & & & Ethyl acetate & Repellent & $100 \%$ of repellency at $5 \mathrm{mg} / \mathrm{cm}^{2}$ & 1.5 & {$[85,117]$} \\
\hline & & & & Benzene & Ovicide & Zero hatchability at 375 ppm & 48 & {$[85,117]$} \\
\hline & & & & Benzene & Repellent & $100 \%$ of repellency at $5 \mathrm{mg} / \mathrm{cm}^{2}$ & 2 & {$[85,117]$} \\
\hline & & & & Methanol & Repellent & $100 \%$ of repellency at $5 \mathrm{mg} / \mathrm{cm}^{2}$ & 3 & [118] \\
\hline & & & & n-Hexane & Repellent & $100 \%$ of repellency at $5 \mathrm{mg} / \mathrm{cm}^{2}$ & 3 & [118] \\
\hline \multirow[t]{5}{*}{ Cardiospermum halicacabum $L$. } & \multirow[t]{5}{*}{ Sapindaceae } & \multirow[t]{5}{*}{ India } & \multirow[t]{5}{*}{ Leaves } & Ethyl acetate & Repellent & $100 \%$ of repellency at $5 \mathrm{mg} / \mathrm{cm}^{2}$ & 3 & [118] \\
\hline & & & & Chloroform & Repellent & $100 \%$ of repellency at $5 \mathrm{mg} / \mathrm{cm}^{2}$ & 3 & [118] \\
\hline & & & & Benzene & Repellent & $100 \%$ of repellency at $5 \mathrm{mg} / \mathrm{cm}^{2}$ & 3 & [118] \\
\hline & & & & Methanol & Ovicide & Zero hatchability at $120 \mathrm{ppm}$ & 48 & [86] \\
\hline & & & & Methanol & Repellent & $100 \%$ of repellency at $5 \mathrm{mg} / \mathrm{cm}^{2}$ & 6 & [86] \\
\hline \multirow{4}{*}{ Cassia fistula L. } & \multirow{4}{*}{ Fabaceae } & \multirow{4}{*}{ India } & \multirow{4}{*}{ Leaves } & Benzene & Ovicide & Zero hatchability at $140 \mathrm{ppm}$ & 48 & [86] \\
\hline & & & & Benzene & Repellent & $100 \%$ of repellency at $5 \mathrm{mg} / \mathrm{cm}^{2}$ & 5 & [86] \\
\hline & & & & Acetone & Ovicide & Zero hatchability at $160 \mathrm{ppm}$ & 48 & [86] \\
\hline & & & & Acetone & Repellent & $100 \%$ of repellency at $5 \mathrm{mg} / \mathrm{cm}^{2}$ & 4.3 & [86] \\
\hline \multirow{2}{*}{ Cinnamosma fragrans Baill. } & Canollaceа & Madagascar & Root harks & Methanol & Adulticide & $\mathrm{LC}_{50} 0.17 \mu \mathrm{g} / \mathrm{mg}$ female & 24 & [87] \\
\hline & Canellaceae & & Koot barks & Methanol & Repellent & $80 \%$ of repellency at $20.8 \mu \mathrm{g} / \mathrm{cm}^{2}$ & 3 & [87] \\
\hline
\end{tabular}


Table 4. Cont.

\begin{tabular}{|c|c|c|c|c|c|c|c|c|}
\hline Plant Species & Family & Country & Part Used & $\begin{array}{l}\text { Extraction } \\
\text { Solvent }\end{array}$ & Activity & Results & $\begin{array}{l}\text { Time } \\
\text { (h) }\end{array}$ & Reference \\
\hline \multirow{12}{*}{ Coccinia indica Wight \& Arn. } & \multirow{12}{*}{ Cucurbitaceae } & \multirow{12}{*}{ India } & \multirow{12}{*}{ Leaves } & Benzene & Ovicide & Zero hatchability at $250 \mathrm{ppm}$ & 48 & [119] \\
\hline & & & & Benzene & Repellent & $100 \%$ of repellency at $5 \mathrm{mg} / \mathrm{cm}^{2}$ & 3 & [119] \\
\hline & & & & $n$-Hexane & Ovicide & Zero hatchability at $300 \mathrm{ppm}$ & 48 & [119] \\
\hline & & & & n-Hexane & Repellent & $100 \%$ of repellency at $1 \mathrm{mg} / \mathrm{cm}^{2}$ & 2.5 & [119] \\
\hline & & & & Ethyl acetate & Ovicide & Zero hatchability at $250 \mathrm{ppm}$ & 48 & [119] \\
\hline & & & & Ethyl acetate & Repellent & $100 \%$ of repellency at $2.5 \mathrm{mg} / \mathrm{cm}^{2}$ & 2.5 & [119] \\
\hline & & & & Methanol & Ovicide & Zero hatchability at $200 \mathrm{ppm}$ & 48 & [119] \\
\hline & & & & Methanol & Repellent & $100 \%$ of repellency at $5 \mathrm{mg} / \mathrm{cm}^{2}$ & 3.5 & [119] \\
\hline & & & & Chloroform & Ovicide & Zero hatchability at $250 \mathrm{ppm}$ & 48 & [119] \\
\hline & & & & Chloroform & Repellent & $100 \%$ of repellency at $2.5 \mathrm{mg} / \mathrm{cm}^{2}$ & 2.5 & [119] \\
\hline & & & & Benzene & Ovicide & Zero hatchability at $350 \mathrm{ppm}$ & 48 & [119] \\
\hline & & & & n-Hexane & Ovicide & $21 \%$ hatchability at $350 \mathrm{ppm}$ & 48 & [119] \\
\hline \multirow[t]{5}{*}{ Eclipta alba (L.) Hassk } & \multirow[t]{5}{*}{ Asteraceae } & \multirow[t]{5}{*}{ India } & \multirow[t]{5}{*}{ Leaves } & Ethyl acetate & Ovicide & Zero hatchability at $350 \mathrm{ppm}$ & 48 & [119] \\
\hline & & & & Methanol & Ovicide & Zero hatchability at $300 \mathrm{ppm}$ & 48 & [91] \\
\hline & & & & Chloroform & Ovicide & Zero hatchability at $350 \mathrm{ppm}$ & 48 & [119] \\
\hline & & & & Methanol & Ovicide & Zero hatchability at $200 \mathrm{ppm}$ & 48 & [117] \\
\hline & & & & Methanol & Repellent & $100 \%$ of repellency at $5 \mathrm{mg} / \mathrm{cm}^{2}$ & 3 & [117] \\
\hline \multirow{4}{*}{ Ervatamia coronaria (Jacq.) Stapf. } & \multirow{4}{*}{ Apocynaceae } & \multirow{4}{*}{ India } & \multirow{4}{*}{ Leaves } & Ethyl acetate & Ovicide & Zero hatchability at $300 \mathrm{ppm}$ & 48 & [117] \\
\hline & & & & Ethyl acetate & Repellent & $100 \%$ of repellency at $5 \mathrm{mg} / \mathrm{cm}^{2}$ & 2 & [117] \\
\hline & & & & Benzene & Ovicide & Zero hatchability at $250 \mathrm{ppm}$ & 48 & [117] \\
\hline & & & & Benzene & Repellent & $100 \%$ of repellency at $5 \mathrm{mg} / \mathrm{cm}^{2}$ & 2.5 & [117] \\
\hline \multirow[t]{2}{*}{ Limonia acidíssima L. } & \multirow[t]{2}{*}{ Rutaceae } & \multirow[t]{2}{*}{ India } & \multirow[t]{2}{*}{ Leaves } & $\begin{array}{l}n \text {-Hexane-Purified } \\
\text { fractions }\end{array}$ & Ovicide & 78.4 hatchability at $10 \mathrm{ppm}$ & 120 & [99] \\
\hline & & & & $\begin{array}{l}\text { n-Hexane-Purified } \\
\text { fractions }\end{array}$ & Pupicide & $\mathrm{LC}_{50} 4.19-39.48 \mu \mathrm{g} / \mathrm{mL}$ & 24 & [99] \\
\hline Mentha piperita L. & Lamiaceae & India & Whole plant & Methanol & Repellent & Repellency & ND & [120] \\
\hline Millettia pachycarpa Benth. & Fabaceae & India & Root & Ethanol & Ovicide & Zero hatchability at $200 \mathrm{ppm}$ & 24 & [102] \\
\hline \multirow{4}{*}{ Myristica fragans Houtt. } & \multirow{4}{*}{ Myristicaceae } & \multirow{4}{*}{ India } & \multirow{4}{*}{ Leaves } & Methanol & Pupicide & $\begin{array}{l}\mathrm{LC}_{50} 359.08 \mu \mathrm{g} / \mathrm{mL} \\
\mathrm{LC}_{90} 803.52 \mu \mathrm{g} / \mathrm{mL}\end{array}$ & 24 & {$[53]$} \\
\hline & & & & $\begin{array}{l}\text { Methanol + } \\
\text { ZnONP }\end{array}$ & Pupicide & $\begin{array}{l}\mathrm{LC}_{50} 14.63 \mu \mathrm{g} / \mathrm{mL} \\
\mathrm{LC}_{90} 51.22 \mu \mathrm{g} / \mathrm{mL}\end{array}$ & 24 & [53] \\
\hline & & & & Methanol & Adulticide & $\begin{array}{l}\mathrm{LC}_{50} 180.26 \mu \mathrm{g} / \mathrm{mL} \\
\mathrm{LC}_{90} 368.93 \mu \mathrm{g} / \mathrm{mL}\end{array}$ & 24 & [53] \\
\hline & & & & $\begin{array}{l}\text { Methanol + } \\
\text { ZnONP }\end{array}$ & Adulticide & $\begin{array}{l}\mathrm{LC}_{50} 15.0 \mu \mathrm{g} / \mathrm{mL} \\
\mathrm{LC}_{90} 34.2 \mu \mathrm{g} / \mathrm{mL}\end{array}$ & 24 & [53] \\
\hline Nerine sarniensis (L.) Herb. & Amaryllidaceae & South Africa & Bulbs & Ehtyl acetate & Adulticide & $\mathrm{LC}_{50} 4.6 \mu \mathrm{g} / \mathrm{mg}$ female & 24 & [104] \\
\hline
\end{tabular}


Table 4. Cont.

\begin{tabular}{|c|c|c|c|c|c|c|c|c|}
\hline Plant Species & Family & Country & Part Used & $\begin{array}{l}\text { Extraction } \\
\text { Solvent }\end{array}$ & Activity & Results & $\begin{array}{l}\text { Time } \\
\text { (h) }\end{array}$ & Reference \\
\hline \multirow{4}{*}{ Parthenium hysterophorus } & \multirow{4}{*}{ Asteraceae } & \multirow{4}{*}{ India } & \multirow{4}{*}{ Leaves } & Ether & Repellent & $99.6 \%$ of repellency at $1000 \mathrm{ppm}$ & 48 & [121] \\
\hline & & & & Ether & Ovicide & Zero hatchability at $1000 \mathrm{ppm}$ & 48 & [121] \\
\hline & & & & Benzene & Repellent & $93.8 \%$ of repellency at $1000 \mathrm{ppm}$ & 48 & [121] \\
\hline & & & & Benzene & Ovicide & Zero hatchability at $1000 \mathrm{ppm}$ & 48 & [121] \\
\hline \multirow{3}{*}{$\begin{array}{l}\text { Pemphis acidula J.R. Forst. \& G. } \\
\text { Forst. }\end{array}$} & \multirow{3}{*}{ Lythraceae } & \multirow{3}{*}{ India } & \multirow{3}{*}{ Leaves } & Methanol & Ovicide & Zero hatchability at $450 \mathrm{ppm}$ & 48 & [108] \\
\hline & & & & Acetone & Ovicide & Zero hatchability at $500 \mathrm{ppm}$ & 48 & [108] \\
\hline & & & & Methanol & Ovicide & Zero hatchability at $400 \mathrm{ppm}$ & 48 & [111] \\
\hline \multirow{8}{*}{$\begin{array}{l}\text { Pithecellobium Dulce (Roxb.) } \\
\text { Benth. }\end{array}$} & \multirow{8}{*}{ Fabaceae } & \multirow{8}{*}{ India } & \multirow{3}{*}{ Leaves } & Ehtyl acetate & Ovicide & Zero hatchability at $500 \mathrm{ppm}$ & 48 & [111] \\
\hline & & & & Chloroform & Ovicide & Zero hatchability at $500 \mathrm{ppm}$ & 48 & [111] \\
\hline & & & & Benzene & Ovicide & Zero hatchability at $600 \mathrm{ppm}$ & 48 & [111] \\
\hline & & & & $n$-Hexane & Ovicide & Zero hatchability at $600 \mathrm{ppm}$ & 48 & [111] \\
\hline & & & & Methanol & Ovicide & Zero hatchability at $625 \mathrm{ppm}$ & 48 & [111] \\
\hline & & & & Ethyl acetate & Ovicide & Zero hatchability at $750 \mathrm{ppm}$ & 48 & [111] \\
\hline & & & Seeds & Chloroform & Ovicide & Zero hatchability at $750 \mathrm{ppm}$ & 48 & [111] \\
\hline & & & & Benzene & Ovicide & Zero hatchability at $750 \mathrm{ppm}$ & 48 & [111] \\
\hline \multirow[t]{2}{*}{$\begin{array}{c}\text { Solanum xanthocarpum Schrad. } \\
\text { \& J.C. Wendl. }\end{array}$} & \multirow[t]{2}{*}{ Solanaceae } & \multirow[t]{2}{*}{ India } & \multirow[t]{2}{*}{ Fruit } & Methanol & Pupicide & $\begin{array}{l}\mathrm{LC}_{50} 279.52 \mu \mathrm{g} / \mathrm{mL} \\
\mathrm{LC}_{90} 462.10 \mu \mathrm{g} / \mathrm{mL}\end{array}$ & 24 & [114] \\
\hline & & & & Water + AgNP & Ovicide & Zero hatchability at $120 \mathrm{ppm}$ & 48 & [112] \\
\hline \multirow{3}{*}{ Ventilago madraspatana Gaertn. } & \multirow{3}{*}{ Rhammnaceae } & \multirow{3}{*}{ India } & \multirow{3}{*}{ Leaves } & Water & Ovicide & Zero hatchability at $400 \mathrm{ppm}$ & 48 & [112] \\
\hline & & & & Water + AgNP & Adulticide & $\mathrm{LC}_{50} 44.85 \mu \mathrm{g} / \mathrm{mL}$ & 24 & [112] \\
\hline & & & & Water & Adulticide & $\mathrm{LC}_{50} 334.46 \mu \mathrm{g} / \mathrm{mL}$ & 24 & [112] \\
\hline \multirow{2}{*}{ Zeuxine gracilis (Berda) Bl. } & \multirow{2}{*}{ Orchidaceae } & \multirow{2}{*}{ India } & \multirow{2}{*}{ Leaves } & Water + AgNP & Ovicide & Zero hatchability at $12 \mathrm{ppm}$ & 48 & [116] \\
\hline & & & & 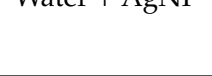 & Adulticide & $\begin{array}{c}\mathrm{LC}_{50} 27.90 \mu \mathrm{g} / \mathrm{mL} \\
\mathrm{LC}_{90}=59.20 \mu \mathrm{g} / \mathrm{mL}\end{array}$ & 24 & [116] \\
\hline
\end{tabular}

$\mathrm{LC}_{50}$ lethal concentration required to to kill $50 \%$ of the mosquito population, $\mathrm{LC}_{90}$ lethal concentration required to kill $90 \%$ of the mosquito population, ND not described, AgNP silver nanoparticle, ZnONP zinc oxide nanoparticle. 
Piper species (Piperaceae) demonstrated $\mathrm{LC}_{50}$ ranging from 2.23 to $567 \mu \mathrm{g} / \mathrm{mL}$ for $\mathrm{L} 3$ and $\mathrm{L} 4$ larval stages $[106,109,110]$. The most active species extracts were Piper longum L. (fruit ethanolic), followed by P. sarmentosum (entire plant ethanolic $\mathrm{LC}_{50} 4.06 \mu \mathrm{g} / \mathrm{mL}$ ) and Piper ribesoides Wall. (wood $\mathrm{LC}_{50} 8.13 \mu \mathrm{g} / \mathrm{mL}$ ) [109]. Piper nigrum L. peppercorn ethanolic extract was active and purified fractions were highly active, with possible toxicity due to oleic acid (18) [110]. P. aduncum and Piper hispidum Sw. displayed weak activity against $\mathrm{L} 3$ larvae $\left(\mathrm{LC}_{50}>150 \mu \mathrm{g} / \mathrm{mL}\right)$ [106].

An Echinops transiliensis Golosk. (Asteraceae) root dichloromethane extract showed strong toxicity against larvae $\left(\mathrm{LC}_{50} 3.21 \mu \mathrm{g} / \mathrm{mL}\right)$. The nine thiophene derivatives isolated showed strong activity $\left(\mathrm{LC}_{50}\right.$ 0.16-19.97 $\mu \mathrm{g} / \mathrm{mL}$ ) [90]. Similarly, a Euphorbia tirucalli L. (Euphorbiaceae) stem bark petroleum ether extract presented $\mathrm{LC}_{50} 4.25 \mu \mathrm{g} / \mathrm{mL}$ against L4 larvae, while a Euphorbia hirta L. leaves extract exhibited weak activity $\left(\mathrm{LC}_{50} 272.36 \mu \mathrm{g} / \mathrm{mL}\right)$ [92].

The Hypericum japonicum Thunb. (Hypericaceae) species was also very toxic to mosquito larvae. The organic extracts of the whole plant presented $\mathrm{LC}_{50}$ values between 7.37 and $13.15 \mu \mathrm{g} / \mathrm{mL}$. The methanolic extract proved the most active, its purified fraction displaying $\mathrm{LC}_{50} 0.95 \mu \mathrm{g} / \mathrm{mL}$. The major constituents of this active fraction were isopropyl palmitate (19), 2,4-Di-tert-butylphenol (20) and hydrocinnamic acid (21) [97]. Similarly active, the Nerine sarniensis (L.) Herb. (Amaryllidaceae) bulb ethyl acetate extract demonstrated toxicity against $\mathrm{L} 1$ larvae $\left(\mathrm{LC}_{50} 8.0 \mu \mathrm{g} / \mathrm{mL}\right.$ ) and adult females (LC $504.6 \mu \mathrm{g} / \mathrm{mosquito}$ ) [104].

Purified fractions of a $n$-hexane extract of Limonia acidissima L. (Rutaceae) leaves showed interesting ovicidal, larvicidal and pupicidal activities. The $\mathrm{LC}_{50}$ for L3 larvae ranged from 4.11 to $23.53 \mu \mathrm{g} / \mathrm{mL}$; for pupae 4.19 to $39.48 \mu \mathrm{g} / \mathrm{mL}$, and the maximum inhibition of egg hatching was $78.4 \%$ at $10 \mathrm{ppm}$. Bioguided fractionation resulted in the isolation of nilocetin (88) [99].

The biological activity of each plant species extract is specific to the plant part(s) and the polarity of the extraction solvent used. Furthermore, activity can differ significantly for the 4 different larval stages $[58,86,89,92-94,96,98,100,108,113,115]$. This variation is discussed below.

A Jatropha curcas L. (Euphorbiaceae) leaves petroleum ether extract showed $\mathrm{LC}_{50} 8.79 \mu \mathrm{g} / \mathrm{mL}$ whereas the root ethanolic extract demonstrated less activity $\left(\mathrm{LC}_{50} 44.75 \mu \mathrm{g} / \mathrm{mL}\right.$ ) [92,98]. A methanolic extract of Gardenia ternifolia Schumach. \& Thonn. (Rubiaceae) leaves was very active $\left(\mathrm{LC}_{50} 32.01 \mu \mathrm{g} / \mathrm{mL}\right.$ ) whereas an acetone extract exhibited lower activity $\left(\mathrm{LC}_{50} 83.31 \mu \mathrm{g} / \mathrm{mL}\right)$ [94]. This study exemplifies the effect of different solvents on the toxicity of the same plant part.

$n$-Hexane, chloroform and acetone extracts of $A$. nilotica seed pods showed weak activity $\left(\mathrm{LC}_{50}\right.$ $103-169 \mu \mathrm{g} / \mathrm{mL}$ ), while ethyl acetate was more active ( $\mathrm{LC}_{50} 59.12 \mu \mathrm{g} / \mathrm{mL}$ ). Interestingly, a benzene extract demonstrated the most activity ( $\mathrm{LC}_{50} 45.32 \mu \mathrm{g} / \mathrm{mL}$ ) [58]. A Ficus benghalensis L. (Moraceae) leaves methanolic extract showed activity against L2, L3 and L4 larvae, but the activity was low for benzene and acetone extracts [93]. Another example of solvent variation was an aqueous extract of Solanum nigrum L. (Solanaceae) fruits which displayed very low activity $\left(\mathrm{LC}_{50} 359.0 \mu \mathrm{g} / \mathrm{mL}\right.$ ) against L3/L4 larvae, while higher activity was observed for a hexanic extract $\left(\mathrm{LC}_{50} 17.63 \mu \mathrm{g} / \mathrm{mL}\right)$ [113].

With not so discrepant, but nonetheless different results, a Pemphis acidula J.R. Forst. \& G. Forst. (Lythraceae) leaves methanolic extract $\left(\mathrm{LC}_{50} 22.10 \mu \mathrm{g} / \mathrm{mL}\right.$ ) was more active than a benzenic leaves extract ( $\mathrm{LC}_{50} 43.99 \mu \mathrm{g} / \mathrm{mL}$ ) [108], while a Tagetes patula L. (Asteraceae) seeds acetone extract ( $\mathrm{LC}_{50} 15.74 \mu \mathrm{g} / \mathrm{mL}$ ) was more active than the $50 \%$ ethanol extract ( $\mathrm{LC}_{50} 25.46 \mu \mathrm{g} / \mathrm{mL}$ ) [93]. P. acidula extracts also demonstrated complete inhibition of egg hatchability at $500 \mathrm{ppm}$ acetone and $450 \mathrm{ppm}$ methanolic [108].

In addition, different parts of Lonchocarpus urucu Killip \& A.C. Sm. (Fabaceae) extracted with the same solvent (methanol) showed different toxicity. The root bark extract was more active ( $\mathrm{LC}_{50}$ $17.6 \mu \mathrm{g} / \mathrm{mL}$ ) than the root medulla extract $\left(\mathrm{LC}_{50} 33.32 \mu \mathrm{g} / \mathrm{mL}\right)$ against L4 larvae [100]. The toxicity of Heracleum rigens Wall. (Apiaceae) seed extracts was evaluated against different larval stages ( $\mathrm{LC}_{50}$ 40.64 to $308.65 \mu \mathrm{g} / \mathrm{mL}$ ), with the petroleum ether extract the most toxic to all larval stages and acetone the least toxic [96]. 
Different organic solvent extracts of Cassia fistula L. (Fabaceae) leaves were evaluated against the mosquito (larvicide, ovicide and repellent). The methanolic extract was the most active for all activities, notably as a larvicidal ( $\left.\mathrm{LC}_{50} 10.69 \mu \mathrm{g} / \mathrm{mL}\right)$. Other extracts also demonstrated high activity against larvae: benzene ( $\left.\mathrm{LC}_{50} 18.27 \mu \mathrm{g} / \mathrm{mL}\right)$ and acetone ( $\left.\mathrm{LC}_{50} 23.95 \mu \mathrm{g} / \mathrm{mL}\right)$. The non-hatching concentration for eggs ranged from 120 to $160 \mathrm{mg} / \mathrm{L}$ and the repellent action $\left(100 \%\right.$ at $\left.5 \mathrm{mg} / \mathrm{cm}^{2}\right)$ ranged from 6.0 to $4.3 \mathrm{~h} \mathrm{[86].}$

Dalbergia brasiliensis Vogel (Fabaceae), commonly known as Jacarandá-da-Bahia in Brazil, is a tree native to the states of Bahia, Minas Gerais, Espírito Santo, Rio de Janeiro and São Paulo. Larvicidal activity of its leaves and trunk bark ethanolic extracts, together with fractions purified by partitioning with $n$-hexane, ethyl acetate and chloroform, were similar $\left(\mathrm{LC}_{50}\right.$ between 24.0 and $\left.44.0 \mu \mathrm{g} / \mathrm{mL}\right)$ [89].

Studies using the incorporation of inorganic nanoparticles, such as zinc oxide and silver in plant extracts, have shown an increase in their biological activity. They are generally easy to obtain, inexpensive, not to mention non-toxic to humans and animals [53]. All of the plant extracts described below showed higher larvicidal activity when incorporated into nanoparticles [53,82,116].

An aqueous extract of Artemisia herba-alba Asso (Asteraceae) leaves was tested against L4 larvae strains from India and Saudi Arabia. The $\mathrm{LC}_{50}$ values were $117.18 \mu \mathrm{g} / \mathrm{mL}$ and $614.54 \mu \mathrm{g} / \mathrm{mL}$ for India and Saudi Arabia larvae, respectively. When the extract was incorporated into silver nanoparticles the activity increased significantly to $10.70 \mu \mathrm{g} / \mathrm{mL}$ and $33.58 \mu \mathrm{g} / \mathrm{mL}$, respectively. Similar results were observed against adult mosquitoes [82].

Aqueous extracts of Ventilago madraspatana Gaertn. (Rhamnaceae) and Zeuxine gracilis (Berda) Bl. (Orchidaceae) leaves were also more toxic to egg, larvae and adult mosquitoes when incorporated into silver nano particle. No egg hatching was observed at $120 \mu \mathrm{g} / \mathrm{mL}$ and $12 \mu \mathrm{g} / \mathrm{mL}$, respectively. Corresponding larvicidal ( $\mathrm{LC}_{50} 26.92 \mu \mathrm{g} / \mathrm{mL}$ and $10.39 \mu \mathrm{g} / \mathrm{mL}$ ) and adulticidal effective dose activities $(44.85 \mu \mathrm{g} / \mathrm{mL}$ and $27.90 \mu \mathrm{g} / \mathrm{mL})$ were observed [112,116].

The activity of a zinc oxide nanoparticle incorporating a Myristica fragans leaf methanolic extract was compared with the crude extract. The activity of the crude extract against the 4 larvae stages ( $\mathrm{LC}_{50} 162.03$ to $273.9 \mu \mathrm{g} / \mathrm{mL}$ ) was less than the nanoparticles ( $\mathrm{LC}_{50} 3.44$ to $10.28 \mu \mathrm{g} / \mathrm{mL}$ ). Similar activity was reported against the pupa (crude extract $\mathrm{LC}_{50} 359.08 \mu \mathrm{g} / \mathrm{mL}$ and nanoparticles $\mathrm{LC}_{50} 14.63 \mu \mathrm{g} / \mathrm{mL}$ ), and female adult forms (crude extract $\mathrm{LC}_{50} 180.26 \mu \mathrm{g} / \mathrm{mosquito}$ and nanoparticles LC $_{50} 15 \mu \mathrm{g} / \mathrm{mosquito)}$ [53].

Following are the results of plant species that demonstrated at least one active extract for larvicidal action. A 90\% ethanol extract of the leaves of Scoparia dulcis L. (Plantaginaceae), a plant used in Brazilian indigenous medicine, demonstrated activity against $\mathrm{L} 4$ larvae $\left(\mathrm{LC}_{50} 83.43 \mu \mathrm{g} / \mathrm{mL}\right)$. The compounds isolated were betulinic acid (22); scopadulcic acid A (23); scopadulcic acid B (24); scopadiol (25); scopadulciol (26) and scopadulin (27) [95].

A methanolic extract of Cinnamosma fragrans Baill. (Canellaceae) root bark demonstrated potential as an insecticide acting in different ways: L1 larvae ( $\left.\mathrm{LC}_{50} 52.5 \mu \mathrm{g} / \mathrm{mL}\right)$, adult $(0.17 \mu \mathrm{g} / \mathrm{mg})$ and $80 \%$ repellency at $20.8 \mu \mathrm{g} / \mathrm{cm}^{2}$ [87]. Similarly, extracts of Ervatamia coronaria (Jacq.) Stapf. (Apocynaceae), commonly known as Capre jasmine, were evaluated for different insecticidal applications. L3 larvicides: benzene ( $\left.\mathrm{LC}_{50} 89.59 \mu \mathrm{g} / \mathrm{mL}\right)$ and ethyl acetate ( $\left.\mathrm{LC}_{50} 97.53 \mu \mathrm{g} / \mathrm{mL}\right)$ [85]. Ovicide/no hatchability: methanol (200 ppm), benzene (250 ppm) and ethyl acetate $(300 \mathrm{ppm})[85,117]$. The repellent activity was the same for all extracts $\left(100 \%\right.$ repellency at $\left.5 \mathrm{mg} / \mathrm{cm}^{2}\right)[85,117]$.

Different extracts were obtained from Mirabilis jalapa L. (Nyctaginaceae) leaves and investigated for their L3 larvicidal activity: methanol ( $\left.\mathrm{LC}_{50} 64.58 \mu \mathrm{g} / \mathrm{mL}\right)$, ethyl acetate $\left(\mathrm{LC}_{50} 72.77 \mu \mathrm{g} / \mathrm{mL}\right)$, chloroform $\left(\mathrm{LC}_{50} 88.20 \mu \mathrm{g} / \mathrm{mL}\right.$ ) and benzene ( $\mathrm{LC}_{50} 97.03 \mu \mathrm{g} / \mathrm{mL}$ ) [103]. Extracts of three other species obtained from the same plant part with different organic solvents were also evaluated. The methanolic extracts were

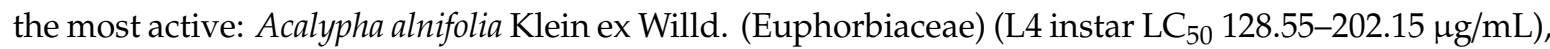
Orthosiphon thymiflorus (Roth) Sleesen (Labiatae) (L3 instar LC 50 149.96-228.13 $\mu \mathrm{g} / \mathrm{mL}$ ) and Ocimum sanctum L. (Labiatae) (L4 instar LC 50 175.67-425.94 $\mu \mathrm{g} / \mathrm{mL}$ ) [80,105,107]. 
Petroleum ether leaf extracts of Pedilanthus tithymaloides (L.) Poit. (Euphorbiaceae), Citrullus colocynthis (L.) Schrad. (Cucurbitaceae) and Phyllanthus amarus Schumach. \& Thonn. (Euphorbiaceae) were active against L4 instar, with $\mathrm{LC}_{50} 55.26 \mu \mathrm{g} / \mathrm{mL}, 74.57 \mu \mathrm{g} / \mathrm{mL}$ and $90.92 \mu \mathrm{g} / \mathrm{mL}$, respectively [88,92]. Similar extracts of Catharanthus roseus (L.) G. Don (Apocynaceae), Eupatorium odoratum L. (Asteraceae), Nyctanthes arbor-tristis L. (Oleaceae), Boenninghausenia albiflora (Hook.) Rchb. ex Meisn. (Rutaceae) and Valeriana hardwickii Wall. (Valerianaceae) presented weak activity $\left(\mathrm{LC}_{50}>100 \mu \mathrm{g} / \mathrm{mL}\right)$ [83].

Larvicidal activity was also reported for Maytenus oblongata Reissek (Celasteraceae) bark ethyl acetate extract $\left(\mathrm{LC}_{50} 74.4 \mu \mathrm{g} / \mathrm{mL}\right.$ ) and Millettia pachycarpa Benth. (Fabaceae) root ethanol extract $\left(\mathrm{LC}_{50} 98.47 \mu \mathrm{g} / \mathrm{mL}\right)$ [101,102]. The latter also demonstrated ovicidal activity at $200 \mathrm{ppm}(100 \%$ non-hatchability) [102].

Ethanol, $n$-hexane and n-butanol extracts of flowers and aerial parts of Buddleja polystachya Fresen. (Buddlejaceae) were evaluated for adulticidal activity at $5 \mu \mathrm{g} / \mathrm{mosquito}$ : the most active was $n$-hexane aerial parts (100\% mortality), followed by $n$-hexane flowers $(96.7 \%$ mortality), ethanol aerial parts $(90 \%$ mortality) and ethanol flowers (83.3\% mortality). Only the n-butanol flower extract was investigated for L1 larvicidal activity, demonstrating $100 \%$ mortality at $1 \mu \mathrm{g} / \mu \mathrm{L}$ [84].

Finally, the species that presented poor larvicidal action for all tested extracts. Aristolochia bracteata Retz. (Aristolochiaceae) leaves methanolic extract was evaluated against L3 larvae (LC $50114.89 \mu \mathrm{g} / \mathrm{mL})$, egg (100\% non-hatchability at $240 \mathrm{ppm})$ and adult mosquito $\left(100 \%\right.$ repellency at $\left.6 \mathrm{mg} / \mathrm{cm}^{2}\right)$ [81]. Similarly, Caesalpinia pulcherrima (L.) Sw. (Fabaceae) extracts were evaluated against larvae: benzene $\left(\mathrm{LC}_{50} 136.37 \mu \mathrm{g} / \mathrm{mL}\right)$ and ethyl acetate $\left(\mathrm{LC}_{50} 144.67 \mu \mathrm{g} / \mathrm{mL}\right)$, and 100\% non-hatchability for benzene (375 ppm) and ethyl acetate (450 ppm). However, both extracts demonstrated 100\% repellency at $5 \mathrm{mg} / \mathrm{cm}^{2}$ [85].

In another study involving a methanol extract of C. pulcherrima, complete inhibition of egg hatching was reported at $300 \mathrm{ppm}$. The repellency was the same as the aforementioned study $\left(5 \mathrm{mg} / \mathrm{cm}^{2}\right)$ [117]. Coccinia indica Wight \& Arn. (Cucurbitaceae) presented similar insecticidal properties for different extracts, with a methanolic extract the most active in terms of ovicidal activity (zero hatchability at $200 \mathrm{ppm})$ and a hexanic extract having the more effective repellency (100\% of repellency at $\left.1 \mathrm{mg} / \mathrm{cm}^{2}\right)$. For 100\% non-hatchability, the concentrations were between 200 ppm and 300 ppm and for $100 \%$ of repellency were between 1 and $5 \mathrm{mg} / \mathrm{cm}^{2}$ [119].

The methanolic extract of Eclipta alba (L.) Hassk (Asteraceae) leaves was also the most active among the solvents of different polarities used to evaluate larvicidal and ovicidal activities of this plant. The $\mathrm{LC}_{50}$ values against L3 larvae were between 127 and $165 \mu \mathrm{g} / \mathrm{mL}$. Complete inhibition of egg hatching occurred at $300 \mathrm{ppm}$ for the methanolic extract and $350 \mathrm{ppm}$ for the other solvents [91].

The methanolic extract of Mentha piperita L. (Lamiaceae) and different extracts of Cardiospermum halicacabum L. (Sapindaceae) showed repellent activity [118,120]. Essential oils and aqueous extracts of the red and pink flowers of $A$. purpurata were investigated for both larvicidal activity and oviposition effect. Similar to the essential oils, the extract of the pink flower was more active than the red, and both disrupted oviposition [67].

In general, organic extracts from different parts of Parthenium hysterophorus (Asteraceae), Pithecellobium dulce (Roxb.) Benth. (Fabaceae) and Solanum xanthocarpum Schrad. \& J.C. Wendl. (Solanaceae) showed weak insecticidal action, requiring high concentrations to demonstrate some biological activity [111,114,121]. Other species that were inactive were Helicteres velutina K. Schum. (Malvaceae), Momordica charantia L. (Cucurbitaceae), Ormosia arborea Vell (Fabaceae), Solanum variabile Mart. (Solanaceae), Spermacoce latifolia Aubl. (Rubiaceae) and Turnera ulmifolia L. (Turneraceae) $[88,95,106]$.

Several organic extracts have shown important insecticidal activities against the Aedes mosquito, including E. transiliensis, E. tirucalli, H. japonicum, N. sarnisiensis, P. longum, P. ribesoides and $P$. sarmentosum. Studies in this section show the potential of plant natural products as insecticides targeting different stages of the mosquito life cycle and how different formulation approaches, such as the incorporation of botanical extracts into silver and zinc nanoparticles, can increase the insecticidal 
effects. Prominent examples include nanoformulations of Z. gracilis, M. fragans and A. herba-herba extracts. These data reinforce the large diversity of plants with toxic effect in different life stages of the Aedes aegypti mosquito. However, an important consideration for these materials is the type of extraction solvent employed, such as n-hexane, chloroform, benzene, given their toxicity to humans associated with harmful residues [122].

\section{Secondary Metabolites}

\subsection{Terpenes}

Terpenoids are a very promising target for the development of products of natural origin to be used in the control of the Ae. aegypti mosquito. These compounds were the most identified in the essential oils, extracts and purified fractions, generally having better results against the mosquito, especially in terms of larvicidal activity. Of the terpenes, monoterpenes are the most active and present great possibilities in bioinsecticide applications due to their low toxicity against mammals and non-target organisms [50].

This significant activity against the mosquito can be explained by the hydrophobicity of this class. Terpene toxicity against Ae. aegypti larvae may be associated with their nonpolar property as reported for other insects $[123,124]$. This property increases the ability of the compound to penetrate the hydrophobic larvae cuticle and renders them more toxic to the insect in comparison to polar compounds [123]. The chemical structures of the terpenes tested are shown in Figures 5-7.

Diterpene 7-oxo-8,11,13-cleistanthatrien-3-ol (28), isolated from the dichloromethane extract of Vellozia gigantea N.L. Menezes \& Mello-Silva (Velloziaceae) adventitious roots, caused 100\% larvae mortality at $416.06 \mu \mathrm{M}$ [125]. The adulticidal activity of the diterpene phytol (29) was $\mathrm{LC}_{50}$ $4.23 \mu \mathrm{M} / \mathrm{mosquito}$ [84].

The triterpenoids ursolic acid (30) and betulinic acid (22) showed larvicidal activity with $\mathrm{LC}_{50}$ $245.24 \mu \mathrm{M}$ and $310.83 \mu \mathrm{M}$, respectively. Their corresponding structures are illustrated in Figure 5 . Bioassays of their chemical derivatives, with esterification of the hydroxyl group at the C-3 position, demonstrated less activity, suggesting that the hydroxyl group plays an important role in larvicidal activity [126].

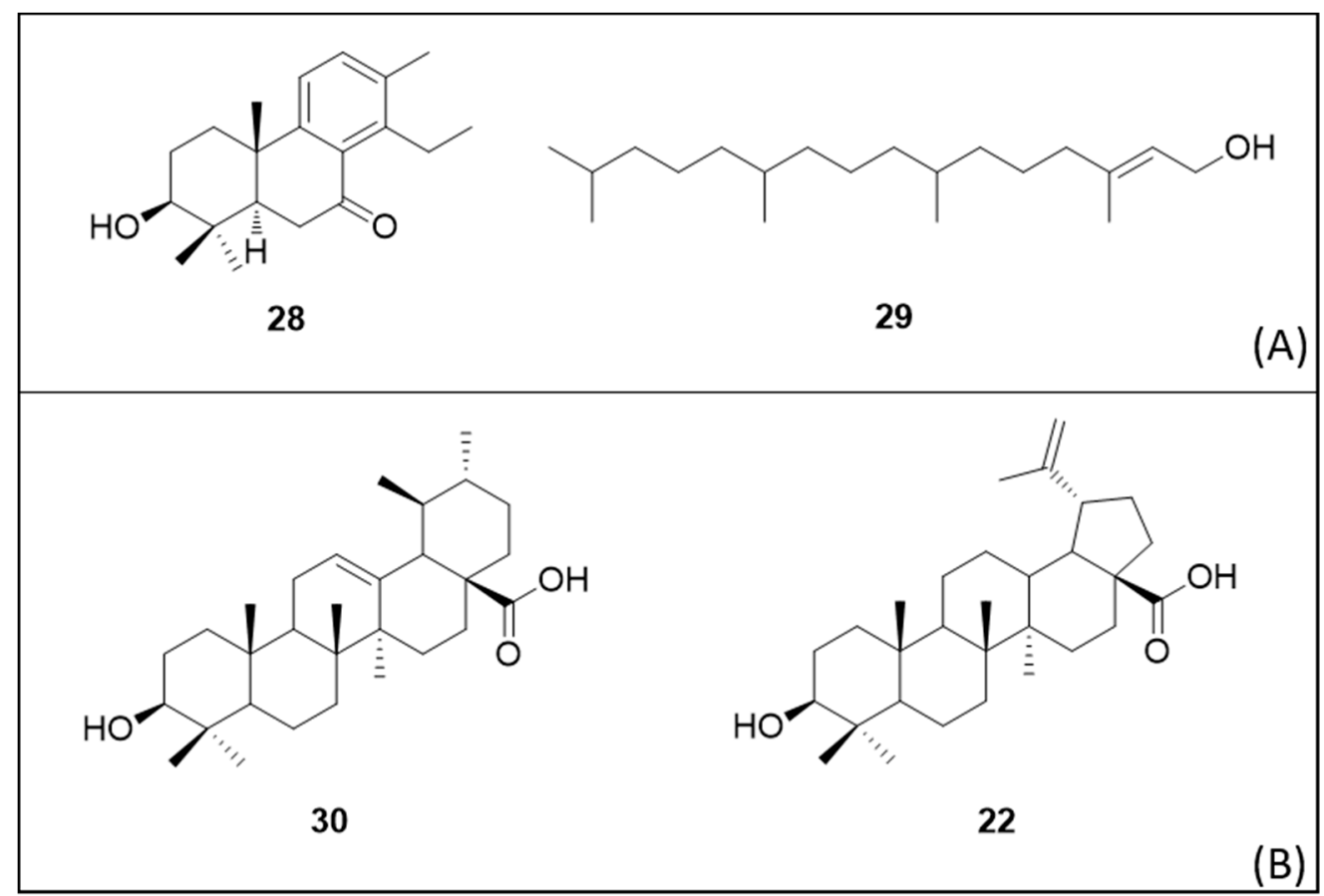

Figure 5. Diterpenes (A) and triterpenes (B) active against Ae. aegypti. 


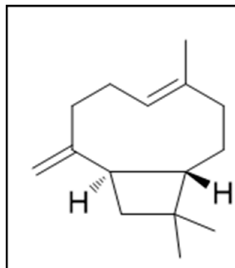

8<smiles>C=C(C(=O)O)[C@H]1CC[C@]2(C)CCC=C(C)[C@H]2C1</smiles>

31<smiles>C=C1C(=O)OC2CC3CCC(=O)/C(C)=C\CC3C1C2</smiles>

32<smiles>C=C1CCCC2(C)OC2CCC2CC(C)(C)CC2C1</smiles>

35

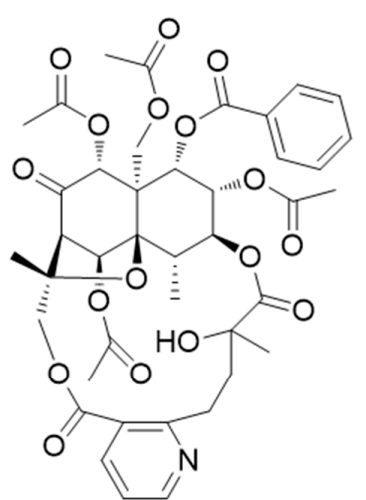

33

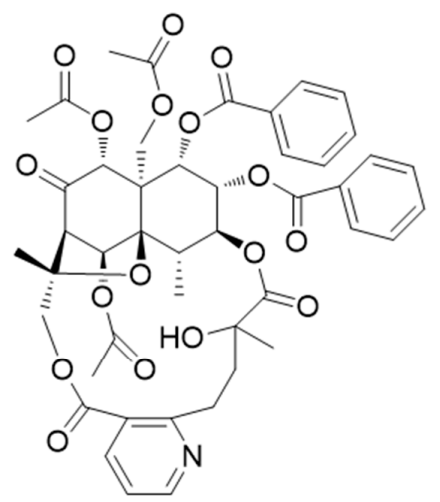

34

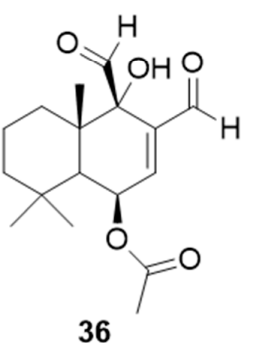

36

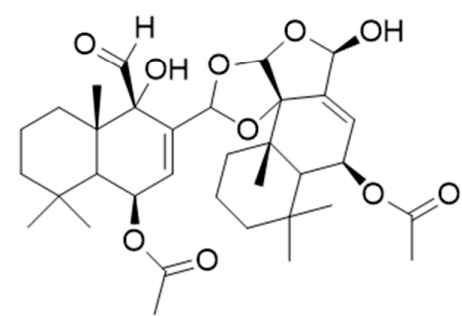

37

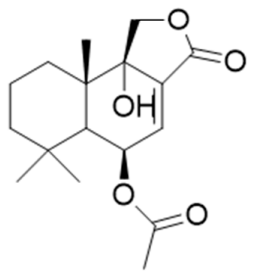

38

Figure 6. Sesquiterpenes active against Ae. aegypti.

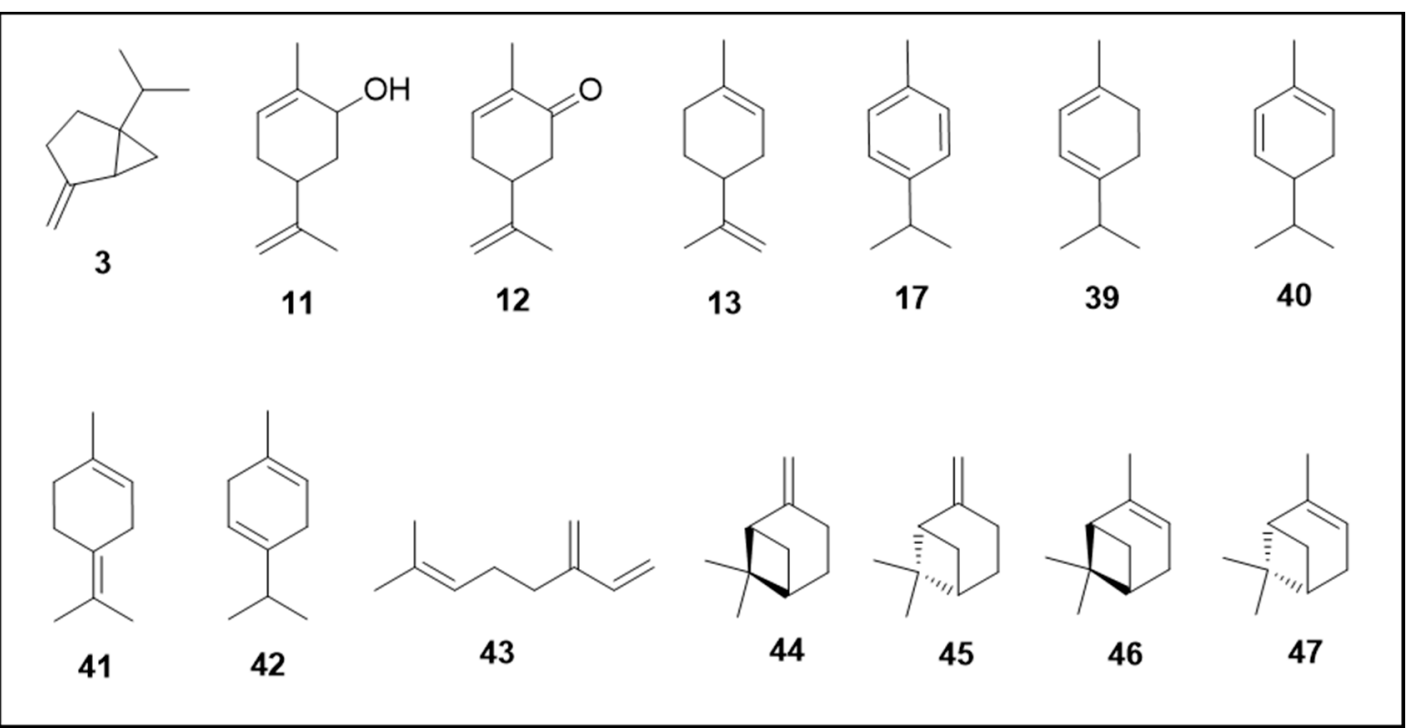

Figure 7. Monoterpenes active against Ae. aegypti. 
The sesquiterpenes $\alpha$-costic acid (31) and inuloxin A (32), both isolated from Inula viscosa (L.) Aiton (Asteraceae), demonstrated strong activity against L1 larvae. The concentration of each terpene required for $100 \%$ mortality was $4.27 \mu \mathrm{M}$ and $4.03 \mu \mathrm{M}$, respectively [127]. Other sesquiterpene alkaloids with strong larvicidal activity (L3 and L4 instar) were 1-O-benzoyl-1-deacetyl-4-deoxyalatamine (33) (LC 50 $9.4 \mu \mathrm{M}$ ) and 1,2-O-dibenzoyl-1,2-deacetyl-4-deoxyalatamine (34) $\left(\mathrm{LC}_{50} 2.3 \mu \mathrm{M}\right)$. These sesquiterpenes with a $\beta$-dihydroagrofuran skeleton were isolated from $M$. oblongata stems [101].

$\beta$-caryophyllene (8) and caryophyllene oxide (35) demonstrated lower larvicidal activity, with $\mathrm{LC}_{50}$ values of 127.23 and $135.24 \mu \mathrm{M}$, respectively [78], together with the sesquiterpene cinnamodial (36), isolated from $C$. fragrans $\left(\mathrm{LC}_{50} 70 \mu \mathrm{M}\right)$ [87]. Regarding adulticidal activity, sesquiterpenes isolated from C. fragrans - cinnamodial (36), cinnafragrin A (37) and cinnamosmolide (38)—showed strong activity with $\mathrm{ED}_{50} 0.29,2.85$ and $12.79 \mathrm{nmol} / \mathrm{mg}$ mosquito, respectively [87].

Monoterpenes were the most evaluated for larvicidal activity with $\mathrm{LC}_{50}$ values ranging from 88 to $540 \mu \mathrm{M}$. The terpene hydrocarbons: limonene (13) $\left(\mathrm{LC}_{50} 88.16 \mu \mathrm{M}\right) ; \alpha$-terpinene (39) $\left(\mathrm{LC}_{50} 107.90 \mu \mathrm{M}\right)$; $\alpha$-phellandrene (40) $\left(\mathrm{LC}_{50} 121.85 \mu \mathrm{M}\right)$, and $\rho$-cymene $(\mathbf{1 7})\left(\mathrm{LC}_{50} 143.05 \mu \mathrm{M}\right)$ were the most active [61,72]. Terpinolene (41), $\gamma$-terpinene (42), $\beta$-myrcene (43) and sabinene (3) showed $\mathrm{LC}_{50} 208.46 \mu \mathrm{M}, 225.35 \mu \mathrm{M}$, $262.78 \mu \mathrm{M}$ and $543.92 \mu \mathrm{M}$, respectively [61,71]. The oxygenated terpene hydrocarbons carvone (11) and cis-carveol (12) demonstrated activity with $\mathrm{LC}_{50}$ of $155.62 \mu \mathrm{M}$ and $218.88 \mu \mathrm{M}$, respectively [72].

A study of the larvicidal activity of $\alpha$-pinene and $\beta$-pinene enantiomers reported the following $\mathrm{LC}_{50}$ values: (-)- $\beta$-pinene (44) $(263.52 \mu \mathrm{M})$ and (+)- $\beta$-pinene (45) $(414.73 \mu \mathrm{M}) ;(-)-\alpha$-pinene (46) $(363.35 \mu \mathrm{M})$ and $(+)-\alpha$-pinene $(47)(484.26 \mu \mathrm{M})$. The (-) enantiomers displayed higher activity than $(+)$, so these results showed that the type of enantiomer and even the racemic mixture could directly interfere with the activity [78].

The monoterpene limonene (13) was incorporated into a nanoemulsion to improve its water solubility and therefore increase its activity [68]. This compound deserves to be highlighted as it presented high oral $\mathrm{LC}_{50}(>4000 \mathrm{mg} / \mathrm{kg})$ and dermatological $\mathrm{LC}_{50}(>5000 \mathrm{mg} / \mathrm{kg})$ values for rodents. It is therefore considered safe and non-toxic to mammals [30].

\subsection{Phenylpropanoids and Phenolic Derivatives}

The chemical structures of the phenylpropanoids and esters discussed in this section are illustrated in Figure 8.

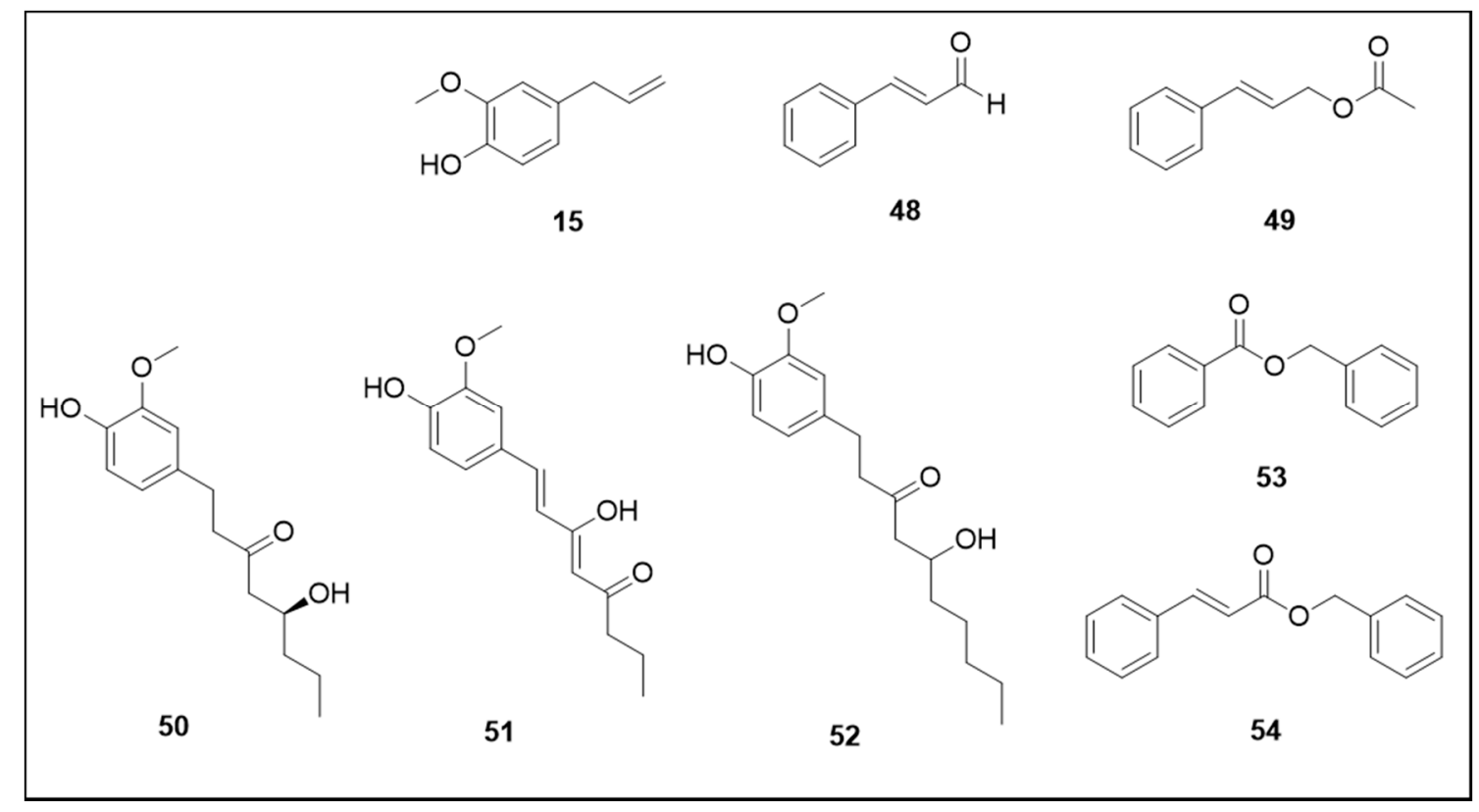

Figure 8. Phenylpropanoids and phenolic derivatives active against Ae. aegypti. 
Among the phenylpropanoids and phenolic derivatives classes cinnamaldehyde (48) and cinnamyl acetate (49) can be highlighted as they presented interesting larvicidal activity (LC 50219.43 and 187.27 $\mu \mathrm{M}$, respectively) [64]. It is important to note that cinnamaldehyde is present in commercial insect-fighting formulations such as Cinamite ${ }^{\circledR}$ and Valero ${ }^{\circledR}$. This information suggests that these secondary metabolites have a high potential for use against Ae. aegypti due to their possible toxicological safety, given that they have been authorized as insecticides since 2001.

Important larvicidal properties were also reported for eugenol (15), a phenolic compound that presents some advantages such as its non-persistence in water and soil, together with its natural degradation in organic acids through the action of Pseudomonas, a soil-dwelling bacterium. Furthermore, it is 1500 times less toxic than pyrethrins and 15,000 times less toxic than azinphos-methyl, an organophosphate [50]. The $\mathrm{LC}_{50}$ value for larvicidal activity was $200.97 \mu \mathrm{M}$ [64]. The phenylpropanoid trans-anethole (7) also showed important action against Ae. aegypti larvae (LC $\mathrm{L}_{50}$ $283.40 \mu \mathrm{M})$ [64].

Metabolites isolated from Zingiber officinale Roscoe (Zingiberaceae) demonstrated strong larvicidal activity, presenting $\mathrm{LC}_{50}$ values of $15.96 \mu \mathrm{M}$ for 4-gingerol (50), $37.36 \mu \mathrm{M}$ for 6-dehydrogingerdione (51) and $61.86 \mu \mathrm{M}$ for 6-gingerol (52) [128]. The phenolic derivatives esters benzyl benzoate (53) and benzyl cinnamate (54)_demonstrated interesting activity as their $100 \%$ larvae mortality concentrations were $117.79 \mu \mathrm{M}$ and $104.92 \mu \mathrm{M}$, respectively [45].

\subsection{Alkaloids and Amides}

The Piperaceae family has numerous compounds with promising activity against Ae. aegypti. The chemical structures of the aforementioned alkaloids and amides are illustrated in Figure 9.

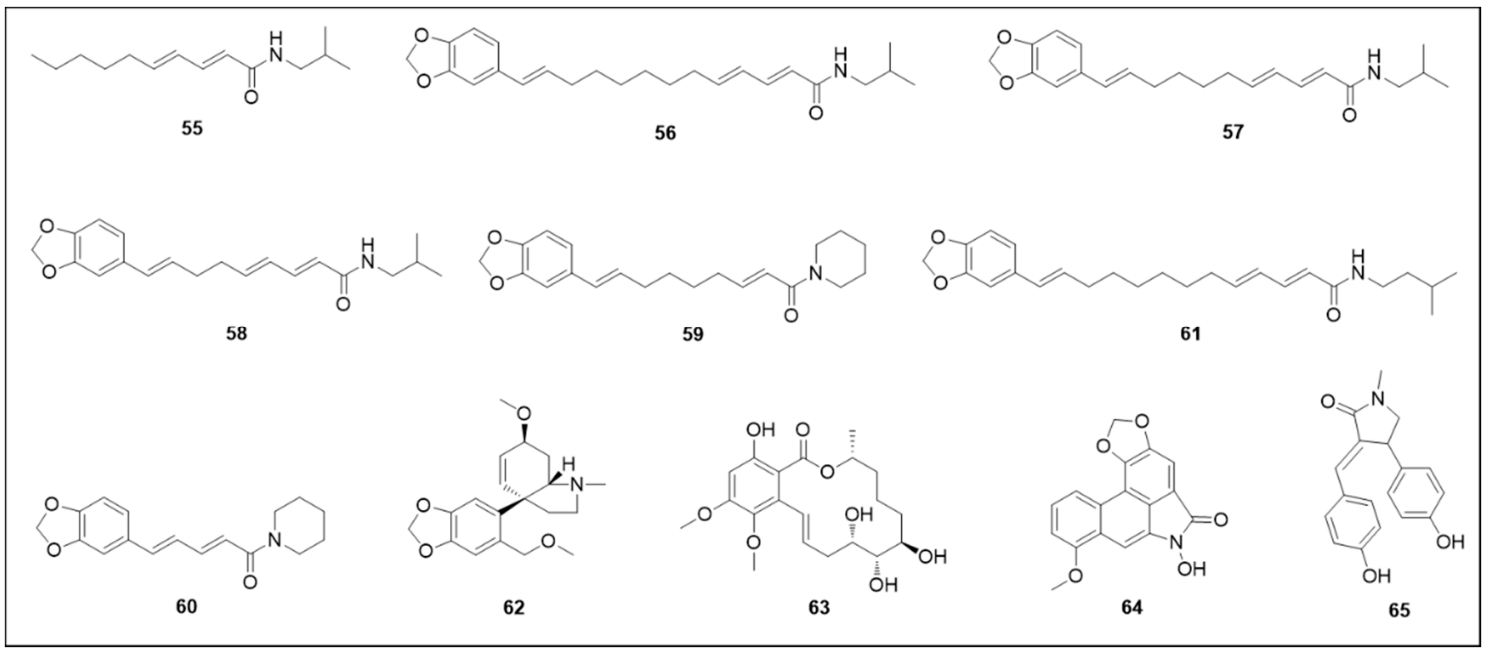

Figure 9. Alkaloids and amides active against Ae. aegypti.

N-Isobutylamide alkaloids from Piper species presented potent larvicidal and adulticidal activities. Secondary metabolites isolated from P. nigrum: pellitorine (55), guineensine (56), pipercide (57) and retrofractamide A (58) presented respective larvicidal $\mathrm{LC}_{50}$ values of $4.12 \mu \mathrm{M}, 2.32 \mu \mathrm{M}, 0.28 \mu \mathrm{M}$ and $0.12 \mu \mathrm{M}$ [129]. Regarding the adulticidal activity, $\mathrm{LC}_{50}$ values ( $\mu \mathrm{M} /$ female mosquito) were 0.76, 4.43, 6.11 and 4.22, respectively [130]. Pipernonaline (59) isolated from the methanolic extract of P. longum, also showed potent larvicidal activity $\left(\mathrm{LC}_{50} 0.73 \mu \mathrm{M}\right)$ [127], while piperine $(60)$ and pipwaqarine $(61)$ isolated from P. nigrum demonstrated $\mathrm{LC}_{50}$ of 17.87 and $75.46 \mu \mathrm{M}$, respectively [131,132].

The analysis of the structure-activity relationship for the N-isobutylamide alkaloids 55-61, it is reasonable to hypothesise that the $\mathrm{N}$-isobutylamine moiety is of crucial importance in terms of larvicidal activity, while the methylenedioxyphenyl moiety does not appear to be essential. 
The mesembrine-type alkaloid sarniensinol (62) isolated from N. sarniensis exhibited strong larvicidal ( $\mathrm{LC}_{50} 24.24 \mu \mathrm{M}$ ) and adulticidal (LC $\mathrm{LC}_{50} 13.88 \mu \mathrm{M} /$ female mosquito) activities [104]. The crinine-type alkaloid crinsarnine (63) only demonstrated strong adulticidal activity $\left(\mathrm{LC}_{50}\right.$ $5.78 \mu \mathrm{M}$ /female mosquito) [133]. N-hydroxyaristolactam I (64), an aristololactam derivative, also showed strong larvicidal activity $\left(\mathrm{LC}_{50} 11.45 \mu \mathrm{M}\right)$ [130], whereas the pyrrolidine alkaloid (Z)-3-(4-hydroxybenzylidene)-4-(4-hydroxyphenyl)-1-methylpyrrolidin-2-one (65) demonstrated weak activity $\left(\mathrm{LC}_{50} 785.86 \mu \mathrm{M}\right)[134,135]$.

\subsection{Thiophenes and Acids}

Thiophene and fatty acid chemical structures are illustrated in Figure 10.

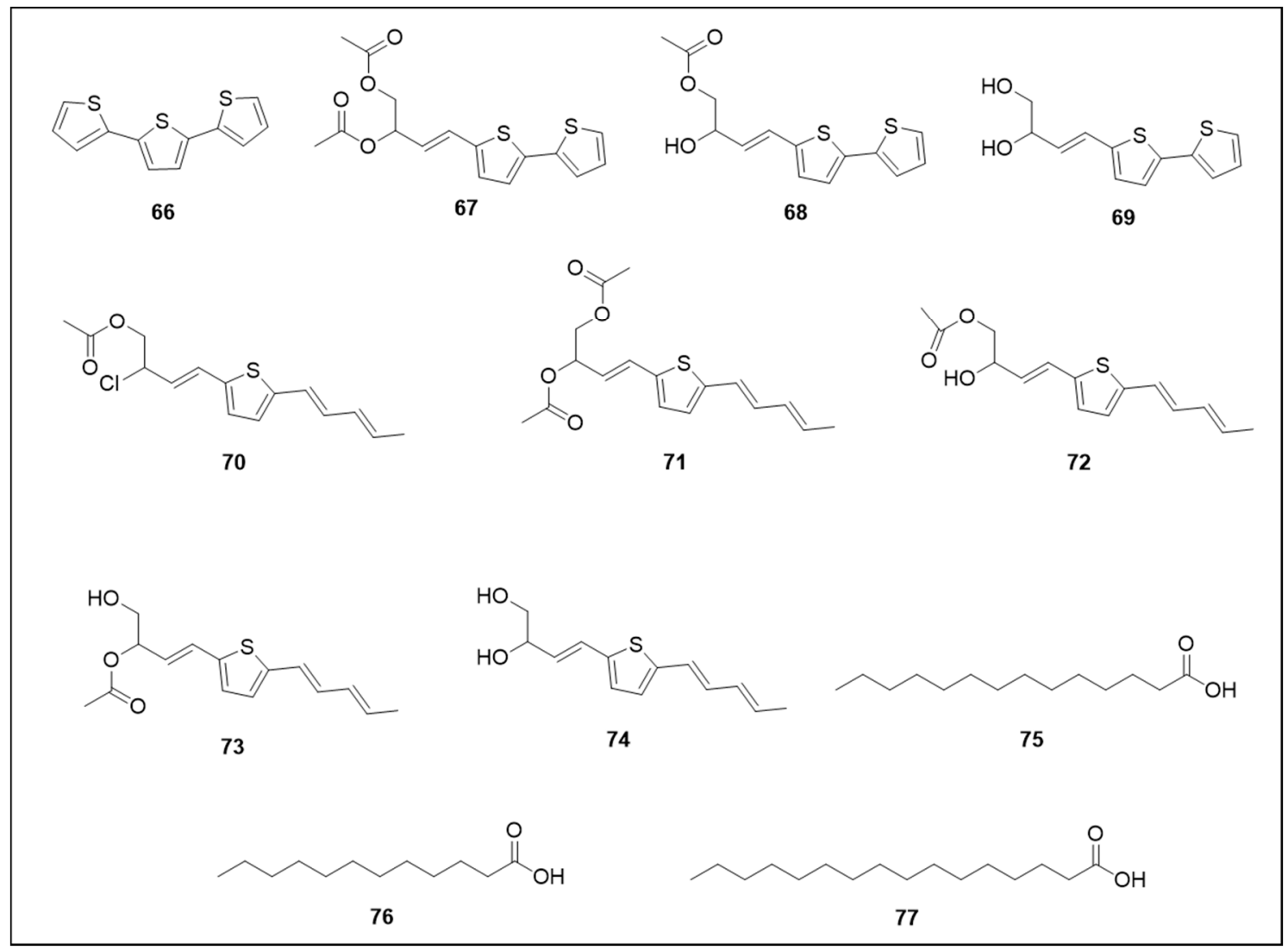

Figure 10. Fatty acids and thiophene derivatives active against Ae. aegypti.

Nine thiophenes, with different numbers of thiophene rings, isolated from E. transiliensis exhibited strong larvicidal activity and a positive correlation was reported between the number of thiophene rings and larvicidal activity, with thiophene derivatives composed of more rings demonstrating more activity [90].

The terthiophene 2,2': $5^{\prime}, 2^{\prime \prime}$-terthiophene (66) was the most active (LC $\left.\mathrm{L}_{50} 0.65 \mu \mathrm{M}\right)$. The activity $\left(\mathrm{LC}_{50}\right)$ of bithiophenes was: 4-(2,2'-bithiophen-5-yl)but-3-yne-1,2-diyl diacetate (67) (12.54 $\mu \mathrm{M})$; 4-(2,2'-bithiophen-5-yl)-2-hydroxybut-3-yn-1-yl acetate (68) (25.31 $\mu \mathrm{M})$ and 4-(2,2' -bithiophen-5-yl)but-3-yne-1,2-diol (69) (39.19 $\mu \mathrm{M})$. Lower larvicidal activity was observed for monothiophenes: 2-chloro-4-[5-(penta-1,3-diyn-1-yl)thiophen-2-yl]but-3-yn-1-yl acetate (70) (49.56 $\mu \mathrm{M}) ; \quad 4$-[5-(penta-1,3-diyn-1-yl)thiophen-2-yl]but-3-yne-1,2-diyl diacetate (71) (56.02 $\mu \mathrm{M}) ; \quad 4-[5-($ penta-1,3-diyn-1-yl)thiophen-2-yl]but-3-yne-1,2-diol (72) (56.68 $\mu \mathrm{MM})$; 2-hydroxy-4-[5-(penta-1,3-diyn-1-yl)thiophen-2-yl]but-3-yn-1-yl acetate (73) (66.64 $\mu \mathrm{M})$ and 1-hydroxy-4-[5-(penta-1,3-diyn-1-yl)thiophen-2-yl]but-3-yn-2-yl acetate (74) (71.74 $\mu \mathrm{M})$ [90]. 
Tetradecanoic acid (75) showed both larvicidal action $\left(\mathrm{LC}_{50} 131.37 \mu \mathrm{M}\right)$ and oviposition attraction $(78.2 \%$ at $43.79 \mu \mathrm{M})[136,137]$. Other fatty acids, hexadecanoic acid (76) and dodecanoic acid (77) displayed lower attraction: $57.4 \%$ for (76) at $3.9 \mu \mathrm{M}$ and $68.8 \%$ for (77) at $249.6 \mu \mathrm{M}$ [137].

\subsection{Flavonoids}

The corresponding chemical structures are shown in Figure 11. Quercetin-4',7-O-dimethyl ether (78), naringenin-7-O-methyl ether (79) and kaempferol-7-O-methyl ether (80) flavonoids isolated from G. ternifolia were active against L2 larvae. The $\mathrm{LC}_{50}$ values were $108.09 \mu \mathrm{M}, 85.37 \mu \mathrm{M}$ and $102.08 \mu \mathrm{M}$, respectively [94].

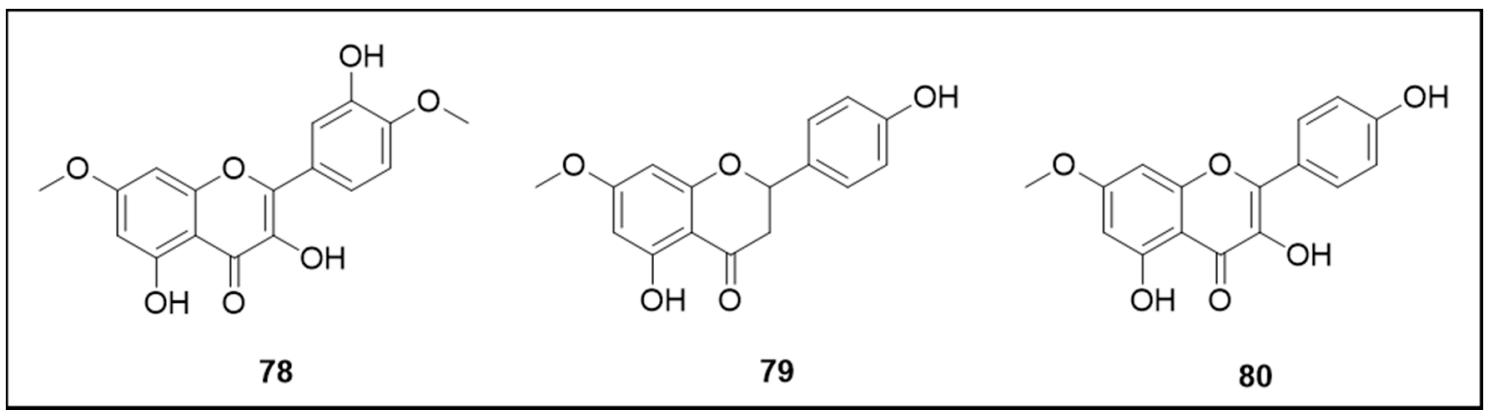

Figure 11. Flavonoids active against Ae. aegypti.

\subsection{Neolignans}

Figure 12 details the neolignan chemical structures.

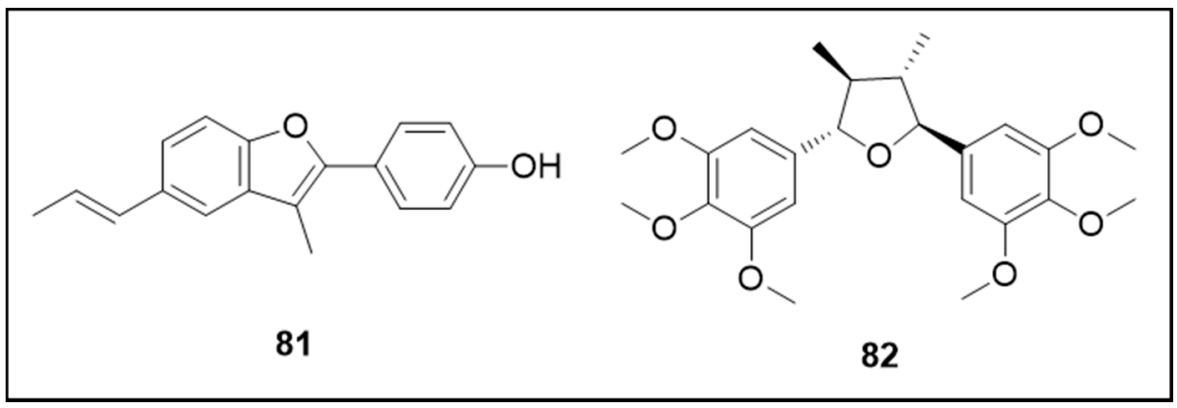

Figure 12. Neolignans active against Ae. aegypti.

Eupomatenoid-6 (81), a neolignan isolated from Piper solmsianum C. DC. (Piperaceae), demonstrated strong larvicidal acitivity $\left(\mathrm{LC}_{50} 19.33 \mu \mathrm{M}\right)$ with probably low toxicity to mammals ( $\mathrm{IC}_{50} 39.30 \mu \mathrm{M}$ for human fibroblast cells, MRC5, with an estimated $\mathrm{LD}_{50}$ of $42.26 \mathrm{mmol} / \mathrm{kg}$ ) [138]. Grandisin (82) presented larvicidal activity $\left(\mathrm{LC}_{50} 346.82 \mu \mathrm{M}\right)$. Histological analysis revealed that this neolignan damages the anterior-middle midgut of the larvae [139].

\subsection{Furanochromones and Furanocoumarin}

The chemical structures of the coumarins are illustrated in Figure 13.

Khellin (83), a natural furanochromone isolated from Ammi visnaga (L.) Lam. (Umbelliferae), caused L3 mortality $\left(\mathrm{LC}_{50} 192.1 \mu \mathrm{M}\right)$ [140]. In another study, 83 demonstrated 100\% larvae mortality at $3.84 \mu \mathrm{M}$ and $75 \%$ adult mortality at $19.21 \mu \mathrm{M} /$ mosquito for permethrin-susceptible strains [141]. Another furanochromone isolated from A. visnaga, visnagin (84) presented $93 \%$ mortality at $4.34 \mu \mathrm{M}$, together with moderate adulticidal activity ( $65 \%$ mortality at $21.72 \mu \mathrm{M} / \mathrm{mosquito})$. In addition, this study investigated 2 furanocoumarins isolated from Ruta graveolens L. Royle (Rutaceae): 5-methoxypsoralen (5-MOP) (85) and 8-methoxypsoralen (8-MOP) (86), which showed moderate adulticidal activity (55\% 
mortality at $23.13 \mu \mathrm{M} /$ mosquito and $67.5 \%$ mortality at $23.13 \mu \mathrm{M} /$ mosquito, respectively). Compound 86 presented weak activity against L3 $(53.3 \%$ mortality at $4.63 \mu \mathrm{M})$ while 85 was inactive [141].

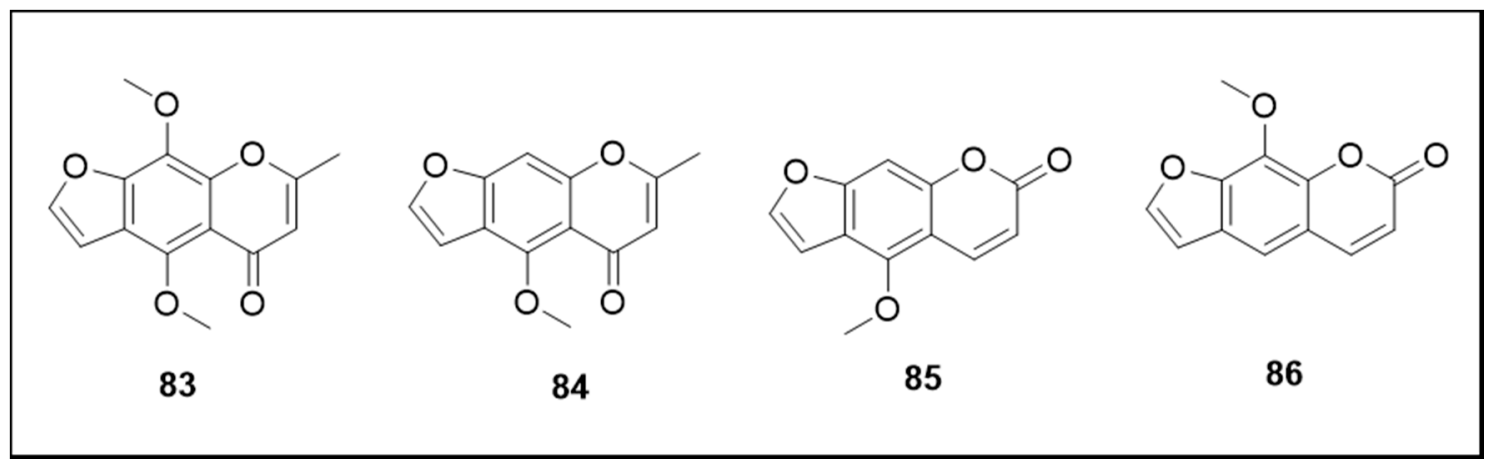

Figure 13. Furanochromones and furanocoumarins active against Ae. aegypti.

\subsection{Other Secondary Metabolites}

The chemical structures of the compounds referenced in this section are illustrated in Figure 14.<smiles>COC1=CC(=O)c2ccccc2C1=O</smiles>

87<smiles>CC(=O)c1ccccc1</smiles>

89<smiles>C[C@H](C[C@H](O)[C@H]1OC1(C)C)[C@H]1CC[C@]2(C)C3=CCC4C(C)(C)C(=O)CC[C@]4(C)C3CC[C@]12C</smiles>

88

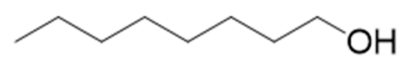

90

Figure 14. Naphthoquinone, protolimonoid, alcohol and ketone with activity against Ae. aegypti.

Several other classes of plant natural products have also been investigated regarding their insecticidal activities. Naphthoquinone 2-methoxy-1,4-naphthoquinone (87) isolated from Impatiens glandulifera (Balsaminaceae) showed extremely potent larvicidal acitivity $\left(\mathrm{LC}_{50} 0.45 \mu \mathrm{M}\right)$ and moderate adulticidal activity $(40 \%$ mortality at $26.6 \mu \mathrm{M})$ [127].

Nilocetin (88), a protolimonoid isolated from L. acidissima, also demonstrated very strong larvicidal $\left(\mathrm{LC}_{50} 0.96 \mu \mathrm{M}\right)$ and pupicidal $\left(\mathrm{LC}_{50} 1.36 \mu \mathrm{M}\right)$ activity which was higher than temephos, a well-documented chemical insecticide. The triterpenoid also caused $83 \%$ egg mortality at 4.38 $\mu \mathrm{M}$ [99]. Already a study evaluating volatile plant metabolites capable of eliciting an Ae. aegypti behavioral response reported that acetophenone (89) attracted adult mosquitoes whereas 1-octanol (90) acted as a repellent (flight aversive response) [142]. 


\section{Mechanisms of Action}

The mechanisms of action for the Ae. aegypti control relate more to the use of conventional chemical insecticides. Table 5 summarizes the mechanisms of action data of the botanical samples discussed in this section. Ae. aegypti control relies primarily on the use of conventional chemical insecticides which target different critical sites in the mosquito life cycle. Organophosphates and carbamates, for example, target acetylcholinesterase enzyme inhibition. Pyrethroids and some organochlorines target sodium channels. Cyclodienes and polychloroterpenes target gamma-aminobutyric acid (GABA) receptors [34].

Other mechanisms of alternative insecticides authorized by regulatory agencies vary in terms of their action. For example, a biological approach employs the use of entomopathogenic bacteria (Bacillus thuringiensis israelensis and Bacillus sphaericus), which act via the toxic action of their spores damaging the intestinal epithelium of larvae. Insect growth regulators (IGR) differ in that they inhibit insect chitin synthesis, and therefore disrupt the moulting process, while juvenile hormone analogs (JHA) act by interfering with the insect's endocrine system [138].

Further studies are required in order to completely understand the various toxic action mechanisms of botanical insecticides. However, a number of mechanisms have been proposed and proven. The majority of mechanism of action studies have focused on the larval stage, particularly feeding and/or contact. In the case of ingestion, the action is usually through digestive toxicity whereas contact may involve enzymatic inhibition, endocrine disruption (acting especially during the moulting process), toxicity to the nervous system and other mechanisms depending on the target site [143].

The rapid toxic action of essential oils against the insect indicates a possible neurotoxic mode of action [144]. Phytochemicals may act in cholinergic, GABA, mitochondrial and octopaminergic systems [145]. A study of five volatile compounds commonly found in plant essential oils—eugenol, geraniol, coumarin, eucalyptol and carvacrol—investigated docking against octopamine and acetylcholinesterase receptors in Ae. aegypti and Homo sapiens protein models. All compounds were found to dock in both protein models, with some more selectivity for insect proteins [146].

Effects on the larval nervous system were observed after treatment with Piper species extracts. Tremor, convulsion, excitement, followed by paralysis and death were verified after exposure of larvae to $P$. longum, $P$. ribesoides and P. sarmentosum extracts. In addition, the larvae showed morphological changes in the anal papillae [109].

Essential oils of I. verum, P. dioica and $M$. fragrans inhibited acetylcholinesterase causing acetylcholine accumulation in the synapses, with the membrane in a constant state of excitement, culminating in ataxia, lack of neuromuscular coordination and eventual death [54]. The neurotoxic effect was also observed for a nanoemulsion with P. emarginatus essential oil. It probably causes reversible inhibition of acetylcholinesterase and consequently larval death [66].

D. brasiliensis extracts caused external morphological alterations in the larvae, resulting in interference in the moulting process. The authors also reported digestive toxicity and morphological changes in the anal papillae and respiratory siphon of the larvae which interfered with swimming and oxygen flow [89]. Similarly, nilocetin (88) (Figure 14) induced morphological deformations together with moulting symptoms and growth disruption in all mosquito life cycle stages. These compounds also totally ruptured the peritrophic membrane [99].

A Lonchocarpus urucu extract caused disruption in the peritrophic matrix, a medium intestine lining composed of chitin and proteins, whose functions are to protect against abrasion caused by food and micro-organisms, among others such as decreasing the excretion of digestive enzymes through their recycling. In addition, this extract caused extensive damage to the midgut epithelium (Table 5) [100]. 
Table 5. Mechanisms of action of botanicals against the Ae. aegypti mosquito.

\begin{tabular}{|c|c|c|c|c|}
\hline Target Site & Mechanism of Action & Compound & Plant Species & Reference \\
\hline \multirow{3}{*}{ Nervous system } & \multirow[t]{2}{*}{ Inhibition of acetylcholinesterase (AChE) } & Essential oil & $\begin{array}{c}\text { Illicium verum, Pimenta dioica and } \\
\text { Myristica fragrans }\end{array}$ & [54] \\
\hline & & Nanoemulsion with essential oil & Pterodon emarginatus & {$[66]$} \\
\hline & Not specified & Ethanolic extract & $\begin{array}{c}\text { Piper longum, Piper ribesoides and Piper } \\
\text { sarmentosum }\end{array}$ & [109] \\
\hline \multirow{2}{*}{ Gut trypsin } & Inhibition of trypsin and consequent decreased & Aqueous extract & Moringa oleifera & [147] \\
\hline & absorption of nutrientes and essential aminoacids & Essential oil & Croton rhamnifolioides & [70] \\
\hline \multirow{3}{*}{ Peritrophic matrix } & Change in internal morphology and consequent insect & Methanol extracts & Derris (Lonchocarpus) urucu & [100] \\
\hline & protection dysfuntion & Nilocetin & Limonia acidissima & [99] \\
\hline & & Methanol extracts & Derris (Lonchocarpus) urucu & [100] \\
\hline \multirow[t]{2}{*}{ Midgut epithelium } & Tissue destruction and cell disorganization & Pellitorine & Asarum heterotropoides & [148] \\
\hline & & Grandisin & Piper solmsianum & [139] \\
\hline \multirow{2}{*}{ Anal papillae } & \multirow{2}{*}{ Morphological changes, interference with the larva swin } & Crude extracts & Dalbergia brasiliensis & [89] \\
\hline & & Ethanolic extract & $\begin{array}{c}\text { Piper longum, Piper ribesoides and Piper } \\
\text { sarmentosum }\end{array}$ & [109] \\
\hline \multirow{2}{*}{ Respiratory siphon } & \multirow{2}{*}{$\begin{array}{c}\text { Morphological changes, interference with the oxygen } \\
\text { flow }\end{array}$} & Crude extracts & Dalbergia brasiliensis & [89] \\
\hline & & Nanoemulsion with limonene & Baccharis reticularia & {$[68]$} \\
\hline \multirow[t]{2}{*}{ Anal gills } & \multirow[t]{2}{*}{ Comprehensive damage; debris in hemolymph } & Pellitorine & Asarum heterotropoides & [148] \\
\hline & & Crude extracts & Dalbergia brasiliensis & [89] \\
\hline \multirow{3}{*}{$\begin{array}{l}\text { Thorax and } \\
\text { exoskeleton }\end{array}$} & \multirow{3}{*}{$\begin{array}{l}\text { Changes in external morphology, interfering with the } \\
\text { molting process }\end{array}$} & Pellitorine & Asarum heterotropoides & [148] \\
\hline & & Nilocetin & Limonia acidissima & [99] \\
\hline & & Nanoemulsion with limonene & Baccharis reticularia & [68] \\
\hline Digestive system & Digestive toxicity & Crude extracts & Dalbergia brasiliensis & [89] \\
\hline
\end{tabular}


Pellitorine (55), an isobutylamide alkaloid, whose structure is illustrated in Figure 9, promoted histological changes in the thorax, midgut and anal gills. These toxic effects probably occur as a result of compound action on the larval osmoregulation system [148]. Already a nanoemulsion with limonene (13) (Figure 7) promoted morphological alterations to the head, siphon, abdomen cuticles and thorax, promoting larvae fragility and low mobility [68].

C. rhamnifolioides essential oil induced toxicity in the larvae by trypsin-like activity. Trypsin is a serine protease that widely occurs in the gut of insects. A decrease in its activity may result in poor nutrient absorption and non-availability of essential aminoacids, causing insect death [72]. A Moringa oleifera (Moringaceae) extract also caused larval toxicity by inhibiting trypsin in the gut [147]. After treatment with grandisin (82) (Figure 12), larvae presented intense tissue destruction and cell disorganization in the anterior midgut [139].

\section{Limitations and/or Expectations of Plant Natural Product Insecticide Applications}

As demonstrated in this review, natural products of botanical origin are promising for control of the Ae. aegypti mosquito, although there remain several limitations and challenges to overcome for their application as insecticidal products. From 1998 until early 2011, the number of patents of essential oil-containing mosquito repellent inventions has almost doubled every 4 years [149], but the number of new products does not reflect this. There are several possible reasons for this disparity, such as: (i) the onerous regulatory processes involved in the registration of a pesticide product; (ii) the quantity of raw material biomass required to obtain sufficient extract and/or its isolated active compound, and (iii) most of the research is conducted at the laboratory scale often without field evaluation to confirm the product application $[13,47,150]$.

The complex process of registering an insecticide discourages companies from investing in new products, especially in some places such as Brazil and the European Union. There are numerous criteria, including provision of non-target toxicology and environmental destination data, extensive data to guarantee plant stability and extract standardization, together with physico-chemical and microbiological procedures establishing quality control of the raw material and final product $[47,48]$.

The low availability of raw materials due to limited yields and cultivation usually makes botanical insecticides more expensive than chemicals. The study of bioactive compound synthesis through biotechnology, such as tissue culture in bioreactors, constitutes an alternative to this limitation [150,151]. In moving from laboratory to industrial scale, a number of different factors must be considered: botanical material, analysis technique, formulations, toxicological tests, mechanisms of action, among others.

Considering the botanical material, it is essential to correctly identify the botanical species and determine the chemical composition of the extract (standardization) $[13,50]$. Chemical composition may vary depending on numerous factors, such as crop period, seasonality, phenological stage, temperature, humidity, luminosity, altitude, pluviometry, ultraviolet radiation, soil and nutrient conditions, geographical locations, collection method, drying and the part of the plant used, among others, and consequently impact insecticidal activity [152-155].

Regarding larvicidal analysis techniques, important considerations are: (i) larval phase, (ii) analysis time, and (iii) the use of positive and negative controls. In different studies, the younger the larval stage, the more susceptible it is to toxic effects, as reported for Ficus benghalensis, Heracleum rigens, Myristica fragans and Solanum xanthocarpum (Table 3) $[53,93,96,114]$. This characteristic may relate to the reduced feeding of larvae in the late L4 instar. If the toxic effect of the insecticide is ingestion-dependent, the effect may be less pronounced the closer the larva is to the pupa stage of metamorphosis [156]. Although most studies use $24 \mathrm{~h}$ as the contact time to express the mortality result, it is important to note that some materials may have delayed activity, actually causing larvae death after 48,72 or even $96 \mathrm{~h}$. Thus, during product development, it is necessary to assess the toxic effect at different time intervals. Finally, the use of negative and positive controls is essential to ensure results reliability, although a number of studies did not report this data [157]. Therefore, test non-uniformity makes it 
difficult to compare the results of different studies. This constitutes another obstacle to overcome for the development of plant natural product insecticides [158].

Understanding the mechanism of action is fundamental in using a material as an inseticidal product. Knowledge of the mechanism of action makes it possible to understand which non-target organisms could be harmed by the use of such products $[145,159]$. In addition, this information facilitates prospecting other possibly more active materials using biotechnology tools and in silico models [160-162]. However, in general it is not easy to understand the mechanisms of action of plant natural products. Normally there are multiple modes of action pertaining to the complex composition of the materials $[30,49,145]$, that usually occur in different target sites, as described for Piper spp, Derris (Lonchocarpus) urucu, Asarum heterotropoides and Dalbergia brasiliensis (Table 5) [89,100,109,148].

During the development stage, it is essential to evaluate toxicity in non-target organisms for promising insecticides using suitable models, such as the fish embryo acute toxicity (FET) test [163]. This model has been proposed to determine the acute or lethal toxicity of materials in the embryonic stages of zebrafish (Danio rerio) and for environmental assessments $[163,164]$. In addition, it is important to consider other aquatic and terrestrial organisms according to the intended application location, such as fish, amphibians, bees, birds and mammals [159]. Considering that natural product insecticides have natural degradation mechanisms, they possibly present advantages in comparison with insecticides of synthetic origin [26,30,50].

In general, raw materials (essential oils, extracts and isolated compounds) from plant natural products are poorly soluble in water and do not persist in the environment, which complicates the application and reduces the effectiveness of the desired action $[26,51,165]$. Therefore, the use of pharmaceutical technology is of fundamental importance in the development of formulations. Among the techniques used, nanotechnology, encapsulation and use of hydrophobic matrices with an extended and controlled release system should be highlighted as they can prolong the residual effect of formulations $[51,149,165-167]$ due to controlled release. Formulation development of natural products also poses a challenge for the application of these materials but it is imperative to improve both efficiency and cost-effectiveness $[150,165]$. Investing in botanical natural product formulations is an important advance in increasing the availability of commercial eco-friendly insecticides for Ae. aegypti control.

\section{Conclusions}

Considering the several stages of the insect development, the larvicidal test is the most evaluated bioassay in the search for insecticides to control Ae. aegypti for a number of reasons: (i) the larval phase is the longest in the immature stage; (ii) larvae are generally more sensitive to the toxic effects of compounds, and (iii) larvae breeding sites are localized and usually accessible. The search for ovicidal action is complex, especially due to its composition that hinders the toxic action of compounds. For the adult phase, there are compounds that cause toxicity by contact as well as those with repellent action.

Some very common edible botanical species such as Petroselinum crispum, Foeniculum vulgare, Curcuma longa, Mentha spicata, Ocimum gratissimium and Rosmarinus officinalis are highlighted, especially in the larval phase of Ae. aegypti, due to their possible low toxicity to non-target organisms. However, other non-edible species have shown strong larvicidal extract activity, among them Echinops transiliensis, Piper ssp, Hypericum japonicum and Nerine sarniensis.

Essential oils provide a promising source for insecticidal applications due to their important insecticidal activities and possible toxicological safety for mammals and the environment. Moreover, they generally possess high oral and dermal $\mathrm{LC}_{50}$ values for these animals and are more readily degraded by natural ecosystem mechanisms.

Among the secondary metabolites, terpenes, especially monoterpenes, and phenylpropanoids are highlighted for larvicidal activity. These compounds are present in large quantities in essential oils. In addition, thiophenes, amides and alkaloids demonstrate high larvicidal and adulticidal activity. 
Regarding the mechanisms of action, botanical natural products extracts, and pure compounds have displayed acitivities that include altering insect morphogenesis and therefore impairing the moulting process, respiration, feeding, and self-defense, among others. In addition, they altered biochemical processes and the nervous system.

Despite the limitations and obstacles to overcome, plant natural products are a suitable alternative source of eco-friendly botanical insecticides to control the Ae. aegypti mosquito, popularly known as dengue mosquito. Ever increasing mosquito resistance to conventional chemical insecticides warrants alternative products, which are safer for the environment and pose less risk to human health.

Author Contributions: M.R.S.S. and P.C.V. contribuited to the design and writing of the manuscript; P.C.V., L.S.E. and N.P.L. supervised and critically revised the final version of the manuscript; All authors have read and agreed to the published version of the manuscript.

Funding: The authors also acknowledge the Ministry of Health and University of Brasilia (TED 74/2016 and TED 42/2017), FAPESP CNPq.

Acknowledgments: The authors acknowledge the Ministry of Health, Conselho Nacional de Desenvolvimento Científico e Tecnológico (CNPq); Coordenação de Aperfeiçoamento de Pessoal de Nível Superior (CAPES; finance code 001) and The Research Network Natural Products against Neglected Diseases (ResNet NPND).

Conflicts of Interest: The authors have no conflict of interest to declare.

\section{References}

1. Consoli, R.A.G.B.; Oliveira, R.L. Principais Mosquitos de Importância Sanitária no Brasil, 1st ed.; Fiocruz: Rio de Janeiro, Brazil, 1994; pp. 115-117.

2. Kraemer, M.U.F.; Sinka, M.E.; Duda, K.A.; Mylne, A.Q.; Shearer, F.M.; Barker, C.M.; Moore, C.G.; Carvalho, R.G.; Coelho, G.E.; Van Bortel, W.; et al. The global distribution of the arbovirus vectors Aedes aegypti and Ae. albopictus. eLife 2015, 4, 08347. [CrossRef]

3. Geris, R.; Ribeiro, P.R.; Brandão, M.D.S.; Da Silva, H.H.G.; Da Silva, I.G. Bioactive Natural Products as Potential Candidates to Control Aedes aegypti, the Vector of Dengue. In Bioactive Natural Products; Elsevier BV: Amsterdam, The Netherlands, 2012; Volume 37, pp. 277-376.

4. Benelli, G.; Jeffries, C.L.; Walker, T. Biological Control of Mosquito Vectors: Past, Present, and Future. Insects 2016, 7, 52. [CrossRef]

5. World Health Organization. Global Strategy for Dengue Prevention and Control. Available online: https://apps.who.int/iris/bitstream/handle/10665/75303/9789241504034_eng.pdf;jsessionid= 59CD5C25000FE7F9ABE29B06C33D6461? sequence=1 (accessed on 27 November 2019).

6. Bhatt, S.; Gething, P.W.; Brady, O.J.; Messina, J.P.; Farlow, A.W.; Moyes, C.L.; Drake, J.M.; Brownstein, J.S.; Hoen, A.G.; Sankoh, O.; et al. The global distribution and burden of dengue. Nature 2013, 496, 504-507. [CrossRef] [PubMed]

7. World Health Organization. Dengue Guidelines for Diagnosis, Treatment, Prevention and Control. Available online: https://apps.who.int/iris/bitstream/handle/10665/44188/9789241547871_eng.pdf?sequence= 1\&isAllowed =y (accessed on 2 December 2019).

8. World Health Organization. Zika Virus: An Epidemiological Update. Available online: https://apps.who.int/ iris/bitstream/handle/10665/255008/WER9215.pdf?sequence=1 (accessed on 5 December 2019).

9. World Health Organization. Chikungunya Disease: Gaps and Opportunities in Public Health and Research in the Americas. Available online: https://www.who.int/wer/2015/wer9042.pdf?ua=1 (accessed on 9 December 2019).

10. Shepard, D.S.; Undurraga, E.A.; Halasa, Y.A.; Stanaway, J.D. The global economic burden of dengue: A systematic analysis. Lancet Infect. Dis. 2016, 16, 935-941. [CrossRef]

11. Teich, V.; Arinelli, R.; Fahham, L. Aedes aegypti e sociedade: O impacto econômico das arboviroses no Brasil. J. Bras. Econ. 2017, 9, 267-276. [CrossRef]

12. Messina, J.; Brady, O.J.; Golding, N.; Kraemer, M.U.F.; Wint, G.R.W.; Ray, S.E.; Pigott, D.M.; Shearer, F.M.; Johnson, K.; Earl, L.; et al. The current and future global distribution and population at risk of dengue. Nat. Microbiol. 2019, 4, 1508-1515. [CrossRef] [PubMed] 
13. Benelli, G. Research in mosquito control: Current challenges for a brighter future. Parasitol. Res. 2015, 114, 2801-2805. [CrossRef]

14. Isman, M.B. A renaissance for botanical insecticides? Pest Manag. Sci. 2015, 71, 1587-1590. [CrossRef]

15. Lees, R.; Gilles, J.R.; Hendrichs, J.; Vreysen, M.J.B.; Bourtzis, K. Back to the future: The sterile insect technique against mosquito disease vectors. Curr. Opin. Insect Sci. 2015, 10, 156-162. [CrossRef]

16. Alphey, L.; McKemey, A.; Nimmo, D.; Oviedo, M.N.; Lacroix, R.; Matzen, K.; Beech, C. Genetic control of Aedes mosquitoes. Pathog. Glob. Heal. 2013, 107, 170-179. [CrossRef]

17. Evans, H.C.; Elliot, S.L.; Barreto, R.W. Entomopathogenic fungi and their potential for the management of Aedes aegypti (Diptera: Culicidae) in the Americas. Mem. Inst. Oswaldo Cruz 2018, 113, 206-214. [CrossRef] [PubMed]

18. Durovni, B.; Saraceni, V.; Eppinghaus, A.; Riback, T.I.S.; Neill, S.L.O.; Moreira, L.A.; Jewell, N.P.; Dufault, S.M.; Simmons, C.P.; Tanamas, S.K.; et al. The impact of large-scale deployment of Wolbachia mosquitoes on arboviral disease incidence in Rio de Janeiro and Niterói, Brazil: Study protocol for a controlled interrupted time series analysis using routine disease surveillance data. F1000 Res. 2019, 8, 1328. [CrossRef]

19. Sarwar, M. Control of dengue carrier Aedes mosquitoes (Diptera: Culicidae) larvae by larvivorous fishes and putting it into practice within water bodies. Int. J. Prev. Med. Res. 2015, 1, 232-237.

20. Cavalcanti, L.P.D.G.; Pontes, R.J.S.; Regazzi, A.C.F.; de Paula Júnior, F.J.; Frutuoso, R.L.; Sousa, E.P.; Dantas Filho, F.D.; Lima, J.W.D.O. Efficacy of fish as predators of Aedes aegypti larvae, under laboratory conditions. Rev. Saude Pública 2007, 41, 638-644. [CrossRef] [PubMed]

21. Nam, V.S.; Yen, N.T.; Duc, H.M.; Tu, T.C.; Thang, V.T.; Le, N.H.; San, L.H.; Loan, L.L.; Huong, V.T.Q.; Khanh, L.H.K.; et al. Community-based control of Aedes aegypti by using Mesocyclops in southern Vietnam. Am. J. Trop. Med. Hyg. 2012, 86, 850-859. [CrossRef] [PubMed]

22. Torres-Estrada, J.L.; Rodríguez, M.H.; Cruz-López, L.; Arredondo-Jimenez, J.I. Selective oviposition by Aedes aegypti (Diptera: Culicidae) in response to Mesocyclops longisetus (Copepoda: Cyclopoidea) under laboratory and field conditions. J. Med. Entomol. 2001, 38, 188-192. [CrossRef] [PubMed]

23. Albeny, D.S.; Martins, G.F.; Andrade, M.R.; Krüger, R.F.; Vilela, E.F. Aedes aegypti survival in the presence of Toxorhynchites violaceus (Diptera: Culicidae) fourth instar larvae. Zoologia 2011, 28, 538-540. [CrossRef]

24. Zuharah, W.F.; Fadzly, N.; Yusof, N.A.; Dieng, H.; Ni, X. Risky behaviors: Effects of Toxorhynchites splendens (Diptera: Culicidae) predator on the behavior of three mosquito species. J. Insect Sci. 2015, 15, 128. [CrossRef]

25. Benelli, G. Plant-borne ovicides in the fight against mosquito vectors of medical and veterinary importance: A systematic review. Parasitol. Res. 2015, 114, 3201-3212. [CrossRef]

26. Pavela, R. Essential oils for the development of eco-friendly mosquito larvicides: A review. Ind. Crops Prod. 2015, 76, 174-187. [CrossRef]

27. Lughadha, E.N.; Govaerts, R.; Belyaeva, I.; Black, N.; Lindon, H.; Allkin, R.; Magill, R.E.; Nicolson, N. Counting counts: Revised estimates of numbers of accepted species of flowering plants, seed plants, vascular plants and land plants with a review of other recent estimates. Phytotaxa 2016, 272, 82-88. [CrossRef]

28. Zebitz, C.P.W. Effects of three different neem seed kernel extracts and azadirachtin on larvae of different mosquito species. J. Appl. Entomol. 1986, 102, 455-463. [CrossRef]

29. Sharma, R.N.; Bhosale, A.S.; Joshi, V.N.; Hebbalkar, D.S.; Tungikar, V.B.; Gupta, A.S.; Patwardhan, S.A. Lavandula gibsonii: A plant with insectistatic potential. Phytoparasitica 1981, 9, 101-109. [CrossRef]

30. Pavela, R. History, presence and perspective of using plant extracts as commercial botanical insecticides and farm products for protection against Insects-A review. Plant Prot. Sci. 2016, 52, 229-241.

31. Yanola, J.; Somboon, P.; Walton, C.; Nachaiwieng, W.; Prapanthadara, L.-a. A novel F1552/C1552 point mutation in the Aedes aegypti voltage-gated sodium channel gene associated with permethrin resistance. Pestic. Biochem. Physiol. 2010, 96, 127-131. [CrossRef]

32. Saavedra-Rodriguez, K.; Urdaneta-Marquez, L.; Rajatileka, S.; Moulton, M.; Flores, A.E.; Fernandez-Salas, I.; Bisset, J.; Rodriguez, M.; Mccall, P.J.; Donnelly, M.J.; et al. A mutation in the voltage-gated sodium channel gene associated with pyrethroid resistance in Latin American Aedes aegypti. Insect Mol. Biol. 2007, 16, 785-798. [CrossRef]

33. Fundação Osvaldo Cruz. Recomendação Técnica Sobre a Interrupção do Uso de Inseticidas Piretróides no Controle do Aedes aegypti no Brasil. Available online: http://www.fiocruz.br/ioc/media/NotaTecnica\%202\% 20IOC\%20v1\%20dengue\%206\%20set\%202011.pdf (accessed on 18 December 2019). 
34. Hemingway, J.; Ranson, H. Insecticide resistance in insect vectors of human diseases. Annu. Rev. Entomol. 2000, 45, 371-391. [CrossRef]

35. Priester, T.M.; Georghiou, G.P. Penetration of permethrin and knockdown in larvae of pyrethroid-resistant and -susceptible strains of the southern house mosquito. J. Econ. Entomol. 1980, 73, 165-167. [CrossRef]

36. Raymond, M.; Chevillon, C.; Guillemaud, T.; Lenormand, T.; Pasteur, N. An overview of the evolution of overproduced esterases in the mosquito Culex pipiens. Philos. Trans. R. Soc. Lond. B 1998, 353, 1707-1711. [CrossRef]

37. Cariño, F.A.; Koener, J.F.; Plapp, F.W.; Feyereisen, R. Constitutive overexpression of the cytochrome P450 gene CYP6A1 in a house fly strain with metabolic resistance to insecticides. Insect Biochem. Mol. Biol. 1994, 24, 411-418. [CrossRef]

38. Mutero, A.; Pralavorio, M.; Bride, J.M.; Fournier, D. Resistance-associated point mutations in insecticideinsensitive acetylcholinesterase. Proc. Natl. Acad. Sci. USA 1994, 91, 5922-5926. [CrossRef] [PubMed]

39. Bisset, J.; Rodríguez, M.M.; Fernández, D. Selection of insensitive acetylcholinesterase as a resistance mechanism in Aedes aegypti (Diptera: Culicidae) from Santiago de Cuba. J. Med. Entomol. 2006, 43, 1185-1189. [CrossRef] [PubMed]

40. Cui, F.; Li, M.X.; Chang, H.J.; Mao, Y.; Zhang, H.Y.; Lu, L.X.; Yan, S.G.; Lang, M.L.; Liu, L.; Qiao, C.L. Carboxylesterase-mediated insecticide resistance: Quantitative increase induces broader metabolic resistance than qualitative change. Pestic. Biochem. Physiol. 2015, 121, 88-96. [CrossRef] [PubMed]

41. Anthony, N.; Rocheleau, T.; Mocelin, G.; Lee, H.J.; Ffrench-Constant, R. Cloning, sequencing and functional expression of an acetylcholinesterase gene from the yellow fever mosquito Aedes aegypti. FEBS Lett. 1995, 368, 461-465. [CrossRef] [PubMed]

42. Lacorte, S.; Ehresmann, N.; Barceló, D. Persistence of temephos and its transformation products in rice crop field waters. Environ. Sci. Technol. 1996, 30, 917-923. [CrossRef]

43. Zhang, A.; Xie, X.; Ye, J.; Lin, C.; Hu, X. Stereoselective toxicity of malathion and its metabolites, malaoxon and isomalathion. Environ. Chem. Lett. 2011, 9, 369-373. [CrossRef]

44. Park, H.-M.; Kim, J.; Chang, K.-S.; Kim, B.-S.; Yang, Y.-J.; Kim, G.-H.; Shin, S.-C.; Park, I.-K. Larvicidal activity of Myrtaceae essential oils and their components against Aedes aegypti, acute toxicity on Daphnia magna, and aqueous residue. J. Med. Entomol. 2011, 48, 405-410. [CrossRef]

45. Seo, S.M.; Park, H.M.; Park, I.K. Larvicidal activity of ajowan (Trachyspermum ammi) and Peru balsam (Myroxylon pereira) oils and blends of their constituents against mosquito, Aedes aegypti, acute toxicity on water flea, Daphnia magna, and aqueous residue. J. Agric. Food Chem. 2012, 60, 5909-5914. [CrossRef]

46. Williams, D.H.; Stone, M.J.; Hauck, P.R.; Rahman, S.K. Why are secondary metabolites (natural products) biosynthesized. J. Nat. Prod. 1989, 52, 1189-1208. [CrossRef]

47. Isman, M.B. Bridging the gap: Moving botanical insecticides from the laboratory to the farm. Ind. Crops Prod. 2017, 110, 10-14. [CrossRef]

48. Miresmailli, S.; Isman, M.B. Botanical insecticides inspired by plant-herbivore chemical interactions. Trends Plant Sci. 2014, 19, 29-35. [CrossRef] [PubMed]

49. Isman, M.B.; Miresmailli, S.; MacHial, C. Commercial opportunities for pesticides based on plant essential oils in agriculture, industry and consumer products. Phytochem. Rev. 2011, 10, 197-204. [CrossRef]

50. Isman, M.B. Plant essential oils for pest and disease management. Crop Prot. 2000, 19, 603-608. [CrossRef]

51. Pavela, R. Encapsulation-A convenient way to extend the persistence of the effect of eco-friendly mosquito larvicides. Curr. Org. Chem. 2016, 20, 2674-2680. [CrossRef]

52. Benelli, G. Plant-mediated biosynthesis of nanoparticles as an emerging tool against mosquitoes of medical and veterinary importance: A review. Parasitol. Res. 2016, 115, 23-34. [CrossRef] [PubMed]

53. Ashokan, A.P.; Paulpandi, M.; Dinesh, D.; Murugan, K.; Vadivalagan, C.; Benelli, G. Toxicity on Dengue mosquito vectors through Myristica fragrans—synthesized zinc oxide nanorods, and their cytotoxic effects on liver cancer cells (HepG2). J. Clust. Sci. 2017, 28, 205-226. [CrossRef]

54. Gomes da Rocha Voris, D.; Dos Santos Dias, L.; Alencar Lima, J.; Dos Santos Cople Lima, K.; Pereira Lima, J.B.; Dos Santos Lima, A.L. Evaluation of larvicidal, adulticidal, and anticholinesterase activities of essential oils of Illicium verum Hook. f., Pimenta dioica (L.) Merr., and Myristica fragrans Houtt. against Zika virus vectors. Environ. Sci. Pollut. Res. 2018, 25, 22541-22551. [CrossRef] [PubMed]

55. Cheng, S.; Chang, H.; Chang, S. Bioactivity of selected plant essential oils against the yellow fever mosquito Aedes aegypti larvae. Bioresour. Technol. 2003, 89, 99-102. [CrossRef] 
56. Kiran, S.R.; Bhavani, K.; Devi, P.S.; Rao, B.R.R.; Reddy, K.J. Composition and larvicidal activity of leaves and stem essential oils of Chloroxylon swietenia DC against Aedes aegypti and Anopheles stephensi. Bioresour. Technol. 2006, 97, 2481-2484. [CrossRef]

57. Komalamisra, N.; Trongtokit, Y.; Rongsriyam, Y.; Apiwathnasorn, C. Screening for lavicidal activity in some Thai plants against four mosquito vector species. Southeast Asian J. Trop. Med. Public Health 2005, 36, 1412-1422.

58. Vivekanandhan, P.; Venkatesan, R.; Ramkumar, G.; Karthi, S.; Senthil-Nathan, S.; Shivakumar, M.S. Comparative analysis of major mosquito vectors response to seed-derived essential oil and seed pod-derived extract from Acacia nilotica. Int. J. Environ. Res. Public Health 2018, 15, 388. [CrossRef] [PubMed]

59. Ali, A.; Akhtar, N.; Khan, B.A.; Khan, M.S.; Rasul, A. Acacia nilotica: A plant of multipurpose medicinal uses. J. Med. Plants Res. 2012, 6, 1492-1496. [CrossRef]

60. Intirach, J.; Junkum, A.; Lumjuan, N.; Chaithong, U.; Jitpakdi, A.; Riyong, D.; Wannasan, A.; Champakaew, D.; Muangmoon, R.; Chansang, A.; et al. Antimosquito property of Petroselinum crispum (Umbellifereae) against the pyrethroid resistant and susceptible strains of Aedes aegypti (Diptera: Culicidae). Environ. Sci. Pollut. Res. 2016, 23, 23994-24008. [CrossRef] [PubMed]

61. Cheng, S.S.; Huang, C.G.; Chen, Y.J.; Yu, J.J.; Chen, W.J.; Chang, S.T. Chemical compositions and larvicidal activities of leaf essential oils from two Eucalyptus species. Bioresour. Technol. 2009, 100, 452-456. [CrossRef]

62. Costa, A.A.; Naspi, C.V.; Lucia, A.; Masuh, H.M.; Barrera, R. Repellent and larvicidal activity of the essential oil from Eucalyptus nitens against Aedes aegypti and Aedes albopictus (Diptera: Culicidae). J. Med. Entomol. 2017, 54, 670-676. [CrossRef]

63. Gillij, Y.G.; Gleiser, R.M.; Zygadlo, J.A. Mosquito repellent activity of essential oils of aromatic plants growing in Argentina. Bioresour. Technol. 2008, 99, 2507-2515. [CrossRef]

64. Cheng, S.-S.; Liu, J.-Y.; Tsai, K.-H.; Chen, W.-J.; Chang, S.-T. Chemical composition and mosquito larvicidal activity of essential oils from leaves of different Cinnamomum osmophloeum provenances. J. Agric. Food Chem. 2004, 52, 4395-4400. [CrossRef]

65. Mendes, L.A.; Martins, G.F.; Valbon, W.R.; da Silva de Souza, T.; Menini, L.; Ferreira, A.; da Silva Ferreira, M.F. Larvicidal effect of essential oils from Brazilian cultivars of Guava on Aedes aegypti L. Ind. Crops Prod. 2017, 108, 684-689. [CrossRef]

66. Oliveira, A.E.; Duarte, J.L.; Amado, J.R.R.; Cruz, R.A.S.; Rocha, C.F.; Souto, R.N.P.; Ferreira, R.M.A.; Santos, K.; da Conceição, E.C.; de Oliveira, L.A.R.; et al. Development of a larvicidal nanoemulsion with Pterodon emarginatus Vogel oil. PLoS ONE 2016, 11, e0145835. [CrossRef]

67. Santos, G.K.N.; Dutra, K.A.; Barros, R.A.; da Câmara, C.A.G.; Lira, D.D.; Gusmão, N.B.; Navarro, D.M.A.F. Essential oils from Alpinia purpurata (Zingiberaceae): Chemical composition, oviposition deterrence, larvicidal and antibacterial activity. Ind. Crops Prod. 2012, 40, 254-260. [CrossRef]

68. Botas, G.D.S.; Cruz, R.A.S.; de Almeida, F.B.; Duarte, J.L.; Araújo, R.S.; Souto, R.N.P.; Ferreira, R.; Carvalho, J.C.T.; Santos, M.G.; Rocha, L.; et al. Baccharis reticularia DC. and limonene nanoemulsions: Promising larvicidal agents for Aedes aegypti (Diptera: Culicidae) control. Molecules 2017, 22, 1990. [CrossRef] [PubMed]

69. Duarte, J.L.; Amado, J.R.R.; Oliveira, A.E.M.F.M.; Cruz, R.A.S.; Ferreira, A.M.; Souto, R.N.P.; Falcão, D.Q.; Carvalho, J.C.T.; Fernandes, C.P. Evaluation of larvicidal activity of a nanoemulsion of Rosmarinus officinalis essential oil. Braz. J. Pharmacog. 2015, 25, 189-192. [CrossRef]

70. Santos, G.K.N.; Dutra, K.A.; Lira, C.S.; Lima, B.N.; Napoleão, T.H.; Paiva, P.M.G.; Maranhão, C.A.; Brandão, S.S.F.; Navarro, D.M.A.F. Effects of Croton rhamnifolioides essential oil on Aedes aegypti oviposition, larval toxicity and trypsin activity. Molecules 2014, 19, 16573-16587. [CrossRef] [PubMed]

71. Cheng, S.S.; Lin, C.Y.; Chung, M.J.; Liu, Y.H.; Huang, C.G.; Chang, S.T. Larvicidal activities of wood and leaf essential oils and ethanolic extracts from Cunninghamia konishii Hayata against the Dengue mosquitoes. Ind. Crops Prod. 2013, 47, 310-315. [CrossRef]

72. Govindarajan, M.; Sivakumar, R.; Rajeswari, M.; Yogalakshmi, K. Chemical composition and larvicidal activity of essential oil from Mentha spicata (Linn.) against three mosquito species. Parasitol. Res. 2012, 110, 2023-2032. [CrossRef]

73. Fayemiwo, K.A.; Adeleke, M.A.; Okoro, O.P.; Awojide, S.H.; Awoniyi, I.O.; Ademolu, K.O. Larvicidal efficacies and chemical composition of essential oils of Pinus sylvestris and Syzygium aromaticum against mosquitoes. Asian Pac. J. Trop. Biomed. 2014, 4, 30-34. [CrossRef] 
74. Oliveira, G.; Cardoso, S.; Junior, C.L. Chemical study and larvicidal activity against Aedes aegypti of essential oil of Piper aduncum L. (Piperaceae). An. Acad. Bras. Cienc. 2013, 85, 1227-1234. [CrossRef]

75. Fontes, U.R., Jr.; Ramos, C.S.; Serafini, M.R.; Cavalcanti, S.C.H.; Alves, P.B.; Lima, G.M.; Andrade, P.H.S.; Bonjardim, L.R.; Quintans, L.J., Jr.; Araujo, A.A.S. Evaluation of the lethality of Porophyllum ruderale essential oil against Biomphalaria glabrata, Aedes aegypti and Artemia salina. Afr. J. Biotechnol. 2012, 11, 3169-3172. [CrossRef]

76. Ali, A.; Tabanca, N.; Kurkcuoglu, M.; Duran, A.; Blythe, E.K.; Khan, I.A.; Baser, K.H.C. Chemical composition, larvicidal, and biting deterrent activity of essential oils of two subspecies of Tanacetum argenteum (Asterales: Asteraceae) and individual constituents against Aedes aegypti (Diptera: Culicidae). J. Med. Entomol. 2014, 51, 824-830. [CrossRef] [PubMed]

77. Chellappandian, M.; Thanigaivel, A.; Vasantha-Srinivasan, P.; Edwin, E.S.; Ponsankar, A.; Selin-Rani, S.; Kalaivani, K.; Senthil-Nathan, S.; Benelli, G. Toxicological effects of Sphaeranthus indicus Linn. (Asteraceae) leaf essential oil against human disease vectors, Culex quinquefasciatus Say and Aedes aegypti Linn., and impacts on a beneficial mosquito predator. Environ. Sci. Pollut. Res. 2018, 25, 10294-10306. [CrossRef]

78. DeSousa, L.M.; de Carvalho, J.L.; Gois, R.W.S.; da Silva, H.C.; Santiago, G.M.P.; Lemos, T.L.G.; Arriaga, A.M.C.; Alves, P.B.; de Matos, I.L.; Militão, G.C.G.; et al. Chemical composition, larvicidal and cytotoxic activities of the essential oils from two Bauhinia species. Rec. Nat. Prod. 2016, 10, 341-348.

79. Kerdudo, A.; Gonnot, V.; Ellong, E.N.; Boyer, L.; Chandre, F.; Adenet, S.; Rochefort, K.; Michel, T.; Fernandez, X. Composition and bioactivity of Pluchea carolinensis (Jack.) G. essential oil from Martinique. Ind. Crops Prod. 2016, 89, 295-302. [CrossRef]

80. Kovendan, K.; Murugan, K.; Vincent, S. Evaluation of larvicidal activity of Acalypha alnifolia Klein ex Willd. (Euphorbiaceae) leaf extract against the malarial vector, Anopheles stephensi, dengue vector, Aedes aegypti and Bancroftian filariasis vector, Culex quinquefasciatus (Diptera: Culicidae). Parasitol. Res. 2012, 110, 571-581. [CrossRef] [PubMed]

81. Krishnappa, K.; Elumalai, K. Toxicity of Aristolochia bracteata methanol leaf extract against selected medically important vector mosquitoes (Diptera: Culicidae). Asian Pacific J. Trop. Dis. 2012, 2, S553-S557. [CrossRef]

82. Aziz, A.T.; Alshehri, M.A.; Panneerselvam, C.; Murugan, K.; Trivedi, S.; Mahyoub, J.A.; Hassan, M.M.; Maggi, F.; Sut, S.; Dall'Acqua, S.; et al. The desert wormwood (Artemisia herba-alba)—From arabian folk medicine to a source of green and effective nanoinsecticides against mosquito vectors. J. Photochem. Photobiol. B Biol. 2018, 180, 225-234. [CrossRef]

83. Alam, M.F.; Safhi, M.M.; Chopra, A.K.; Dua, V.K. Toxicological properties of several medicinal plants from the Himalayas (India) against vectors of malaria, filariasis and dengue. Trop. Biomed. 2011, 28, 343-350.

84. El-Gamal, A.; Al-Massarani, S.; Fawzy, G.; Ati, H.; Al-Rehaily, A.; Basudan, O.; Abdel-Kader, M.; Tabanca, N.; Becnel, J. Chemical composition of Buddleja polystachya aerial parts and its bioactivity against Aedes aegypti. Nat. Prod. Res. 2017, 32, 2775-2782. [CrossRef]

85. Govindarajan, M.; Mathivanan, T.; Elumalai, K.; Krishnappa, K.; Anandan, A. Mosquito larvicidal, ovicidal, and repellent properties of botanical extracts against Anopheles stephensi, Aedes aegypti, and Culex quinquefasciatus (Diptera: Culicidae). Parasitol. Res. 2011, 109, 353-367. [CrossRef]

86. Govindarajan, M. Bioefficacy of Cassia fistula Linn. (Leguminosae) leaf extract against chikungunya vector, Aedes aegypti (Diptera: Culicidae). Eur. Rev. Med. Pharmacol. Sci. 2009, 13, 99-103.

87. Inocente, E.A.; Shaya, M.; Acosta, N.; Rakotondraibe, L.H.; Piermarini, P.M. A natural agonist of mosquito TRPA1 from the medicinal plant Cinnamosma fragrans that is toxic, antifeedant, and repellent to the yellow fever mosquito Aedes aegypti. PLoS Negl. Trop. Dis. 2018, 12, e0006265. [CrossRef]

88. Rahuman, A.A.; Venkatesan, P. Larvicidal efficacy of five Cucurbitaceous plant leaf extracts against mosquito species. Parasitol. Res. 2008, 103, 133-139. [CrossRef]

89. Dalarmi, L.; Silva, C.B.d.; Ocampos, F.M.; Burci, M.L.; Nascimento, K.F.d.; Jesus, C.d.; Dias Gaspari, J.d.F.; Miguel, M.D.; Miguel, O.G.; Zanin, S.M.W. Larvicidal activity of Dalbergia brasiliensis (FabaceaePapilionoideae). Afr. J. Pharm. Pharmacol. 2015, 9, 881-885.

90. Nakano, H.; Ali, A.; Ur Rehman, J.; Mamonov, L.K.; Cantrell, C.L.; Khan, I.A. Toxicity of thiophenes from Echinops transiliensis (Asteraceae) against Aedes aegypti (Diptera: Culicidae) larvae. Chem. Biodivers. 2014, 11, 1001-1009. [CrossRef] 
91. Govindarajan, M.; Karuppannan, P. Mosquito larvicidal and ovicidal properties of Eclipta alba (L.) Hassk (Asteraceae) against chikungunya vector, Aedes aegypti (Linn.) (Diptera: Culicidae). Asian Pac. J. Trop. Med. 2011, 4, 24-28. [CrossRef]

92. Rahuman, A.A.; Gopalakrishnan, G.; Venkatesan, P.; Geetha, K. Larvicidal activity of some Euphorbiaceae plant extracts against Aedes aegypti and Culex quinquefasciatus (Diptera: Culicidae). Parasitol. Res. 2008, 102, 867-873. [CrossRef]

93. Govindarajan, M. Larvicidal efficacy of Ficus benghalensis L. plant leaf extracts against Culex quinquefasciatus Say, Aedes aegypti L. and Anopheles stephensi L. (Diptera: Culicidae). Eur. Rev. Med. Pharmacol. Sci. 2010, 14, 107-111.

94. Ochieng, C.O.; Mid, J.O.; Owu, P.O. Anti-plasmodial and larvicidal effects of surface exudates of Gardenia ternifolia aerial parts. Res. J. Pharmacol. 2010, 4, 45-50. [CrossRef]

95. Dos Santos, E.A.; de Carvalho, C.M.D.; Costa, A.L.S.; Conceição, A.S.; Moura, F.D.B.P.; Santana, A.E.G. Bioactivity evaluation of plant extracts used in indigenous medicine against the snail, Biomphalaria glabrata, and the larvae of Aedes aegypti. Evid. Based Complement. Altern. Med. 2012, 2012, 846583. [CrossRef]

96. Kumuda, S.S.; Mohankumar, T.K.; Prathibha, K.P.; Vijayan, V.A. Efficacy of plant extracts against the larvae of filariasis vector, Culex quinquefasciatus Say and the Dengue vector Aedes aegypti Linn at Mysore. Int. J. Curr. Microbiol. Appl. Sci. 2015, 4, 242-249.

97. Puthur, S.; Anoopkumar, A.N.; Rebello, S.; Aneesh, E.M. Hypericum japonicum: A double-headed sword to combat vector control and cancer. Appl. Biochem. Biotechnol. 2018, 186, 1-11. [CrossRef]

98. Komansilan, A.; Suriani, N.W.; Lawalata, H. Test toxic tuba root extract as a natural insecticide on larvae of Aedes aegypti mosquito vector of dengue fever. Int. J. ChemTech Res. 2017, 10, 522-528.

99. Reegan, A.D.; Gandhi, M.R.; Paulraj, M.G.; Balakrishna, K.; Ignacimuthu, S. Effect of niloticin, a protolimonoid isolated from Limonia acidissima L. (Rutaceae) on the immature stages of dengue vector Aedes aegypti L. (Diptera: Culicidae). Acta Trop. 2014, 139, 67-76. [CrossRef]

100. Gusmão, D.S.; Páscoa, V.; Mathias, L.; Vieira, I.J.C.; Braz-Filho, R.; Lemos, F.J.A. Derris (Lonchocarpus) итиси (Leguminosae) extract modifies the peritrophic matrix structure of Aedes aegypti (Diptera: Culicidae). Mem. Inst. Oswaldo Cruz 2002, 97, 371-375. [CrossRef]

101. Touré, S.; Nirma, C.; Falkowski, M.; Dusfour, I.; Boulogne, I.; Jahn-Oyac, A.; Coke, M.; Azam, D.; Girod, R.; Moriou, C.; et al. Aedes aegypti larvicidal sesquiterpene alkaloids from Maytenus oblongata. J. Nat. Prod. 2017, 80, 384-390. [CrossRef]

102. Lalchhandama, K. Mosquitocidal activity of Millettia pachycarpa on the larvae and eggs of Aedes aegypti. Ann. Biol. Res. 2011, 2, 217-222.

103. Govindarajan, M.; Ramya, A.; Sivakumar, R. Mosquito larvicidal properties of Mirabilis jalapa (Nyctaginaceae) against Anopheles stephensi, Aedes aegypti \& Culex quinquefasciatus (Diptera: Culicidae). Indian J. Med. Res. 2014, 140, 438-440.

104. Masi, M.; van der Westhuyzen, A.E.; Tabanca, N.; Evidente, M.; Cimmino, A.; Green, I.R.; Bernier, U.R.; Becnel, J.J.; Bloomquist, J.R.; van Otterlo, W.A.L.; et al. Sarniensine, a mesembrine-type alkaloid isolated from Nerine sarniensis, an indigenous South African Amaryllidaceae, with larvicidal and adulticidal activities against Aedes aegypti. Fitoterapia 2017, 116, 34-38. [CrossRef]

105. Anees, A.M. Larvicidal activity of Ocimum sanctum Linn. (Labiatae) against Aedes aegypti (L.) and Culex quinquefasciatus (Say). Parasitol. Res. 2008, 103, 1451-1453. [CrossRef]

106. Porto, K.R.d.A.; Motti, P.R.; Yano, M.; Roel, A.R.; Cardoso, C.A.L.; Matias, R. Screening of plant extracts and fractions on Aedes aegypti larvae found in the state of Mato Grosso do Sul (Linnaeus, 1762) (Culicidae). An. Acad. Bras. Cienc. 2017, 89, 895-906. [CrossRef]

107. Kovendan, K.; Murugan, K.; Vincent, S.; Barnard, D.R. Mosquito larvicidal properties of Orthosiphon thymiflorus (Roth) Sleesen. (Family: Labiatae) against mosquito vectors, Anopheles stephensi, Culex quinquefasciatus and Aedes aegypti (Diptera: Culicidae). Asian Pac. J. Trop. Med. 2012, 5, 299-305. [CrossRef]

108. Samidurai, K.; Jebanesan, A.; Saravanakumar, A.; Govindarajan, M.; Pushpanathan, T. Larvicidal, ovicidal and repellent activities of Pemphis acidula Forst. (Lythraceae) against filarial and dengue vector mosquitoes. Acad. J. Entomol. 2009, 2, 62-66.

109. Chaitong, U.; Choochote, W.; Kamsuk, K.; Jitpakdi, A.; Tippawangkosol, P.; Chaiyasit, D.; Champakaew, D.; Tuetun, B.; Pitasawat, B. Larvicidal effect of pepper plants on Aedes aegypti (L.) (Diptera: Culicidae). J. Vector Ecol. 2006, 31, 138-144. [CrossRef] 
110. Santiago, V.S.; Alvero, R.G.; Villaseñor, I.M. Aedes aegypti larvicide from the ethanolic extract of Piper nigrum black peppercorns. Nat. Prod. Res. 2015, 29, 441-443. [CrossRef]

111. Govindarajan, M.; Rajeswary, M.; Sivakumar, R. Larvicidal \& ovicidal efficacy of Pithecellobium dulce (Roxb.) Benth. (Fabaceae) against Anopheles stephensi Liston \& Aedes aegypti Linn. (Diptera: Culicidae). Indian J. Med. Res. 2013, 138, 129-134.

112. Azarudeen, R.M.S.T.; Govindarajan, M.; AlShebly, M.M.; AlQahtani, F.S.; Amsath, A.; Benelli, G. One pot green synthesis of colloidal silver nanocrystals using the Ventilago maderaspatana leaf extract: Acute toxicity on malaria, zika virus and filariasis mosquito vectors. J. Clust. Sci. 2017, 28, 369-392. [CrossRef]

113. Raghavendra, K.; Singh, S.P.; Subbarao, S.K.; Dash, A.P. Laboratory studies on mosquito larvicidal efficacy of aqueous \& hexane extracts of dried fruit of Solanum nigrum Linn. Indian J. Med. Res. 2009, 130, 74-77.

114. Kumar, P.M.; Murugan, K.; Kovendan, K.; Panneerselvam, C.; Kumar, K.P.; Amerasan, D.; Subramaniam, J.; Kalimuthu, K.; Nataraj, T. Mosquitocidal activity of Solanum xanthocarpum fruit extract and copepod Mesocyclops thermocyclopoides for the control of dengue vector Aedes aegypti. Parasitol. Res. 2012, 111, 609-618. [CrossRef]

115. Munhoz, V.M.; Longhini, R.; Souza, J.R.P.; Zequi, J.A.C.; Mello, E.V.S.L.; Lopes, G.C.; Mello, J.C.P. Extraction of flavonoids from Tagetes patula: Process optimization and screening for biological activity. Braz. J. Pharmacog. 2014, 24, 576-583. [CrossRef]

116. Kovendan, K.; Chandramohan, B.; Govindarajan, M.; Jebanesan, A.; Kamalakannan, S.; Vincent, S.; Benelli, G. Orchids as sources of novel nanoinsecticides? Efficacy of Bacillus sphaericus and Zeuxine gracilis—fabricated silver nanoparticles against dengue, malaria and filariasis mosquito vectors. J. Clust. Sci. 2018, 29, 345-357. [CrossRef]

117. Govindarajan, M.; Mathivanan, T.; Elumalai, K.; Krishnappa, K.; Anandan, A. Ovicidal and repellent activities of botanical extracts against Culex quinquefasciatus, Aedes aegypti and Anopheles stephensi (Diptera: Culicidae). Asian Pac. J. Trop. Biomed. 2011, 1, 43-48. [CrossRef]

118. Govindarajan, M.; Sivakumar, R. Repellent properties of Cardiospermum halicacabum Linn. (Family: Sapindaceae) plant leaf extracts against three important vector mosquitoes. Asian Pac. J. Trop. Med. 2012, 2, 602-607. [CrossRef]

119. Govindarajan, M. Ovicidal and repellent properties of Coccinia indica Wight and Arn. (Family: Cucurbitaceae) against three important vector mosquitoes. Eur. Rev. Med. Pharmacol. Sci. 2011, 15, 1010-1019. [PubMed]

120. Bhargava, S. Mosquito repellency potential of the essential oil from Mentha spp. Int. J. Pharm. Pharm. Res. 2016, 6, 509-522.

121. Kumar, S.; Singh, A.P.; Nair, G.; Batra, S.; Seth, A.; Wahab, N.; Warikoo, R. Impact of Parthenium hysterophorus leaf extracts on the fecundity, fertility and behavioural response of Aedes aegypti L. Parasitol. Res. 2011, 108, 853-859. [CrossRef]

122. Dick, F.D. Solvent Neurotoxicity. Occup. Environ. Med. 2006, 63, 221-226. [CrossRef]

123. Vincent, J.F.V.; Wegst, U.G.K. Design and mechanical properties of insect cuticle. Arthropod Struct. Dev. 2004, 33, 187-199. [CrossRef]

124. Fahmy, M.A.H.; Mallipudi, N.M.; Fukuto, T.R. Selective toxicity of N,N'-thiodicarbamates. J. Agric. Food Chem. 1978, 26, 550-557. [CrossRef]

125. Ferreira, M.C.; Cantrell, C.L.; Duke, S.O.; Ali, A.; Rosa, L.H. New pesticidal diterpenoids from Vellozia gigantea (Velloziaceae), an endemic neotropical plant living in the endangered Brazilian biome rupestrian grasslands. Molecules 2017, 22, 175. [CrossRef]

126. Da Silva, G.N.; Trindade, F.T.; Dos Santos, F.; Gosmann, G.; E Silva, A.A.; Gnoatto, S.C. Larvicidal activity of natural and modified triterpenoids against Aedes aegypti (Diptera: Culicidae). Pest Manag. Sci. 2016, 72, 1883-1887. [CrossRef]

127. Masi, M.; Cimmino, A.; Tabanca, N.; Becnel, J.J.; Bloomquist, J.R.; Evidente, A. A survey of bacterial, fungal and plant metabolites against Aedes aegypti (Diptera: Culicidae), the vector of yellow and dengue fevers and zika virus. Open Chem. 2017, 15, 156-166. [CrossRef]

128. Rahuman, A.A.; Gopalakrishnan, G.; Venkatesan, P.; Geetha, K.; Bagavan, A. Mosquito larvicidal activity of isolated compounds from the rhizome of Zingiber officinale. Phyther. Res. 2008, 22, 1035-1039. [CrossRef] [PubMed]

129. Park, I.K.; Lee, S.G.; Shin, S.C.; Park, J.D.; Ahn, Y.J. Larvicidal activity of isobutylamides identified in Piper nigrum fruits against three mosquito species. J. Agric. Food Chem. 2002, 50, 1866-1870. [CrossRef] [PubMed] 
130. Park, I.K. Insecticidal activity of isobutylamides derived from Piper nigrum against adult of two mosquito species, Culex pipiens pallens and Aedes aegypti. Nat. Prod. Res. 2012, 26, 2129-2131. [CrossRef]

131. Yang, Y.C.; Lee, S.G.; Lee, H.K.; Kim, M.K.; Lee, S.H.; Lee, H.S. A Piperidine amide extracted from Piper longum L. fruit shows activity against Aedes aegypti mosquito larvae. J. Agric. Food Chem. 2002, 50, 3765-3767. [CrossRef] [PubMed]

132. Siddiqui, B.S.; Gulzar, T.; Begum, S.; Afshan, F.; Sattar, F.A. Insecticidal amides from fruits of Piper nigrum Linn. Nat. Prod. Res. 2005, 19, 143-150. [CrossRef] [PubMed]

133. Masi, M.; Cala, A.; Tabanca, N.; Cimmino, A.; Green, I.R.; Bloomquist, J.R.; Van Otterlo, W.A.L.; Macias, F.A.; Evidente, A. Alkaloids with activity against the Zika virus vector Aedes aegypti (L.)-crinsarnine and sarniensinol, two new crinine and mesembrine type alkaloids isolated from the South African plant Nerine sarniensis. Molecules 2016, 21, 1432. [CrossRef] [PubMed]

134. Ma, H.-W.; Dong, C.-Z.; Zhou, X.-M.; Bu, M.-M.; Yu, S.-Q. Aristololactam derivatives from the fruits of Aristolochia contorta Bunge. Nat. Prod. Res. 2018, 32, 2505-2509. [CrossRef]

135. Liu, X.C.; Lai, D.; Liu, Q.Z.; Zhou, L.; Liu, Q.; Liu, Z.L. Bioactivities of a new pyrrolidine alkaloid from the root barks of Orixa japonica. Molecules 2016, 21, 1665. [CrossRef]

136. Sivakumar, R.; Jebanesan, A.; Govindarajan, M.; Rajasekar, P. Larvicidal and repellent activity of tetradecanoic acid against Aedes aegypti (Linn.) and Culex quinquefasciatus (Say.) (Diptera: Culicidae). Asian Pac. J. Trop. Med. 2011, 4, 706-710. [CrossRef]

137. Sivakumar, R.; Jebanesan, A.; Govindarajan, M.; Rajasekar, P. Oviposition attractancy of dodecanoic, hexadecanoic and tetradecanoic acids against Aedes aegypti and Culex quinquefasciatus (Diptera: Culicidae). Eur. Rev. Med. Pharmacol. Sci. 2011, 15, 1172-1175.

138. Macedo, A.L.; Duprat, R.C.; Moreira, D.d.L.; Kaplan, M.A.C.; Vasconcelos, T.R.A.; Pinto, L.C.; Montenegro, R.C.; Ratcliffe, N.A.; Mello, C.B.; Valverde, A.L. Isolation of a larvicidal compound from Piper solmsianum C.DC. (Piperaceae). Nat. Prod. Res. 2017, 32, 2701-2704. [CrossRef]

139. Leite, A.C.C.F.; Kato, M.J.; Soares, R.O.A.; Guimarães, A.E.; Santos-Mallet, J.R.; Cabral, M.M.O. Grandisin caused morphological changes larval and toxicity on Aedes aegypti. Braz. J. Pharmacog. 2012, 22, 517-521. [CrossRef]

140. Maleck, M.; Dos Santos, F.C.; Serdeiro, M.; Ferreira, B.; Gunaydin, K.; De Almeida, A. Khellin: A furanochromone with toxicity against Oncopeltus fasciatus (Hemiptera) and Aedes aegypti (Diptera). J. Nat. Pharm. 2013, 4, 32-36. [CrossRef]

141. Meepagala, K.M.; Estep, A.S.; Becnel, J.J. Mosquitocidal activity of extracts from Ammi visnaga (Apiaceae) seeds. J. Agric. Chem. Environ. 2016, 5, 170-178. [CrossRef]

142. von Oppen, S.; Masuh, H.; Licastro, S.; Zerba, E.; Gonzalez-Audino, P. A Floral-derived attractant for Aedes aegypti mosquitoes. Entomol. Exp. Appl. 2015, 155, 184-192.

143. Braga, I.A.; Valle, D. Aedes aegypti: Inseticidas, mecanismos de ação e resistência. Epidemiol. Serv. Saúde 2007, 16, 279-293. [CrossRef]

144. Kostyukovsky, M.; Rafaeli, A.; Gileadi, C.; Demchenko, N.; Shaaya, E. Activation of octopaminergic receptors by essential oil constituents isolated from aromatic plants: Possible mode of action against insect pests. Pest Manag. Sci. 2002, 58, 1101-1106. [CrossRef]

145. Rattan, R.S. Mechanism of action of insecticidal secondary metabolites of plant origin. Crop Prot. 2010, 29, 913-920. [CrossRef]

146. Khanikor, B.; Parida, P.; Yadav, R.N.S.; Bora, D. Comparative mode of action of some terpene compounds against octopamine receptor and acetylcholinesterase of mosquito and human system by the help of homology modeling and docking studies. J. Appl. Pharm. Sci. 2013, 3, 6-12.

147. Pontual, E.V.; Napoleão, T.H.; Dias de Assis, C.R.; de Souza Bezerra, R.; Xavier, H.S.; Navarro, D.M.d.A.F.; Coelho, L.C.B.B.; Paiva, P.M.G. Effect of Moringa oleifera flower extract on larval trypsin and acethylcholinesterase activities in Aedes aegypti. Arch. Insect Biochem. Physiol. 2012, 79, 135-152. [CrossRef]

148. Perumalsamy, H.; Kim, J.R.; Oh, S.M.; Jung, J.W.; Ahn, Y.J.; Kwon, H.W. Novel histopathological and molecular effects of natural compound pellitorine on larval midgut epithelium and anal gills of Aedes aegypti. PLoS ONE 2013, 8, e80226. [CrossRef] [PubMed]

149. Pohlit, A.M.; Lopes, N.P.; Gama, R.A.; Tadei, W.P.; Neto, V.F. Patent literature on mosquito repellent inventions which contain plant essential oils-A review. Planta Med. 2011, 77, 598-617. [CrossRef] [PubMed] 
150. Oliveira, J.L.; Campos, E.V.R.; Fraceto, L.F. Recent developments and challenges for nanoscale formulation of botanical pesticides for use in sustainable agriculture. J. Agric. Food Chem. 2018, 66, 8898-8913. [CrossRef] [PubMed]

151. Valdiani, A.; Hansen, O.K.; Nielsen, U.B.; Johannsen, V.K.; Shariat, M.; Georgiev, M.I.; Omidvar, V.; Ebrahimi, M.; Dinanai, E.T.; Abiri, R. Bioreactor-based advances in plant tissue and cell culture: Challenges and prospects. Crit. Ver. Biotechnol. 2018, 39, 20-34. [CrossRef]

152. Liu, Y.; Chen, P.; Zhou, M.; Wang, T.; Fang, S.; Shang, X.; Fu, X. Geographic variation in the chemical composition and antioxidant properties of phenolic compounds from Cyclocarya paliurus (Batal) Iljinskaja leaves. Molecules 2018, 23, 2440. [CrossRef]

153. Zouari, N.; Ayadi, I.; Fakhfakh, N.; Rebai, A.; Zouari, S. Variation of chemical composition of essential oils in wild populations of Thymus algeriensis Boiss. et Reut., a North African endemic species. Lipids Health Dis. 2012, 11, 28. [CrossRef]

154. Al-Hamwi, M.; Bakkour, Y.; Abou-Ela, M.; El-Lakany, A.; Tabcheh, M.; El-Omar, F. Chemical composition and seasonal variation of the essential oil of Micromeria fruticosa. J. Nat. Prod. 2011, 4, 147-150.

155. Gobbo-Neto, L.; Lopes, N.P. Plantas medicinais: Fatores de influência no conteúdo de metabólitos secundários. Quim. Nova 2007, 30, 374-381. [CrossRef]

156. Workman, M.J.; Gomes, B.; Weng, J.-L.; Ista, L.K.; Jesus, C.P.; David, M.R.; Ramalho-Ortigao, M.; Genta, F.A.; Matthews, S.K.; Durvasula, R.; et al. Yeast-encapsulated essential oils: A new perspective as an environmentally friendly larvicide. Parasite Vector 2020, 13, 1-19. [CrossRef]

157. World Health Organization. Guidelines for Laboratory and Field Testing of Mosquito Larvicides. 2005. Available online: http://apps.who.int/iris/bitstream/handle/10665/69101/WHO_CDS_WHOPES_GCDPP_ 2005.13.pdf?sequence=1 (accessed on 21 February 2020).

158. Isman, M.B.; Grieneisen, M.L. Botanical insecticide research: Many publications, limited useful data. Trends Plant Sci. 2014, 19, 140-145. [CrossRef]

159. Sánchez-Bayo, F. Insecticides mode of action in relation to their toxicity to non-target organisms. J. Environ. Anal. Toxicol. 2011, S4, S4-002. [CrossRef]

160. Borrero-Landazabal, M.A.; Duque, J.E.; Mendez-Sanchez, S.C. Model to design insecticides against Aedes aegypti using in silico and in vivo analysis of different pharmacological targets. Comp. Biochem. Phys. C 2020, 229, 108664. [CrossRef] [PubMed]

161. Rueda, A.G.; Otero, A.L.C.; Duque, J.E.; Kouznetsov, V.V. Synthesis of new $\alpha$-amino nitriles with insecticidal action on Aedes aegypti (Diptera: Culicidae). Rev. Bras. Entomol. 2018, 62, 112-118. [CrossRef]

162. Devillers, J.; Lagneau, C.; Lattes, A.; Garrigues, J.C.; Clémenté, M.M.; Yébakima, A. In silico models for predicting vector control chemicals targeting Aedes aegypti. SAR QSAR Environ. Res. 2014, 25, 805-835. [CrossRef] [PubMed]

163. Dai, Y.-J.; Jia, Y.-F.; Chen, N.; Bian, W.-P.; Li, Q.-K.; Ma, Y.-B.; Chen, Y.-L.; Pei, D.-S. Zebrafish as a model system to study toxicology. Environ. Toxicol. Chem. 2014, 33, 11-17. [CrossRef] [PubMed]

164. Organisation for Economic Co-operation and Development. OECD Guidelines for the Testing of Chemicals. 2013. Available online: https://www.oecd-ilibrary.org/docserver/9789264203709-en.pdf?expires= 1591301187\&id=id\&accname=guest\&checksum=30910D5C63FC44FE9C43DDAB70F56739 (accessed on 23 March 2020).

165. Maia, J.D.; Corte, R.L..; Martinez, J.; Ubbink, J.; Prata, A.S. Improved activity of thyme essential oil (Thymus vulgaris) against Aedes aegypti larvae using a biodegradable controlled release system. Ind. Crops. Prod. 2019, 136, 110-120. [CrossRef]

166. Benelli, G.; Mehlhorn, H. Declining malaria, rising of dengue and Zika virus: Insights for mosquito vector control. Parasitol. Res. 2016, 115, 1747-1754. [CrossRef]

167. Ferreira, T.P.; Haddi, K.; Corrêa, R.F.T.; Zapata, V.L.B.; Piau, T.B.; Souza, L.F.N.; Santos, S.-M.G.; Oliveira, E.E.; Jumbo, L.O.V.; Ribeiro, B.M.; et al. Prolonged mosquitocidal activity of Siparuna guianensis essential oil encapsulated in chitosan nanoparticles. PLoS Negl. Trop. Dis. 2019, 13, e0007624. [CrossRef] [PubMed]

(C) 2020 by the authors. Licensee MDPI, Basel, Switzerland. This article is an open access article distributed under the terms and conditions of the Creative Commons Attribution (CC BY) license (http://creativecommons.org/licenses/by/4.0/). 